\title{
ON MANIN'S CONJECTURE FOR A CERTAIN SINGULAR CUBIC SURFACE
}

\author{
BY RÉGIS DE LA BRETÈCHE, TIM D. BROWNING \\ AND ULRICH DERENTHAL
}

\begin{abstract}
This paper contains a proof of the Manin conjecture for the singular cubic surface $S \subset \mathbb{P}^{3}$ that is defined by the equation $x_{1} x_{2}^{2}+x_{2} x_{0}^{2}+x_{3}^{3}=0$. In fact if $U \subset S$ is the Zariski open subset obtained by deleting the unique line from $S$, and $H$ is the usual exponential height on $\mathbb{P}^{3}(\mathbb{Q})$, then the height zeta function $\sum_{x \in U(\mathbb{Q})} H(x)^{-s}$ is analytically continued to the half-plane $\Re e(s)>9 / 10$.

(C) 2007 Elsevier Masson SAS
\end{abstract}

RÉSUMÉ. - Cet article contient une preuve de la conjecture de Manin pour la surface cubique singulière $S \subset \mathbb{P}^{3}$ définie par $x_{1} x_{2}^{2}+x_{2} x_{0}^{2}+x_{3}^{3}=0$. En effet, si $U \subset S$ est l'ouvert obtenu en enlevant l'unique droite contenue dans $S$ et $H$ est la fonction des hauteurs usuelle de $\mathbb{P}^{3}(\mathbb{Q})$, alors la fonction zêta des hauteurs $\sum_{x \in U(\mathbb{Q})} H(x)^{-s}$ peut être prolongée de manière analytique au demi-plan $\Re e(s)>9 / 10$.

(c) 2007 Elsevier Masson SAS

\section{Introduction}

Let $S \subset \mathbb{P}^{3}$ be a cubic surface that is defined over $\mathbb{Q}$ and has isolated singularities. As soon as $S$ contains a single $\mathbb{Q}$-rational point the set of rational points $S(\mathbb{Q})=S \cap \mathbb{P}^{3}(\mathbb{Q})$ is dense in the Zariski topology, and it is natural to seek a finer interpretation of this density. Given a point $x=\left[x_{0}, \ldots, x_{3}\right] \in \mathbb{P}^{3}(\mathbb{Q})$, with $x_{0}, \ldots, x_{3} \in \mathbb{Z}$ such that $\operatorname{gcd}\left(x_{0}, \ldots, x_{3}\right)=1$, we let

$$
H(x)=\max \left\{\left|x_{0}\right|,\left|x_{1}\right|,\left|x_{2}\right|,\left|x_{3}\right|\right\} .
$$

Then $H: \mathbb{P}^{3}(\mathbb{Q}) \rightarrow \mathbb{R}_{\geqslant 0}$ is the exponential height attached to the anticanonical embedding of $S$, metrized by the choice of norm $|\mathbf{z}|:=\max _{0 \leqslant i \leqslant 3}\left|z_{i}\right|$ on $\mathbb{R}^{4}$. We may define the quantity

$$
N_{U, H}(B)=\#\{x \in U(\mathbb{Q}): H(x) \leqslant B\},
$$

for any $B \geqslant 1$ and any Zariski open subset $U \subseteq S$. If $S$ contains lines defined over $\mathbb{Q}$ then $N_{S, H}(B)$ will be dominated by the rational points of height at most $B$ that lie on such lines. For this reason one is most interested in studying the counting function $N_{U, H}(B)$ for the open subset $U \subset S$ obtained by deleting all of the lines from $S$.

In this setting Manin [9] has formulated a far-reaching conjecture for the asymptotic behaviour of $N_{U, H}(B)$, as $B \rightarrow \infty$. This states that there is a non-negative constant $c_{S, H}$ and a positive integer $\rho$ such that

$$
N_{U, H}(B)=c_{S, H} B(\log B)^{\rho-1}(1+\mathrm{o}(1)),
$$

ANNALES SCIENTIFIQUES DE L'ÉCOLE NORMALE SUPÉRIEURE 0012-9593/01/@ 2007 Elsevier Masson SAS. All rights reserved. 
as $B \rightarrow \infty$. Here $\rho$ is conjectured to be the rank of the Picard group of the minimal desingularisation of $S$, and the constant $c_{S, H}$ has also been given a conjectural interpretation at the hands of Peyre [15], Batyrev and Tschinkel [1], and Salberger [16].

Although Manin's conjecture can actually be applied to a rather general class of algebraic variety, in which context it has met with a reasonable degree of success, the situation for cubic surfaces is rather less satisfactory. For non-singular cubic surfaces the best result that we have is the upper bound $N_{U, H}(B)=\mathrm{O}_{\varepsilon, S}\left(B^{4 / 3+\varepsilon}\right)$, which is due to Heath-Brown [11] and applies when the surface contains three coplanar lines defined over $\mathbb{Q}$. For singular cubic surfaces better estimates are available. A modern classification of such surfaces can be found in the work of Bruce and Wall [6], which shows in particular that there are only finitely many classes to consider over $\overline{\mathbb{Q}}$, these being essentially classified by their singularity type. The Manin conjecture for singular cubic surfaces has only been settled in particularly simple cases, such as the singular toric variety

$$
x_{0}^{3}=x_{1} x_{2} x_{3}
$$

of singularity type $3 \mathbf{A}_{2}$. Several authors have studied this surface, and the sharpest estimate available is that due to the first author [2]. Further work worth mentioning is that due to Chambert-Loir and Tschinkel [7], who have established Manin's conjecture for any cubic surface arising as an equivariant compactification of $\mathbb{G}_{a}^{2}$. There is also the work of Heath-Brown [12] and the second author [5]. These latter results provide upper and lower bounds of the expected order of magnitude for the counting function associated to two singular cubic surfaces: the Cayley cubic surface

$$
\frac{1}{x_{0}}+\frac{1}{x_{1}}+\frac{1}{x_{2}}+\frac{1}{x_{3}}=0
$$

of singularity type $4 \mathbf{A}_{1}$, and the surface

$$
x_{1} x_{2} x_{3}=x_{0}\left(x_{1}+x_{2}+x_{3}\right)^{2}
$$

containing a $\mathbf{D}_{4}$-singularity, respectively.

We are now ready to reveal the contribution that we have been able to make to this topic. Thus the primary goal of this paper is to verify the Manin conjecture for the cubic surface

$$
x_{1} x_{2}^{2}+x_{2} x_{0}^{2}+x_{3}^{3}=0
$$

which we henceforth denote by $S$. This surface contains a unique singularity of type $\mathbf{E}_{6}$, and a unique line $\ell$ which is given by $x_{2}=x_{3}=0$. It has been shown by Hassett and Tschinkel [10, Remark 4.3] that $S$ is not an equivariant compactification of $\mathbb{G}_{a}^{2}$, so that it is not covered by [7]. Let $U \subset S$ be the open subset formed by deleting $\ell$ from $S$. Then we have the following result.

THEOREM 1. - Let $\varepsilon>0$. Then there exists a polynomial $P$ of degree 6 such that

$$
N_{U, H}(B)=B P(\log B)+\mathrm{O}_{\varepsilon}\left(B^{10 / 11+\varepsilon}\right),
$$

for any $B \geqslant 1$. Moreover the leading coefficient of $P$ is equal to

$$
\frac{\omega_{\infty}}{6220800} \prod_{p}\left(1-\frac{1}{p}\right)^{7}\left(1+\frac{7}{p}+\frac{1}{p^{2}}\right),
$$

$4^{e}$ SÉRIE - TOME $40-2007-N^{\circ} 1$ 
where

$$
\omega_{\infty}=12 \underset{\left\{(t, u, v) \in \mathbb{R}^{3}:\left|t^{2}+u^{3}\right| \leqslant 1,0 \leqslant t v^{3} \leqslant 1,0 \leqslant v \leqslant 1,\left|u v^{4}\right| \leqslant 1\right\}}{\int} \mathrm{d} t \mathrm{~d} u \mathrm{~d} v .
$$

We shall verify in $\$ 2$ that Theorem 1 is in agreement with the Manin conjecture. In the classification of singular cubic surfaces over $\overline{\mathbb{Q}}, S$ is the only cubic surface with an $\mathbf{E}_{6}$ singularity, up to projectivity [6]. In fact this is the most extreme type of singularity that a cubic surface can possess. Given that non-singular cubic surfaces seem so difficult to tackle, our success with (1.2) perhaps reflects the fact that we are as far away from the non-singular setting as possible.

It is now well-recognised that universal torsors play a central rôle in proofs of the Manin conjecture for Fano varieties. There is no exception to this philosophy in the present work. Thus in the proof of Theorem 1 crucial use is made of the universal torsor above the minimal desingularisation $\widetilde{S}$ of $S$, which turns out to have the natural affine embedding

$$
\tau_{\ell} \xi_{\ell}^{3} \xi_{4}^{2} \xi_{5}+\tau_{2}^{2} \xi_{2}+\tau_{1}^{3} \xi_{1}^{2} \xi_{3}=0
$$

This has been calculated by Hassett and Tschinkel using the Cox ring [10]. However in §7 we shall establish a completely explicit bijection between $U(\mathbb{Q})$ and a suitable set of integral points satisfying this equation, via an elementary analysis of the equation defining $S$. It will become apparent that the passage to the universal torsor is really only the first step on the road to proving Theorem 1, and that a considerable amount of input is still required.

Once the passage to the universal torsor is accomplished, the proof of Theorem 1 broadly follows the strategy of the first two authors [3,4], where key use is made of the fact that the torsor equation in each case has precisely three terms. In counting integral solutions to (1.4), subject to certain constraints, we shall begin by fixing most of the variables and summing only over the variables $\tau_{1}, \tau_{2}, \tau_{\ell}$. The key idea is then to view the equation as a congruence

$$
\tau_{2}^{2} \xi_{2} \equiv-\tau_{1}^{3} \xi_{1}^{2} \xi_{3}\left(\bmod \xi_{\ell}^{3} \xi_{4}^{2} \xi_{5}\right),
$$

in order to take care of the summation over $\tau_{\ell}$. One proceeds to employ standard facts about the number of integer solutions to polynomial congruences that are restricted to lie in certain regions. This produces a main term and an error term, and the rest of the proof involves summing each of these terms over all of the remaining variables. While the treatment of the main term is relatively routine, the treatment of the error term presents a much more serious obstacle. There are two main ingredients in this part of the work, both of which are rooted in the theory of exponential sums. The first involves showing that sequences of the form $\left(a x^{3}+b x^{2}\right) / q$ are equidistributed modulo 1 as $x$ ranges over the ring $\mathbb{Z} / q \mathbb{Z}$, for fixed integers $a, b, q$ such that $\operatorname{gcd}(a, b, q)=1$, and the second constitutes a delicate analysis of certain exponential sums involving real-valued functions that arise in our work. Whereas the first ingredient is independent of the choice of norm used to metrize the height function $H$, and so may be thought of as purely "arithmetic", the second ingredient is intimately connected to the norm selected and may be thought of as being "analytic" in nature.

Given the shape of the estimate in Theorem 1 it is no surprise that we are able to say something about the corresponding height zeta function. As above let $U \subset S$ be the open subset of the surface (1.2) that is formed by deleting the unique line from it. Then we may define

$$
Z_{U, H}(s):=\sum_{x \in U(\mathbb{Q})} \frac{1}{H(x)^{s}},
$$


for $\Re e(s)>1$, and Theorem 1 can be used to show that $Z_{U, H}(s)$ has a meromorphic continuation to the half-plane $\Re e(s)>10 / 11$. In fact by returning to the proof of Theorem 1 we are able to say something about the analytic structure of $Z_{U, H}(s)$ to the left of the line $\Re e(s)=10 / 11$. For $\Re e(s)>0$ we define the functions

$$
\begin{aligned}
& E_{1}(s+1):=\zeta(2 s+1) \zeta(3 s+1)^{2} \zeta(4 s+1)^{2} \zeta(5 s+1) \zeta(6 s+1), \\
& E_{2}(s+1):=\frac{\zeta(13 s+3)^{5} \zeta(14 s+3)^{2}}{\zeta(7 s+2)^{4} \zeta(8 s+2)^{4} \zeta(9 s+2)^{2} \zeta(10 s+2) \zeta(19 s+4)^{2}} .
\end{aligned}
$$

It is easily seen that $E_{1}(s)$ has a meromorphic continuation to the entire complex plane with a single pole at $s=1$, and similarly, $E_{2}(s)$ is holomorphic and bounded on the half-plane $\Re e(s)>9 / 10$. We are now ready to record precisely what we have been able to say about the height zeta function.

THEOREM 2. - Let $\varepsilon>0$. Then there exist a constant $\beta \in \mathbb{R}$, and functions $G_{1}(s), G_{2}(s)$ that are holomorphic on the half-plane $\Re e(s) \geqslant 43 / 48+\varepsilon$, such that for $\Re e(s)>1$ we have

$$
Z_{U, H}(s)=E_{1}(s) E_{2}(s) G_{1}(s)+\frac{12 / \pi^{2}+2 \beta}{s-1}+G_{2}(s) .
$$

In particular $(s-1)^{7} Z_{U, H}(s)$ has a holomorphic continuation to the half-plane $\Re e(s)>9 / 10$.

Explicit expressions for $\beta, G_{1}$ and $G_{2}$ can be found in (8.50), (10.3) and (10.1), respectively. It can be seen there that $G_{1}(s)$ is actually holomorphic and bounded on the half-plane $\Re e(s) \geqslant$ $5 / 6+\varepsilon$, and that

$$
G_{2}(s) \ll 1+|\Im m(s)|
$$

for $\Re e(s) \geqslant 43 / 48+\varepsilon$.

With more work it is likely that the constant $43 / 48$ can be reduced slightly, although all we need to deduce the final sentence in Theorem 2 is the fact that $43 / 48<9 / 10$. However, under the assumption of the Riemann hypothesis it is clear that $E_{2}(s)$ is holomorphic for $\Re e(s)>8 / 9$, whence $Z_{U, H}(s)$ has a meromorphic continuation to the half-plane $\Re e(s)>43 / 48$.

Theorem 2 bears a striking resemblance to the results obtained by the first two authors [3,4], in their work on the Manin conjecture for singular del Pezzo surfaces of degree 4, which also contain explicit expressions for the corresponding height zeta functions. Thus in addition to the "main term" $E_{1}(s) E_{2}(s) G_{1}(s)$, all of these results have a term $\frac{12}{\pi^{2}}(s-1)^{-1}$ that corresponds here to the residual conic obtained by intersecting $S$ with the plane $x_{3}=0$, and a further " $\beta$-term". In Theorem 2 the constant $\beta$ has much in common with the corresponding result in [3], arising as it does through the application of results about the equidistribution of squares in a fixed residue class. However the argument needed here is distinctly subtler than anything previously encountered.

The genesis of this paper lies in an earlier paper due to the third author [8], who succeeded in proving a version of Theorem 1 with an error term of $\mathrm{O}\left(B(\log B)^{2}\right)$. The main contribution of the first and second authors has therefore been to push the analysis further, to the extent that we now have results of the precision detailed above. During the final preparation of this paper, the authors have been made aware of the doctoral thesis of M. Joyce at Brown University, who has independently established the Manin conjecture for the $\mathbf{E}_{6}$ cubic surface $S$. His main result is weaker than that obtained in our paper, since he only establishes an asymptotic formula with an error term of $\mathrm{O}\left(B(\log B)^{5}\right)$.

We end this introduction by giving an overview of the contents of this paper. As indicated above, we shall begin in $\$ 2$ by showing that Theorem 1 is in complete agreement with the

$4^{e}$ SÉRIE - TOME $40-2007-\mathrm{N}^{\circ} 1$ 
Manin conjecture. Next in $\S 3$ and $\S 4$ we shall collect together most of the material concerning exponential sums and equidistribution that will be crucial for our treatment of the error terms discussed above. In $\$ 5$ we shall introduce and analyse a number of real-valued functions that will arise in our work, before turning in $\S 6$ to a preliminary estimate for the counting function $N_{U, H}(B)$. The passage to the universal torsor will take place in $\S 7$, and the conclusion of the proof of Theorem 1 will form the contents of $\S 8$ and $\$ 9$. Finally we shall deduce the statement of Theorem 2 in $\S 10$.

\section{Conformity with the Manin conjecture}

In this section we shall review some of the geometry of the surface $S \subset \mathbb{P}^{3}$, with a view to calculating the invariants appearing in Manin's conjecture and its refinement by Peyre. Let $\widetilde{S}$ denote the minimal desingularisation of $S$, and let $\pi: \widetilde{S} \rightarrow S$ denote the corresponding blow-up map. We let $F_{1}, \ldots, F_{6}$ denote the exceptional divisors of $\pi$. Then the divisors $F_{1}, \ldots, F_{6}$ are all defined over $\mathbb{Q}$, and together with the line $\ell$, they generate the Picard group $\operatorname{Pic}(\widetilde{S})$ of $\widetilde{S}$. In particular we have $\rho=7$ in (1.1).

Turning to the conjectured value of the constant $c_{S, H}$ in (1.1), we follow the notation and methodology of Peyre [15]. With this in mind we proceed by establishing the following result.

LEMMA 1. - We have $c_{S, H}=\alpha(\widetilde{S}) \beta(\widetilde{S}) \omega_{H}(\widetilde{S})$, with

$$
\alpha(\widetilde{S})=\frac{1}{6220800}, \quad \beta(\widetilde{S})=1, \quad \omega_{H}(\widetilde{S})=\omega_{\infty} \prod_{p}\left(1-\frac{1}{p}\right)^{7}\left(1+\frac{7}{p}+\frac{1}{p^{2}}\right),
$$

where $\omega_{\infty}$ is given by $(1.3)$.

Proof. - We have already observed that $\left\{F_{1}, F_{2}, F_{3}, \ell, F_{4}, F_{5}, F_{6}\right\}$ is a basis for $\operatorname{Pic}(\widetilde{S})$. It follows from [10] that the effective cone $\Lambda_{\text {eff }}(\widetilde{S})$ is generated by the elements of this basis, and that the dual cone of nef divisors is simplical, in the sense that it is generated by $\rho=7$ elements. Moreover the anticanonical divisor $-K_{\widetilde{S}}$ of $\widetilde{S}$ is given by

$$
-K_{\widetilde{S}}=2 F_{1}+3 F_{2}+4 F_{3}+3 \ell+4 F_{4}+5 F_{5}+6 F_{6} .
$$

We may therefore write $-K_{\widetilde{S}}=\boldsymbol{\lambda}$ in the basis $\left\{F_{1}, F_{2}, F_{3}, \ell, F_{4}, F_{5}, F_{6}\right\}$, with

$$
\boldsymbol{\lambda}=\left(\lambda_{1}, \lambda_{2}, \lambda_{3}, \lambda_{\ell}, \lambda_{4}, \lambda_{5}, \lambda_{6}\right):=(2,3,4,3,4,5,6) .
$$

Thus the definition of $\alpha(\widetilde{S})$ reveals that

$$
\alpha(\widetilde{S})=\operatorname{meas}\left\{\mathbf{t} \in \mathbb{R}_{\geqslant 0}^{7}: \boldsymbol{\lambda} . \mathbf{t}=1\right\}=\frac{1}{6 ! \lambda_{1} \lambda_{2} \lambda_{3} \lambda_{\ell} \lambda_{4} \lambda_{5} \lambda_{6}}=\frac{1}{6220800},
$$

where we have written $\mathbf{t}=\left(t_{1}, t_{2}, t_{3}, t_{\ell}, t_{4}, t_{5}, t_{6}\right)$. Next we note that

$$
\beta(\widetilde{S}):=\# H^{1}\left(\operatorname{Gal}(\overline{\mathbb{Q}} / \mathbb{Q}), \operatorname{Pic}(\widetilde{S}) \otimes_{\mathbb{Q}} \overline{\mathbb{Q}}\right)=1,
$$

since $S$ is split over $\mathbb{Q}$. Finally we must consider the factor $\omega_{H}(\widetilde{S})$, which corresponds to a product of local densities. According to the definition of $\omega_{H}(\widetilde{S})$ we have 


$$
\begin{aligned}
\omega_{H}(\widetilde{S}) & :=\lim _{s \rightarrow 1}\left((s-1)^{\mathrm{rk} \operatorname{Pic}(\widetilde{S})} L(s, \operatorname{Pic}(\widetilde{S}))\right) \omega_{\infty} \prod_{p} \frac{\omega_{p}}{L_{p}(1, \operatorname{Pic}(\widetilde{S}))} \\
& =\omega_{\infty} \prod_{p}\left(1-\frac{1}{p}\right)^{7} \omega_{p},
\end{aligned}
$$

since $L(s, \operatorname{Pic}(\widetilde{S}))=\zeta(s)^{7}$, in our case. The calculation of $\omega_{p}$ is straightforward, and ultimately leads to the conclusion that

$$
\omega_{p}=1+\frac{7}{p}+\frac{1}{p^{2}} .
$$

To compute $\omega_{\infty}$ we parametrise the points by writing $x_{1}$ as a function of $x_{0}, x_{2}, x_{3}$ in $f(\mathbf{x})=$ $x_{1} x_{2}^{2}+x_{2} x_{0}^{2}+x_{3}^{3}$. Since $\mathbf{x}=-\mathbf{x}$ in $\mathbb{P}^{3}$, we may assume $x_{2} \geqslant 0$. On observing that $\frac{\partial f}{\partial x_{1}}=x_{2}^{2}$, the Leray form $\omega_{L}(\widetilde{S})$ is given by $x_{2}^{-2} \mathrm{~d} x_{0} \mathrm{~d} x_{2} \mathrm{~d} x_{3}$, and so

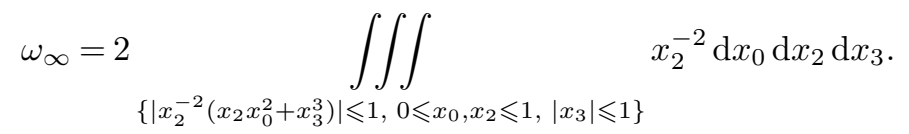

But then the change of variables $x_{0}=t x_{2}^{1 / 2}, x_{3}=u x_{2}^{2 / 3}$ and $x_{2}=v^{6}$, easily yields the value of $\omega_{\infty}$ given in (1.3). This completes the proof of the lemma.

On combining Lemma 1 with our earlier observation that $\rho=7$ in (1.1), we therefore conclude that Theorem 1 is in accordance with the Manin conjecture.

\section{Exponential sums}

During the course of the subsequent section we shall need good upper bounds for the modulus of several exponential sums. We have collected together the results that we shall need in the present section, throughout which we employ the usual notation $e(x)=e^{2 \pi i x}$ and $e_{q}(x)=e(x / q)$, for any $q \in \mathbb{N}$ and $x \in \mathbb{R}$, and always take $\mathbb{N}$ to denote the set of positive integers. Furthermore, we shall write $\lfloor x\rfloor$ (resp. $\lceil x\rceil$ ) for the integer part (resp. the ceiling) of any $x \in \mathbb{R}$.

Let $a, b \in \mathbb{Z}$ and let $q \in \mathbb{N}$. The primary goal of this section is then to estimate the cubic exponential sum

$$
S_{q}(a, b):=\sum_{\substack{x=1 \\ \operatorname{gcd}(x, q)=1}}^{q} e_{q}\left(a x^{3}+b x^{2}\right),
$$

under the assumption that $\operatorname{gcd}(a, b, q)=1$. Our approach will involve relating $S_{q}(a, b)$ to the complete exponential sum

$$
T_{q}(a, b):=\sum_{x=1}^{q} e_{q}\left(a x^{3}+b x^{2}\right) .
$$

We begin by recording the multiplicativity properties

$$
\begin{aligned}
& S_{u v}(a, b)=S_{u}\left(v^{2} a, v b\right) S_{v}\left(u^{2} a, u b\right), \\
& T_{u v}(a, b)=T_{u}\left(v^{2} a, v b\right) T_{v}\left(u^{2} a, u b\right),
\end{aligned}
$$

$4^{e}$ SÉRIE - TOME $40-2007-N^{\circ} 1$ 
that are valid for any coprime $u, v \in \mathbb{N}$ such that $\operatorname{gcd}(a, b, u v)=1$. These equalities follow from the Chinese remainder theorem (see [20, Lemma 2.10], for example). We are now ready to estimate (3.1) in the case $b=0$.

LEMmA 2. - Let $\varepsilon>0$ and suppose that $\operatorname{gcd}(a, q)=1$. Then we have

$$
S_{q}(a, 0) \ll_{\varepsilon} q^{2 / 3+\varepsilon} .
$$

Proof. - In view of (3.3) and the estimate $A^{\omega(q)}=\mathrm{O}_{A, \varepsilon}\left(q^{\varepsilon}\right)$, it will suffice to show that $S_{p^{\ell}}(a, 0) \ll p^{2 \ell / 3}$, for any prime $p$ such that $p \nmid a$, and any $\ell \in \mathbb{N}$. But when $\ell \geqslant 3$ it follows that

$$
S_{p^{\ell}}(a, 0)=T_{p^{\ell}}(a, 0)-p^{2} T_{p^{\ell-3}}(a, 0),
$$

whence [20, Eq. (7.9)] yields

$$
S_{p^{\ell}}(a, 0) \ll p^{2 \ell / 3}+p^{2} p^{2(\ell-3) / 3} \ll p^{2 \ell / 3},
$$

when $\ell \geqslant 3$. The same sort of calculation suffices to handle the cases $\ell=1$ and $\ell=2$, which therefore completes the proof of the lemma.

We now turn to the task of estimating (3.1) for non-zero values of $b$, for which we shall need a corresponding estimate for (3.2) in the case that $b$ is non-zero. This is provided for us by the following result.

LEMmA 3. - Let $p$ be a prime such that $\operatorname{gcd}(a, b, p)=1$ and let $\ell \in \mathbb{N}$. Then we have

$$
\left|T_{p^{\ell}}(a, b)\right| \leqslant 2 p^{\ell / 2} \operatorname{gcd}\left(b, p^{\ell}\right) .
$$

Proof. - The case in which $\ell=1$ is handled by the well-known estimate of Weil [21], which gives $\left|T_{p}(a, b)\right| \leqslant 2 p^{1 / 2}$. The case in which $\ell \geqslant 2$ follows from the work of Loxton and Vaughan [14, Theorem 1]. This completes the proof of Lemma 3.

We are now ready to record an estimate for (3.1) that is valid for any choice of $a, b \in \mathbb{Z}$ and $q \in \mathbb{N}$ such that $\operatorname{gcd}(a, b, q)=1$.

LEMMA 4. - Let $\varepsilon>0$ and suppose that $\operatorname{gcd}(a, b, q)=1$. Then we have

$$
S_{q}(a, b) \ll_{\varepsilon} q^{1 / 2+\varepsilon} \operatorname{gcd}(b, q) .
$$

Proof. - As in the proof of Lemma 2, the properties in (3.3) render it sufficient to establish the bound $S_{p^{\ell}}(a, b) \ll p^{\ell / 2} \operatorname{gcd}\left(b, p^{\ell}\right)$, for any prime $p$ such that $p \nmid \operatorname{gcd}(a, b)$, and any $\ell \in \mathbb{N}$. When $\ell \geqslant 2$ it follows that

$$
S_{p^{\ell}}(a, b)=T_{p^{\ell}}(a, b)-p T_{p^{\ell-2}}(a p, b),
$$

whence Lemma 3 yields $S_{p^{\ell}}(a, b) \ll p^{\ell / 2}$, if $\ell \geqslant 2$ and $p \nmid b$. If $p \mid b$, then we may write $b=p b^{\prime}$. In this case Lemma 3 yields

$$
\begin{aligned}
S_{p^{\ell}}(a, b) & =T_{p^{\ell}}(a, b)-p^{2} T_{p^{\ell-3}}\left(a, b^{\prime}\right) \\
& \ll p^{\ell / 2} \operatorname{gcd}\left(b, p^{\ell}\right)+p^{2+(\ell-3) / 2} \operatorname{gcd}\left(b^{\prime}, p^{\ell-3}\right) \\
& \ll p^{\ell / 2} \operatorname{gcd}\left(b, p^{\ell}\right),
\end{aligned}
$$


if $\ell \geqslant 3$. Together these two estimates handle the case in which $\ell \geqslant 3$. Finally, the same sort of calculation suffices to handle the cases $\ell=1$ and $\ell=2$, which therefore completes the proof of Lemma 4.

Now let $I=\left[t_{1}, t_{2}\right] \subset \mathbb{R}$ be any closed interval, and let $f$ be a real-valued function on it. Then for given $a, b, q \in \mathbb{Z}$ such that $q>0$, the remainder of this section is concerned with the size of the exponential sum

$$
A_{I}(q ; a, b, f):=\sum_{t_{1}<n \leqslant t_{2}} e_{q}(a n+b f(n)) .
$$

In particular we shall want to obtain a saving over the trivial upper bound

$$
\left|A_{I}(q ; a, b, f)\right| \leqslant t_{2}-t_{1}+1
$$

by restricting our attention to suitable families of real-valued functions. For an interval $I=$ $\left[t_{1}, t_{2}\right] \subset \mathbb{R}$ and a real number $\lambda_{0} \geqslant 1$, we shall say that a real-valued function $f$ belongs to the set $C^{1}\left(I ; \lambda_{0}\right)=C^{1}\left(t_{1}, t_{2} ; \lambda_{0}\right)$ if $f$ is differentiable on $I$, with

$$
\left|f\left(t_{2}\right)-f\left(t_{1}\right)\right|+1 \leqslant \lambda_{0},
$$

and if $f^{\prime}$ is monotonic and of constant sign on $\left(t_{1}, t_{2}\right)$. We then have the following result.

Lemma 5. - Let $I \subset \mathbb{R}$ be any closed interval and let $\lambda_{0} \geqslant 1$. Suppose that $a, b, q \in \mathbb{Z}$ such that $0<|a| \leqslant q / 2$, and let $f \in C^{1}\left(I ; \lambda_{0}\right)$. Then we have

$$
A_{I}(q ; a, b, f) \ll \frac{1}{|a|}\left(q+|b| \lambda_{0}\right) .
$$

Proof. - Suppose that $I=\left[t_{1}, t_{2}\right]$, for $t_{1}<t_{2}$. To establish the lemma, we write $A_{t}(q ; a)$ for the linear exponential sum $A_{\left[t_{1}, t\right]}(q ; a, 0,0)$ for $t \in\left(t_{1}, t_{2}\right]$. Then

$$
A_{t}(q ; a)=\frac{e_{q}\left(a\left\lceil t_{1}\right\rceil\right)-e_{q}(a(\lfloor t\rfloor+1))}{1-e(a / q)},
$$

whence

$$
A_{t}(q ; a) \ll \frac{1}{|1-e(a / q)|}=\frac{1}{|\sin (\pi a / q)|} \ll \frac{q}{|a|},
$$

since $|a| \leqslant q / 2$. Set $F(t)=e_{q}(b f(t))$ for $t_{1}<t \leqslant t_{2}$, and $F(t)=0$ otherwise. Then in view of (3.7) and (3.8), a simple application of partial summation yields

$$
\begin{aligned}
A_{I}(q ; a, b, f) & =A_{t_{2}}(q ; a) F\left(t_{2}\right)-\int_{t_{1}}^{t_{2}} A_{t}(q ; a) F^{\prime}(t) \mathrm{d} t \\
& =-\int_{t_{1}}^{t_{2}} A_{t}(q ; a) F^{\prime}(t) \mathrm{d} t+\mathrm{O}\left(|a|^{-1} q\right) \\
& =\int_{t_{1}}^{t_{2}} \frac{e_{q}(a(\lfloor t\rfloor+1))}{1-e(a / q)} F^{\prime}(t) \mathrm{d} t+\mathrm{O}\left(|a|^{-1} q\right) .
\end{aligned}
$$

$4^{\text {e }}$ SÉRIE - TOME $40-2007-\mathrm{N}^{\circ} 1$ 
But then the lemma easily follows from the observation that

$$
\int_{t_{1}}^{t_{2}}\left|F^{\prime}(t)\right| \mathrm{d} t \leqslant \frac{2 \pi|b|}{q} \int_{t_{1}}^{t_{2}}\left|f^{\prime}(t) F(t)\right| \mathrm{d} t,
$$

this latter integral being $\mathrm{O}\left(\lambda_{0}\right)$.

We can do somewhat better by further restricting the class of functions $f$ under consideration. Let $I \subset \mathbb{R}$ be a closed interval, and let $j, \lambda_{0}, \lambda_{1}, \lambda_{2} \in \mathbb{R}$ such that

$$
j, \lambda_{0}, \lambda_{1} \geqslant 1, \quad \lambda_{2}>0 .
$$

We say that a real-valued function $f$ belongs to the set $C^{2}\left(I ; \lambda_{0}, \lambda_{1}, \lambda_{2}, j\right)$ if $f$ is twice differentiable on $I$, with $f \in C^{1}\left(I ; \lambda_{0}\right)$ and

$$
\left|f^{\prime}(t)\right| \leqslant \lambda_{1}, \quad \lambda_{2} \leqslant\left|f^{\prime \prime}(t)\right| \leqslant j \lambda_{2},
$$

throughout $I$. On defining the notation

$$
\mathfrak{m}(I):=\operatorname{meas}(I)+1
$$

we then have the following result.

LEMma 6. - Let $I \subset \mathbb{R}$ be any closed interval and let $j, \lambda_{0}, \lambda_{1}, \lambda_{2} \in \mathbb{R}$ such that (3.10) holds. Suppose that $a, b, q \in \mathbb{Z}$ such that $0<|a| \leqslant q / 2$, and let $f \in C^{2}\left(I ; \lambda_{0}, \lambda_{1}, \lambda_{2}, j\right)$. Then we have

$$
A_{I}(q ; a, b, f) \ll \frac{1}{|a|}\left(q+\lambda_{1} E\right)
$$

where

$$
E=\frac{|b|^{1 / 2} q^{1 / 2}}{\lambda_{2}^{1 / 2}}+\frac{|b|^{3 / 2} j \lambda_{2}^{1 / 2} \mathfrak{m}(I)}{q^{1 / 2}}+\frac{b^{2} \lambda_{0}}{q} .
$$

Proof. - Suppose that $I=\left[t_{1}, t_{2}\right]$, for $t_{1}<t_{2}$. We begin by following the proof of Lemma 5 . Thus we may assume that (3.9) holds, with $|1-e(a / q)|^{-1} \ll|a|^{-1} q$ and $F(t)=e_{q}(b f(t))$ for $t_{1}<t \leqslant t_{2}$. Then it is not hard to conclude that

$$
A_{I}(q ; a, b, f) \ll \frac{q}{|a|}(1+|J|),
$$

where

$$
\begin{aligned}
J & =\sum_{t_{1}<n \leqslant t_{2}} e_{q}(a n)(F(n)-F(n-1)) \\
& =\sum_{t_{1}<n \leqslant t_{2}} e_{q}(a n+b f(n))\left(1-e_{q}(b(f(n-1)-f(n)))\right) .
\end{aligned}
$$

Let $n \in\left(t_{1}, t_{2}\right]$. There exists $\xi \in(n-1, n)$ such that $f(n)-f(n-1)=f^{\prime}(\xi)$, by the mean value theorem. Since $f \in C^{2}\left(I ; \lambda_{0}, \lambda_{1}, \lambda_{2}, j\right)$, it follows that

$$
\sup _{t_{1}<n \leqslant t_{2}}|f(n)-f(n-1)| \leqslant \lambda_{1} .
$$

ANNALES SCIENTIFIQUES DE L'ÉCOLE NORMALE SUPÉRIEURE 
In view of the familiar estimate $e^{i t}=1+i t+\mathrm{O}\left(t^{2}\right)$, that is valid for any $t \in \mathbb{R}$, we deduce that

$$
\begin{aligned}
1-e_{q}(b(f(n-1)-f(n)))= & 2 \pi i b(f(n)-f(n-1)) / q \\
& +\mathrm{O}\left(b^{2} \lambda_{1}|f(n)-f(n-1)| / q^{2}\right) .
\end{aligned}
$$

Hence

$$
J \ll \frac{|b|}{q}|S|+\frac{b^{2} \lambda_{1}}{q^{2}} \sum_{t_{1}<n \leqslant t_{2}}|f(n)-f(n-1)| \ll \frac{|b|}{q}|S|+\frac{b^{2} \lambda_{0} \lambda_{1}}{q^{2}},
$$

where

$$
S=\sum_{t_{1}<n \leqslant t_{2}} e_{q}(a n+b f(n))(f(n)-f(n-1)) .
$$

Our final task is to handle this sum.

Let $G(t)=f(t)-f(t-1)$ and

$$
T_{t}=\sum_{t_{1}<n \leqslant t} e_{q}(a n+b f(n))
$$

for any $t \in\left(t_{1}, t_{2}\right]$. Then the second derivative estimate of Van der Corput [18, Theorem 5.9] yields

$$
T_{t} \ll j \mathfrak{m}(I)\left(|b| \lambda_{2} / q\right)^{1 / 2}+\left(|b| \lambda_{2} / q\right)^{-1 / 2},
$$

since $f \in C^{2}\left(I ; \lambda_{0}, \lambda_{1}, \lambda_{2}, j\right)$ and $t \leqslant t_{2}$. Now an application of partial summation gives

$$
S=T_{t_{2}} G\left(t_{2}\right)-\int_{t_{1}}^{t_{2}} T_{t} G^{\prime}(t) \mathrm{d} t
$$

On applying the mean value theorem to $G$ and $G^{\prime}$, we therefore conclude from (3.14) that

$$
S \ll\left(j \mathfrak{m}(I)\left(|b| \lambda_{2} / q\right)^{1 / 2}+\left(|b| \lambda_{2} / q\right)^{-1 / 2}\right)\left(\lambda_{1}+\int_{t_{1}}^{t_{2}}\left|G^{\prime}(t)\right| \mathrm{d} t\right) .
$$

But the last integral here is clearly $\mathrm{O}\left(\lambda_{1}\right)$, since $f^{\prime}$ is monotonic and of constant sign on $\left(t_{1}, t_{2}\right)$. Putting all of this together we therefore conclude that (3.13) holds, with

$$
q J \ll|b||S|+\frac{b^{2} \lambda_{0} \lambda_{1}}{q} \ll \lambda_{1} E,
$$

in the notation of (3.12). This completes the proof of the lemma.

\section{Equidistribution}

During the course of the proof of Theorem 1, as carried out in $\S \S 6-10$ below, we shall need a precise expression for the number of integers in an interval that lie in a fixed congruence class. Define the real-valued function $\psi(t)=\{t\}-1 / 2$, where $\{t\}$ denotes the fractional part of $t \in \mathbb{R}$. Then $\psi$ is periodic with period 1 , and we have the following simple estimate [3, Lemma 3].

$4^{e}$ SÉRIE - TOME $40-2007-\mathrm{N}^{\circ} 1$ 
Lemma 7. - Let $a, q \in \mathbb{Z}$ be such that $q>0$, and let $t_{1}, t_{2} \in \mathbb{R}$ such that $t_{2} \geqslant t_{1}$. Then

$$
\#\left\{t_{1}<n \leqslant t_{2}: n \equiv a(\bmod q)\right\}=\frac{t_{2}-t_{1}}{q}+r\left(t_{1}, t_{2} ; a, q\right),
$$

where

$$
r\left(t_{1}, t_{2} ; a, q\right)=\psi\left(\frac{t_{1}-a}{q}\right)-\psi\left(\frac{t_{2}-a}{q}\right) .
$$

In relation to this result we shall need some control over the average order of the function $\psi(g(x, y) / q)$, for certain real-valued functions $g$, as we range over integers $x, y$ that are restricted to certain intervals and that satisfy a certain congruence relation modulo $q$. The simplest scenario is when $g(x, y)$ is actually a polynomial in one variable, in which case we shall make use of the following result [3, Lemma 5], established by combining a Fourier series expansion for $\psi$ with standard bounds for the quadratic Gauss sum.

LemmA 8. - Let $\varepsilon>0$ and let $t \in \mathbb{R}$. Then for any $a, q \in \mathbb{Z}$ such that $q>0$ and $\operatorname{gcd}(a, q)=1$, we have

$$
\sum_{\substack{y=1 \\ \operatorname{gcd}(y, q)=1}}^{q} \psi\left(\frac{t-a y^{2}}{q}\right) \ll_{\varepsilon} q^{1 / 2+\varepsilon} .
$$

We shall also need to examine the average order of $\psi(g(x, y) / q)$ for the more complicated case in which $g(x, y)=f(x)-x y$ for a suitable function $f$. More precisely, given $a, b, c, q \in \mathbb{Z}$ such that $q>0$ and $\operatorname{gcd}(a b c, q)=1$, and an interval $I \subset \mathbb{R}$, we shall want to study the sum

$$
S_{I}(f, q)=S_{I}(f, q ; a, b, c):=\sum_{\substack{x \in \mathbb{Z} \cap I \\ \operatorname{gcd}(x, q)=1}} \sum_{\substack{y=1 \\ a y^{2} \equiv b x(\bmod q)}}^{q} \psi\left(\frac{f(x)-c x y}{q}\right),
$$

for suitable real-valued functions $f$ on $I$. Our estimates for $S_{I}(f, q)$ will depend upon the work in the previous section, and we shall eventually obtain two distinct estimates according to whether we are in a position to apply Lemma 5 or Lemma 6 . We begin however by recording the following "trivial" bound for (4.1), which follows from the fact that for fixed integers $a, b, x$ such that $\operatorname{gcd}(a b x, q)=1$, there are $\mathrm{O}_{\varepsilon}\left(q^{\varepsilon}\right)$ possible solutions modulo $q$ of the congruence $a y^{2} \equiv b x(\bmod q)$.

Lemma 9. - Let $I \subset \mathbb{R}$ be an interval and suppose that $a, b, c, q \in \mathbb{Z}$ such that $q>0$ and $\operatorname{gcd}(a b c, q)=1$. Then for any real-valued function $f$ on I we have

$$
S_{I}(f, q) \ll_{\varepsilon} q^{\varepsilon} \mathfrak{m}(I),
$$

where $\mathfrak{m}(I)$ is given by (3.11).

The starting point for a more sophisticated treatment of $S_{I}(f, q)$ is the trigonometric formula [19] for $\psi$, that is due to Vaaler. For any $t \in \mathbb{R}$, and any $H \geqslant 1$, this implies that

$$
\sum_{0<|h| \leqslant H} c_{h}^{-} e(h t)+\mathrm{O}\left(\frac{1}{H}\right) \leqslant \psi(t) \leqslant \sum_{0<|h| \leqslant H} c_{h}^{+} e(h t)+\mathrm{O}\left(\frac{1}{H}\right),
$$


for certain coefficients $c_{h}^{-}, c_{h}^{+} \ll 1 /|h|$. Arguing as above we therefore deduce that

$$
S_{I}(f, q) \ll_{\varepsilon} \frac{q^{\varepsilon} \mathfrak{m}(I)}{H}+\sum_{h=1}^{H} \frac{1}{h}\left|T_{I}(f, q ; h)\right|,
$$

in the notation of (3.11), where

$$
T_{I}(f, q ; h)=\sum_{\substack{x \in I \cap \mathbb{Z} \\ \operatorname{gcd}(x, q)=1}} \sum_{\substack{y=1 \\ a y^{2} \equiv b x(\bmod q)}}^{q} e_{q}(h f(x)-\operatorname{ch} x y) .
$$

Extending the summation over $x$ to a complete set of residues modulo $q$, we obtain

$$
\begin{aligned}
T_{I}(f, q ; h)= & \sum_{\substack{u=1 \\
\operatorname{gcd}(u, q)=1}}^{q} \sum_{\substack{x \in I \cap \mathbb{Z} \\
q}} \frac{1}{q} \sum_{k=1}^{q} e_{q}(k(u-x)) \\
& \times \sum_{\substack{v=1 \\
a v^{2} \equiv b u(\bmod q)}}^{q} e_{q}(h f(x)-\text { chuv }) \\
= & \frac{1}{q} \sum_{k=1}^{q} A_{I}(q ;-k, h, f) B(q ; h, k),
\end{aligned}
$$

where

$$
B(q ; h, k)=\sum_{\substack{u=1 \\ \operatorname{gcd}(u, q)=1}}^{q} \sum_{\substack{v=1 \\ a v^{2} \equiv b u(\bmod q)}}^{q} e_{q}(k u-c h u v)
$$

and $A_{I}(q ;-k, h, f)$ is given by (3.4). By periodicity, we may replace the summation over $1 \leqslant k \leqslant q$ by a summation over $-q / 2<k \leqslant q / 2$.

On letting $\bar{b}$ denote the multiplicative inverse of $b$ modulo $q$, it is easy to see that

$$
B(q ; h, k)=\sum_{\substack{v=1 \\ \operatorname{gcd}(v, q)=1}}^{q} e_{q}\left(a \bar{b}\left(-c h v^{3}+k v^{2}\right)\right) .
$$

In order to estimate this sum we must first take care to remove any possible common factors between $q$ and the coefficients of $v^{3}$ and $v^{2}$. Since $\operatorname{gcd}(a b c, q)=1$ by assumption, we see that $\operatorname{gcd}(q, a \bar{b} c h, a \bar{b} k)=\operatorname{gcd}(q, h, k)$, whence

$$
T_{I}(f, q ; h)=\sum_{d \mid h, q} \frac{1}{d q^{\prime}} \sum_{\substack{-q^{\prime} / 2<k^{\prime} \leqslant q^{\prime} / 2 \\ \operatorname{gcd}\left(k^{\prime}, h^{\prime}, q^{\prime}\right)=1}} A_{I}\left(q^{\prime} ;-k^{\prime}, h^{\prime}, f\right) B\left(d q^{\prime} ; d h^{\prime}, d k^{\prime}\right) .
$$

Here, we have written $h=d h^{\prime}, k=d k^{\prime}$ and $q=d q^{\prime}$.

$4^{\text {e }}$ SÉRIE - TOME $40-2007-\mathrm{N}^{\circ} 1$ 
We must now consider the sum $B\left(d q^{\prime} ; d h^{\prime}, d k^{\prime}\right)$ in more detail. Each $v$, modulo $q$, can be written uniquely in the form $v=y+q^{\prime} z$ with $1 \leqslant y \leqslant q^{\prime}$ and $1 \leqslant z \leqslant d$. Thus it follows that

$$
\begin{aligned}
B\left(d q^{\prime} ; d h^{\prime}, d k^{\prime}\right) & =\sum_{y=1}^{q^{\prime}} \sum_{\substack{z=1 \\
\operatorname{gcd}\left(y+q^{\prime} z, d q^{\prime}\right)=1}}^{d} e_{q^{\prime}}\left(a \bar{b}\left(-c h^{\prime} y^{3}+k^{\prime} y^{2}\right)\right) \\
& =\sum_{\substack{y=1 \\
\operatorname{gcd}\left(y, q^{\prime}\right)=1}}^{q^{\prime}} e_{q^{\prime}}\left(a \bar{b}\left(-c h^{\prime} y^{3}+k^{\prime} y^{2}\right)\right) N\left(d ; q^{\prime}, y\right),
\end{aligned}
$$

where $N\left(d ; q^{\prime}, y\right)$ is the number of positive integers $z \leqslant d$ for which $y+q^{\prime} z$ is coprime to $d$. But then it is clear that

$$
\begin{aligned}
N\left(d ; q^{\prime}, y\right)= & \sum_{\ell \mid d} \mu(\ell) \#\left\{1 \leqslant z \leqslant d: q^{\prime} z \equiv-y(\bmod \ell)\right\} \\
= & \sum_{\substack{\ell \mid d \\
\operatorname{gcd}\left(\ell, q^{\prime}\right)=1}} \mu(\ell) \sum_{t=1}^{d / \ell} \#\left\{1 \leqslant s \leqslant \ell: q^{\prime} s \equiv-y(\bmod \ell)\right\} \\
= & d \sum_{\substack{\ell \mid d \\
\operatorname{gcd}\left(\ell, q^{\prime}\right)=1}} \frac{\mu(\ell)}{\ell}=f\left(d, q^{\prime}\right),
\end{aligned}
$$

say. In particular we have

$$
f\left(d, q^{\prime}\right)=d \phi^{*}(d) / \phi^{*}\left(\operatorname{gcd}\left(d, q^{\prime}\right)\right) \leqslant d
$$

where

$$
\phi^{*}(n):=\prod_{p \mid n}(1-1 / p) .
$$

Thus $B\left(d q^{\prime} ; d h^{\prime}, d k^{\prime}\right)=f\left(d, q^{\prime}\right) B\left(q^{\prime}, h^{\prime}, k^{\prime}\right)$, and so

$$
T_{I}(f, q ; h) \ll \sum_{d \mid h, q} \frac{1}{q^{\prime}} \sum_{\begin{array}{c}
-q^{\prime} / 2<k^{\prime} \leqslant q^{\prime} / 2 \\
\operatorname{gcd}\left(k^{\prime}, h^{\prime}, q^{\prime}\right)=1
\end{array}}\left|A_{I}\left(q^{\prime} ;-k^{\prime}, h^{\prime}, f\right)\right|\left|B\left(q^{\prime} ; h^{\prime}, k^{\prime}\right)\right| .
$$

We now break the inner sum over $k^{\prime}$ into two sums: the single term arising from $k^{\prime}=0$ and the summation over $-q^{\prime} / 2<k^{\prime} \leqslant q^{\prime} / 2$ such that $k^{\prime} \neq 0$.

We begin by handling the overall contribution from the term $k^{\prime}=0$. But then it follows from (3.5) that

$$
A_{I}\left(q^{\prime} ; 0, h^{\prime}, f\right) \ll \mathfrak{m}(I)
$$

and from Lemma 2 that

$$
B\left(q^{\prime} ; h^{\prime}, 0\right) \ll_{\varepsilon} q^{\prime 2 / 3+\varepsilon} .
$$

Here we have used the fact that $\operatorname{gcd}\left(k^{\prime}, h^{\prime}, q^{\prime}\right)=\operatorname{gcd}\left(h^{\prime}, q^{\prime}\right)=1$. Combining these two estimates we therefore obtain the overall contribution

$$
\ll_{\varepsilon} q^{\varepsilon} \sum_{d \mid h, q} \frac{\mathfrak{m}(I)}{(q / d)^{1 / 3}} \ll_{\varepsilon} \frac{q^{2 \varepsilon} \operatorname{gcd}(h, q)^{1 / 3} \mathfrak{m}(I)}{q^{1 / 3}},
$$


to the right-hand side of (4.4).

In order to handle the remaining contribution, our argument bifurcates according to which of Lemmas 5 or 6 we apply to estimate $A_{I}\left(q^{\prime} ;-k^{\prime}, h^{\prime}, f\right)$. In either case we may clearly deduce from Lemma 4 that

$$
B\left(q^{\prime} ; h^{\prime}, k^{\prime}\right) \ll_{\varepsilon} q^{1 / 2+\varepsilon} \operatorname{gcd}\left(k^{\prime}, q^{\prime}\right) .
$$

We begin with an application of Lemma 5, for which we shall assume that $f \in C^{1}\left(I ; \lambda_{0}\right)$ for a certain value of $\lambda_{0} \geqslant 1$. Thus it follows that

$$
A_{I}\left(q^{\prime} ;-k^{\prime}, h^{\prime}, f\right) \ll \frac{q^{\prime}}{k^{\prime}}\left(1+\frac{h \lambda_{0}}{q}\right),
$$

since $0<\left|k^{\prime}\right| \leqslant q^{\prime} / 2$, whence

$$
\sum_{\substack{-q^{\prime} / 2<k^{\prime} \leqslant q^{\prime} / 2 \\ \operatorname{gcd}\left(k^{\prime}, h^{\prime}, q^{\prime}\right)=1 \\ k^{\prime} \neq 0}}\left|A_{I}\left(q^{\prime} ;-k^{\prime}, h^{\prime}, f\right)\right|\left|B\left(q^{\prime} ; h^{\prime}, k^{\prime}\right)\right| \ll_{\varepsilon} q^{\prime 3 / 2+2 \varepsilon}\left(1+\frac{h \lambda_{0}}{q}\right) .
$$

Here we have used the trivial observation that

$$
\sum_{1 \leqslant a \leqslant A} \frac{\operatorname{gcd}(a, b)}{a} \leqslant \sum_{d \mid b} d \sum_{1 \leqslant a \leqslant A / d} \frac{1}{a d} \ll \tau(b) \log A,
$$

for any $A \geqslant 2$ and any $b \in \mathbb{N}$, together with the upper bound $\tau(n)=\mathrm{O}_{\varepsilon}\left(n^{\varepsilon}\right)$ for the divisor function. We therefore obtain the overall contribution

$$
\ll_{\varepsilon} \sum_{d \mid h, q} q^{1 / 2+2 \varepsilon}\left(1+\frac{h \lambda_{0}}{q}\right) \ll_{\varepsilon} q^{1 / 2+3 \varepsilon}\left(1+\frac{h \lambda_{0}}{q}\right),
$$

to the right-hand side of (4.4) from this case. Alternatively, let $j, \lambda_{0}, \lambda_{1}, \lambda_{2} \in \mathbb{R}$ such that (3.10) holds, and suppose that $f \in C^{2}\left(I ; \lambda_{0}, \lambda_{1}, \lambda_{2}, j\right)$. Then it follows that

$$
A_{I}\left(q^{\prime} ;-k^{\prime}, h^{\prime}, f\right) \ll \frac{q^{\prime}}{k^{\prime}}\left(1+\lambda_{1} E\right),
$$

where

$$
E=\frac{h^{1 / 2}}{\lambda_{2}^{1 / 2} q^{1 / 2}}+\frac{h^{3 / 2} j \lambda_{2}^{1 / 2} \mathfrak{m}(I)}{q^{3 / 2}}+\frac{h^{2} \lambda_{0}}{q^{2}} .
$$

We may combine this with (4.6) and (4.7) to obtain the overall contribution

$$
\sum_{d \mid h, q} \frac{1}{q^{\prime}} \sum_{\substack{-q^{\prime} / 2<k^{\prime} \leqslant q^{\prime} / 2 \\ \operatorname{gcd}\left(k^{\prime}, h^{\prime}, q^{\prime}\right)=1 \\ k^{\prime} \neq 0}}\left|A_{I}\left(q^{\prime} ;-k^{\prime}, h^{\prime}, f\right)\right|\left|B\left(q^{\prime} ; h^{\prime}, k^{\prime}\right)\right| \ll_{\varepsilon} q^{1 / 2+3 \varepsilon}\left(1+\lambda_{1} E\right)
$$

to the right-hand side of (4.4) from this case.

$4^{\mathrm{e}}$ SÉRIE - TOME $40-2007-\mathrm{N}^{\circ} 1$ 
Let us begin by drawing together (4.5) and (4.8) in (4.4), before then inserting the resulting bound into (4.2). In view of (4.7) we have shown that

$$
\begin{aligned}
S_{I}(f, q) & \ll_{\varepsilon} \frac{q^{\varepsilon} \mathfrak{m}(I)}{H}+q^{3 \varepsilon} \sum_{h=1}^{H}\left(\frac{\operatorname{gcd}(h, q) \mathfrak{m}(I)}{h q^{1 / 3}}+\frac{q^{1 / 2}}{h}+\frac{\lambda_{0}}{q^{1 / 2}}\right) \\
& \ll_{\varepsilon} q^{3 \varepsilon} H^{\varepsilon}\left(\frac{\mathfrak{m}(I)}{H}+\frac{\mathfrak{m}(I)}{q^{1 / 3}}+q^{1 / 2}+H \frac{\lambda_{0}}{q^{1 / 2}}\right),
\end{aligned}
$$

for any $f \in C^{1}\left(I ; \lambda_{0}\right)$ and any $H \geqslant 1$. Suppose first that $\mathfrak{m}(I) q^{1 / 2} \geqslant \lambda_{0}$. Then we may select

$$
H=\frac{\mathfrak{m}(I)^{1 / 2} q^{1 / 4}}{\lambda_{0}^{1 / 2}}
$$

to get

$$
S_{I}(f, q) \ll_{\varepsilon} q^{4 \varepsilon} \mathfrak{m}(I)^{\varepsilon}\left(\frac{\mathfrak{m}(I)}{q^{1 / 3}}+q^{1 / 2}+\frac{\mathfrak{m}(I)^{1 / 2} \lambda_{0}^{1 / 2}}{q^{1 / 4}}\right) .
$$

Alternatively, if $\mathfrak{m}(I) q^{1 / 2} \leqslant \lambda_{0}$ we employ the trivial estimate Lemma 9 for $S_{I}(f, q)$, to conclude that

$$
S_{I}(f, q) \ll_{\varepsilon} q^{\varepsilon} \mathfrak{m}(I) \ll_{\varepsilon} q^{\varepsilon} \frac{\mathfrak{m}(I)^{1 / 2} \lambda_{0}^{1 / 2}}{q^{1 / 4}} .
$$

On combining these two estimates and redefining the choice of $\varepsilon$, we have therefore established the following result.

Lemma 10. - Let $I \subset \mathbb{R}$ be an interval and let $\lambda_{0} \geqslant 1$. Suppose that $a, b, c, q \in \mathbb{Z}$ such that $q>0$ and $\operatorname{gcd}(a b c, q)=1$, and let $f \in C^{1}\left(I ; \lambda_{0}\right)$. Then we have

$$
S_{I}(f, q) \ll_{\varepsilon} q^{\varepsilon} \mathfrak{m}(I)^{\varepsilon}\left(q^{1 / 2}+\frac{\mathfrak{m}(I)}{q^{1 / 3}}+\frac{\lambda_{0}^{1 / 2} \mathfrak{m}(I)^{1 / 2}}{q^{1 / 4}}\right),
$$

where $\mathfrak{m}(I)$ is given by (3.11).

We may obtain an alternative estimate for $S_{I}(f, q)$ by drawing together (4.5) and (4.10) in (4.4), when $f \in C^{2}\left(I ; \lambda_{0}, \lambda_{1}, \lambda_{2}, j\right)$ for $j, \lambda_{0}, \lambda_{1}, \lambda_{2} \in \mathbb{R}$ such that (3.10) holds. On inserting the resulting estimate for (4.4) into (4.2) we conclude that

$$
S_{I}(f, q) \ll_{\varepsilon} \frac{q^{\varepsilon} \mathfrak{m}(I)}{H}+q^{3 \varepsilon} \sum_{h=1}^{H}\left(\frac{\operatorname{gcd}(h, q) \mathfrak{m}(I)}{h q^{1 / 3}}+\frac{q^{1 / 2}}{h}+\frac{q^{1 / 2} \lambda_{1}}{h} E\right),
$$

where $E$ is given by (4.9). But then (4.7) yields

$$
S_{I}(f, q) \ll_{\varepsilon} q^{3 \varepsilon} H^{\varepsilon}\left(\frac{\mathfrak{m}(I)}{H}+\frac{\mathfrak{m}(I)}{q^{1 / 3}}+q^{1 / 2}+F\right),
$$

where

$$
F=\frac{H^{1 / 2} \lambda_{1}}{\lambda_{2}^{1 / 2}}+\frac{H^{3 / 2} j \lambda_{1} \lambda_{2}^{1 / 2} \mathfrak{m}(I)}{q}+\frac{H^{2} \lambda_{0} \lambda_{1}}{q^{3 / 2}} .
$$

ANNALES SCIENTIFIQUES DE L'ÉCOLE NORMALE SUPÉRIEURE 
Suppose first that $\lambda_{2} \mathfrak{m}(I)^{2} \geqslant \lambda_{1}^{2}$. Then we may select

$$
H=\frac{\lambda_{2}^{1 / 3} \mathfrak{m}(I)^{2 / 3}}{\lambda_{1}^{2 / 3}}
$$

and it follows that

$$
S_{I}(f, q) \ll_{\varepsilon} q^{4 \varepsilon}\left(q^{1 / 2}+\frac{\mathfrak{m}(I)}{q^{1 / 3}}+\frac{\lambda_{1}^{2 / 3} \mathfrak{m}(I)^{1 / 3}}{\lambda_{2}^{1 / 3}}+\frac{j \lambda_{2} \mathfrak{m}(I)^{2}}{q}+\frac{\lambda_{0} \lambda_{2}^{2 / 3} \mathfrak{m}(I)^{4 / 3}}{\lambda_{1}^{1 / 3} q^{3 / 2}}\right) .
$$

Alternatively, if $\lambda_{2} \mathfrak{m}(I)^{2} \leqslant \lambda_{1}^{2}$ then Lemma 9 implies that

$$
S_{I}(f, q) \ll_{\varepsilon} q^{\varepsilon} \mathfrak{m}(I) \ll_{\varepsilon} q^{\varepsilon} \frac{\lambda_{1}^{2 / 3} \mathfrak{m}(I)^{1 / 3}}{\lambda_{2}^{1 / 3}} .
$$

On combining these two estimates and redefining the choice of $\varepsilon$, we have therefore established the following result.

LEMma 11. - Let $I \subset \mathbb{R}$ be an interval and let $j, \lambda_{0}, \lambda_{1}, \lambda_{2} \in \mathbb{R}$ such that (3.10) holds. Suppose that $a, b, c, q \in \mathbb{Z}$ such that $q>0$ and $\operatorname{gcd}(a b c, q)=1$, and let $f \in C^{2}\left(I ; \lambda_{0}, \lambda_{1}, \lambda_{2}, j\right)$. Then we have

$$
S_{I}(f, q) \ll_{\varepsilon} q^{\varepsilon}\left(q^{1 / 2}+\frac{\mathfrak{m}(I)}{q^{1 / 3}}+\frac{\lambda_{1}^{2 / 3} \mathfrak{m}(I)^{1 / 3}}{\lambda_{2}^{1 / 3}}+\frac{j \lambda_{2} \mathfrak{m}(I)^{2}}{q}+\frac{\lambda_{0} \lambda_{2}^{2 / 3} \mathfrak{m}(I)^{4 / 3}}{\lambda_{1}^{1 / 3} q^{3 / 2}}\right),
$$

where $\mathfrak{m}(I)$ is given by (3.11).

\section{The real-valued functions $g_{1}$ and $g_{2}$}

The purpose of this section is to introduce and analyse a number of real-valued functions that play a pivotal role in subsequent sections. In fact they will arise in $\S 7$ as boundary curves for the heights of the variables to be introduced during our passage to the universal torsor. It is precisely to some of these functions that we will ultimately apply the results of the previous section.

We begin by introducing a function $g_{1}:[0,1] \rightarrow \mathbb{R}$ on the unit interval, given by

$$
g_{1}(v):=-\left(\min \left\{1 / v^{4}, 1+1 / v^{2}\right\}\right)^{1 / 3} .
$$

Next we introduce functions $g_{21}, g_{22}:(-\infty, 1] \times[0,1] \rightarrow \mathbb{R}$, which are given by

$$
g_{21}(u, v):= \begin{cases}0, & \text { if }-1 \leqslant u \leqslant 1 \\ \sqrt{-1-u^{3}}, & \text { if } u \leqslant-1\end{cases}
$$

and

$$
g_{22}(u, v):= \begin{cases}\sqrt{1-u^{3}}, & \text { if }-\left(1 / v^{2}-1\right)^{1 / 3} \leqslant u \leqslant 1 \\ 1 / v, & \text { if } u \leqslant-\left(1 / v^{2}-1\right)^{1 / 3}\end{cases}
$$

respectively. Finally let us define the function $g_{2}: \mathbb{R}^{2} \rightarrow \mathbb{R}$, by

$$
g_{2}(u, v):= \begin{cases}g_{22}(u, v)-g_{21}(u, v), & \text { if } g_{1}(v) \leqslant u \leqslant 1 \text { and } v \in[0,1] \\ 0, & \text { otherwise }\end{cases}
$$

$4^{e}$ SÉRIE - TOME $40-2007-\mathrm{N}^{\circ} 1$ 
Then we clearly have

$$
\begin{aligned}
g_{2}(u, v) & =\sqrt{\min \left\{1 / v^{2}, 1-u^{3}\right\}}-\sqrt{\max \left\{0,-1-u^{3}\right\}} \\
& =\int_{\left\{t \in \mathbb{R}: 0 \leqslant t v \leqslant 1,\left|t^{2}+u^{3}\right| \leqslant 1\right\}} \mathrm{d} t,
\end{aligned}
$$

for $g_{1}(v) \leqslant u \leqslant 1$ and $v \in[0,1]$.

Write $D_{i} g_{2}(u, v)$ for the partial derivative of $g_{2}$ with respect to the $i$-th variable, for $i=1,2$. Then we shall need to know something about the behaviour of $D_{1} g_{2}(u, v)$ and $\left|D_{1} g_{2}(u, v)\right|$ as we integrate over all values of $u$. For this it will be necessary to calculate $g_{2}(u, v)$ explicitly. It suffices to restrict attention to $u, v$ such that $g_{1}(v) \leqslant u \leqslant 1$ and $v \in[0,1]$, since $g_{2}(u, v)$ is defined to be zero in all other cases. Thus one easily combines (5.2), (5.3) and (5.5) to deduce that

$$
g_{2}(u, v)=\sqrt{1-u^{3}},
$$

if $0 \leqslant u \leqslant 1$. If $2^{-1 / 2}<v \leqslant 1$ and $1 / v^{2}-1<-u^{3} \leqslant 1$, then

$$
g_{2}(u, v)=1 / v
$$

If $0 \leqslant-u^{3} \leqslant \min \left\{1 / v^{2}-1,1\right\}$, we have

$$
g_{2}(u, v)=\sqrt{1-u^{3}}
$$

while if $0<v \leqslant 2^{-1 / 2}$ and $1 \leqslant-u^{3} \leqslant 1 / v^{2}-1$, we have

$$
g_{2}(u, v)=\sqrt{1-u^{3}}-\sqrt{-1-u^{3}}=\frac{2}{\sqrt{1-u^{3}}+\sqrt{-1-u^{3}}} .
$$

Finally,

$$
g_{2}(u, v)=1 / v-\sqrt{-1-u^{3}}
$$

if $\max \left\{1 / v^{2}-1,1\right\}<-u^{3} \leqslant \min \left\{1 / v^{2}+1,1 / v^{4}\right\}$. It follows from these calculations that

$$
\int_{-\infty}^{\infty} D_{1} g_{2}(u, v) \mathrm{d} u=g_{2}(1, v)-g_{2}\left(g_{1}(v), v\right)=-g_{2}\left(g_{1}(v), v\right),
$$

since $g_{2}(1, v)=0$ for any $v \in \mathbb{R}$. Furthermore it is now straightforward to conclude that

$$
\int_{-\infty}^{\infty}\left|D_{1} g_{2}(u, v)\right| \mathrm{d} u \ll 1
$$

for any $v \in \mathbb{R}$. The final fact that we shall need to highlight here is the elementary equality

$$
g_{2}\left(g_{1}(v), v\right)=0 \quad\left(0<v \leqslant 2^{-1 / 2}\right) .
$$

\section{Proof of Theorem 1: preliminaries}

We are now ready to commence the proof of Theorem 1 in earnest, for which it is necessary to introduce some more notation. For any $n \geqslant 2$ we let $Z^{n+1}$ denote the set of primitive vectors 
in $\mathbb{Z}^{n+1}$, where $\mathbf{v}=\left(v_{0}, \ldots, v_{n}\right) \in \mathbb{Z}^{n+1}$ is said to be primitive if $\operatorname{gcd}\left(v_{0}, \ldots, v_{n}\right)=1$. Finally we shall let $\mathbb{Z}_{*}^{n+1}$ (resp. $Z_{*}^{n+1}$ ) denote the set of vectors $\mathbf{v} \in \mathbb{Z}^{n+1}$ (resp. $\mathbf{v} \in Z^{n+1}$ ) such that $v_{0} \cdots v_{n} \neq 0$.

In this section we shall establish a preliminary estimate for $N_{U, H}(B)$, which paves the way towards the universal torsor calculation in the following section. If $x=[\mathbf{x}] \in \mathbb{P}^{3}(\mathbb{Q})$ is represented by the vector $\mathbf{x} \in Z^{4}$, then $H(x)=|\mathbf{x}|=\max _{0 \leqslant i \leqslant 3}\left|x_{i}\right|$. Thus it is easy to see that

$$
N_{U, H}(B)=\frac{1}{2} \#\left\{\mathbf{x} \in Z^{4}:|\mathbf{x}| \leqslant B, x_{1} x_{2}^{2}+x_{2} x_{0}^{2}+x_{3}^{3}=0\right\},
$$

since $\mathbf{x}$ and $-\mathbf{x}$ represent the same point in $\mathbb{P}^{3}$. We proceed by considering the contribution to $N_{U, H}(B)$ from vectors $\mathbf{x} \in Z^{4}$ which contain zero components. Define the set

$$
\mathcal{E}(B):=\left\{\mathbf{x} \in Z_{*}^{4}: x_{0}, x_{2}>0,|\mathbf{x}| \leqslant B, f(\mathbf{x})=0\right\},
$$

where $f(\mathbf{x})=x_{1} x_{2}^{2}+x_{2} x_{0}^{2}+x_{3}^{3}$. Then we have the following result.

LEMMA 12. - Let $B \geqslant 1$. Then we have

$$
N_{U, H}(B)=2 \# \mathcal{E}(B)+\frac{12}{\pi^{2}} B+\mathrm{O}\left(B^{2 / 3}\right) .
$$

Proof. - Suppose that $\mathrm{x} \in Z^{4}$ is a vector such that

$$
x_{0} x_{1}=0, \quad\left|x_{0}\right|,\left|x_{1}\right|,\left|x_{2}\right|,\left|x_{3}\right| \leqslant B,
$$

and $x=\left[x_{0}, \ldots, x_{3}\right] \in S$, where $S$ denotes the surface $f(\mathbf{x})=0$. If $x_{0}=0$ then $x_{1} x_{2}^{2}+x_{3}^{3}=0$, and so we are interested in vectors of the shape $\left(x_{1}, x_{2}, x_{3}\right)= \pm\left(a^{3}, b^{3},-a b^{2}\right)$ for coprime $a, b \in \mathbb{N}$. Such points therefore contribute $\mathrm{O}\left(B^{2 / 3}\right)$ to $N_{U, H}(B)$. The case in which $x_{1}=0$ is similar. Suppose now that $x \in S$ is represented by a vector $\mathbf{x} \in Z^{4}$ such that

$$
x_{2}=0, \quad\left|x_{0}\right|,\left|x_{1}\right|,\left|x_{3}\right| \leqslant B .
$$

Then necessarily $x_{3}=0$ and the point is not to be counted, since it lies on the unique line in $S$. Finally we suppose that $\mathbf{x} \in Z^{4}$ satisfies

$$
x_{3}=0, \quad 0<\left|x_{0}\right|,\left|x_{1}\right|,\left|x_{2}\right| \leqslant B,
$$

and $\left[x_{0}, x_{1}, x_{2}, 0\right] \in U$. Then we must have $x_{0}^{2}+x_{1} x_{2}=0$, so that $\mathbf{x}=\left( \pm a b,-a^{2}, b^{2}, 0\right)$ or $\mathbf{x}=$ $\left( \pm a b, a^{2},-b^{2}, 0\right)$ for coprime $a, b \in \mathbb{N}$. Now the number of coprime $a, b \in \mathbb{N}$ such that $a, b \leqslant Y$ is $6 Y^{2} / \pi^{2}+\mathrm{O}(Y \log Y)$. Hence the contribution from this case is $12 B / \pi^{2}+\mathrm{O}\left(B^{1 / 2} \log B\right)$, whence

$$
N_{U, H}(B)=\frac{1}{2} \#\left\{\mathbf{x} \in Z_{*}^{4}:|\mathbf{x}| \leqslant B, f(\mathbf{x})=0\right\}+\frac{12}{\pi^{2}} B+\mathrm{O}\left(B^{2 / 3}\right) .
$$

We complete the proof of Lemma 12 by choosing $x_{0}>0$ and $x_{2}>0$, as we clearly may.

\section{Proof of Theorem 1: the universal torsor}

The purpose of this section is to establish a bijection between the rational points on the open subset $U$ of the cubic surface $S$, and the integral points on the universal torsor above $\widetilde{S}$, which are

$4^{\mathrm{e}}$ SÉRIE - TOME $40-2007-\mathrm{N}^{\circ} 1$ 
subject to a number of coprimality conditions. Along the way we shall introduce new variables

$$
\xi_{1}, \xi_{2}, \xi_{3}, \xi_{\ell}, \xi_{4}, \xi_{5}, \xi_{6}, \tau_{1}, \tau_{2}, \tau_{\ell}
$$

and it will be convenient to henceforth write $\boldsymbol{\xi}=\left(\xi_{1}, \xi_{2}, \xi_{3}, \xi_{\ell}, \xi_{4}, \xi_{5}, \xi_{6}\right)$ and $\boldsymbol{\tau}=\left(\tau_{1}, \tau_{2}, \tau_{\ell}\right)$. Furthermore we shall make frequent use of the notation

$$
\xi^{\left(n_{1}, n_{2}, n_{3}, n_{\ell}, n_{4}, n_{5}, n_{6}\right)}:=\xi_{1}^{n_{1}} \xi_{2}^{n_{2}} \xi_{3}^{n_{3}} \xi_{\ell}^{n_{\ell}} \xi_{4}^{n_{4}} \xi_{5}^{n_{5}} \xi_{6}^{n_{6}},
$$

in all that follows.

Hassett and Tschinkel $[10, \S 3]$ have calculated the Cox ring of $\widetilde{S}$ as being given by

$$
\operatorname{Cox}(\widetilde{S})=\operatorname{Spec}(\mathbb{Q}[\boldsymbol{\xi}, \boldsymbol{\tau}] /(T(\boldsymbol{\xi}, \boldsymbol{\tau}))),
$$

where

$$
T(\boldsymbol{\xi}, \boldsymbol{\tau}):=\tau_{\ell} \xi_{\ell}^{3} \xi_{4}^{2} \xi_{5}+\tau_{2}^{2} \xi_{2}+\tau_{1}^{3} \xi_{1}^{2} \xi_{3} .
$$

The universal torsor $\mathcal{T}$ is an open subset of the affine hypersurface

$$
\tau_{\ell} \xi_{\ell}^{3} \xi_{4}^{2} \xi_{5}+\tau_{2}^{2} \xi_{2}+\tau_{1}^{3} \xi_{1}^{2} \xi_{3}=0
$$

together with a map $\Psi: \mathcal{T} \rightarrow S$, given by

$$
\begin{aligned}
& \Psi^{*}\left(x_{0}\right)=\xi^{(1,2,2,0,1,2,3)} \tau_{2}, \\
& \Psi^{*}\left(x_{1}\right)=\tau_{\ell}, \\
& \Psi^{*}\left(x_{2}\right)=\xi^{(2,3,4,3,4,5,6)}, \\
& \Psi^{*}\left(x_{3}\right)=\xi^{(2,2,3,1,2,3,4)} \tau_{1} .
\end{aligned}
$$

Below we shall use our own methods to show how $\Psi$ gives a bijection between $U(\mathbb{Q})$ and the integral points on the hypersurface (7.1), subject to certain coprimality conditions. Recall the notation introduced in $\S 2$ to describe the Picard group $\operatorname{Pic}(\widetilde{S})$ of $\widetilde{S}$. Then it is interesting to remark that the variables $\xi_{1}, \ldots, \xi_{6}, \xi_{\ell}$ arise as non-zero sections that generate $\Gamma\left(E_{1}\right), \ldots, \Gamma\left(E_{6}\right), \Gamma(\ell)$, respectively, where $\Gamma(A)=H^{0}(\widetilde{S}, A)$ for any divisor class $A \in \operatorname{Pic}(\widetilde{S})$. The variables $\tau_{1}, \tau_{2}, \tau_{\ell}$ are certain extra sections that are needed to generate the full Cox ring. These are chosen to be sections of the nef divisor class that is dual to $F_{1}, F_{2}, \ell$, respectively. The interested reader should consult the calculation of Hassett and Tschinkel for further details.

We now turn to the task of establishing the bijection. Let us define the set

$$
\mathcal{E}:=\left\{\mathbf{x} \in Z_{*}^{4}: x_{0}, x_{2}>0, x_{1} x_{2}^{2}+x_{2} x_{0}^{2}+x_{3}^{3}=0\right\},
$$

so that $\mathcal{E}=\mathcal{E}(\infty)$ in the notation of (6.1). We begin by demonstrating a bijection between this set and the set $\mathcal{T}_{1}$ of $(\boldsymbol{\xi}, \boldsymbol{\tau}) \in \mathbb{N}^{7} \times \mathbb{Z}_{*} \times \mathbb{N} \times \mathbb{Z}_{*}$ satisfying $T(\boldsymbol{\xi}, \boldsymbol{\tau})=0$, such that

$$
\left|\mu\left(\xi_{1} \xi_{2} \xi_{3} \xi_{4} \xi_{5}\right)\right|=1, \quad \operatorname{gcd}\left(\tau_{1}, \xi_{2} \xi_{\ell} \xi_{4} \xi_{5}\right)=1,
$$

and

$$
\operatorname{gcd}\left(\tau_{2}, \xi_{1} \xi_{3}\right)=\operatorname{gcd}\left(\tau_{\ell}, \xi_{4} \xi_{5} \xi_{6}\right)=1 .
$$

This is achieved in the following result. 
Lemma 13. - The map $\Psi$ induces a bijection between $\mathcal{T}_{1}$ and $\mathcal{E}$.

Proof. - The idea of the proof is simply to go through a series of elementary considerations about the divisibility properties enjoyed by elements of the set $\mathcal{E}$. At each stage we shall replace the original variables by products of new ones which fulfil certain auxiliary conditions, and which will be uniquely determined by the process.

To begin with, let $\mathbf{x} \in \mathcal{E}$ and note that $x_{2} \mid x_{3}^{3}$. We may therefore write $x_{2}=y_{1} y_{2}^{2} y_{3}^{3}$ with $y_{1}, y_{2}, y_{3} \in \mathbb{N}$ such that $\left|\mu\left(y_{1} y_{2}\right)\right|=1$, where each triple occurrence of a prime factor of $x_{2}$ is put in $y_{3}$ and each double occurrence in $y_{2}$. Then $x_{3}=y_{1} y_{2} y_{3} z$ must hold for a suitable $z \in \mathbb{Z}_{*}$. Substituting into $f$ and dividing by $y_{1} y_{2}^{2} y_{3}^{3}$ give the new equation

$$
f_{1}\left(x_{0}, x_{1}, y_{1}, y_{2}, y_{3}, z\right)=x_{1} y_{1} y_{2}^{2} y_{3}^{3}+x_{0}^{2}+y_{1}^{2} y_{2} z^{3}=0 .
$$

Now $y_{1} y_{2} \mid x_{0}^{2}$, and since $\left|\mu\left(y_{1} y_{2}\right)\right|=1$, we have $y_{1} y_{2} \mid x_{0}$. Write $x_{0}=y_{1} y_{2} w$ for a suitable $w \in \mathbb{N}$. Substituting, and dividing by $y_{1} y_{2}$, we therefore obtain

$$
f_{2}\left(x_{1}, y_{1}, y_{2}, y_{3}, z, w\right)=x_{1} y_{2} y_{3}^{3}+w^{2} y_{1} y_{2}+y_{1} z^{3}=0 .
$$

Since $y_{2} \mid y_{1} z^{3}$ and $\left|\mu\left(y_{1} y_{2}\right)\right|=1$, we must have $y_{2} \mid z$. Writing $z=y_{2} z^{\prime}$, where $z^{\prime} \in \mathbb{Z}_{*}$, yields

$$
f_{3}\left(x_{1}, y_{1}, y_{2}, y_{3}, w, z^{\prime}\right)=x_{1} y_{3}^{3}+w^{2} y_{1}+y_{1} y_{2}^{2}{z^{\prime}}^{3}=0
$$

after dividing by $y_{2}$. Since $y_{1}$ divides our original variables $x_{0}, x_{2}, x_{3}$, it must be coprime to $x_{1}$. Once combined with the fact that $y_{1}$ is square-free, the fact $y_{1} \mid x_{1} y_{3}^{3}$ implies that $y_{1} \mid y_{3}$. Hence $y_{3}=y_{1} y_{3}^{\prime}$, where $y_{3}^{\prime} \in \mathbb{N}$, and we obtain

$$
f_{4}\left(x_{1}, y_{1}, y_{2}, w, z^{\prime}, y_{3}^{\prime}\right)=x_{1} y_{1}^{2}{y_{3}^{\prime}}^{3}+w^{2}+y_{2}^{2}{z^{\prime}}^{3}=0 .
$$

Let $a=\operatorname{gcd}\left(y_{3}^{\prime}, z^{\prime}\right) \in \mathbb{N}$ and write $y_{3}^{\prime}=a y_{3}^{\prime \prime}$ and $z^{\prime}=a z^{\prime \prime}$, for $y_{3}^{\prime \prime} \in \mathbb{N}$ and $z^{\prime \prime} \in \mathbb{Z}_{*}$. This gives

$$
f_{5}\left(x_{1}, y_{1}, y_{2}, w, z^{\prime \prime}, y_{3}^{\prime \prime}, a\right)=x_{1} y_{1}^{2} y_{3}^{\prime \prime 3} a^{3}+w^{2}+y_{2}^{2} z^{\prime \prime 3} a^{3}=0 .
$$

Now $a^{3} \mid w^{2}$. Writing $a=\xi_{6}^{2} \xi_{2}$, for $\xi_{2}, \xi_{6} \in \mathbb{N}$ such that $\left|\mu\left(\xi_{2}\right)\right|=1$, gives $w=w^{\prime} \xi_{6}^{3} \xi_{2}^{2}$ for suitable $w^{\prime} \in \mathbb{N}$. This leads to the equation

$$
f_{6}\left(x_{1}, y_{1}, y_{2}, z^{\prime \prime}, y_{3}^{\prime \prime}, w^{\prime}, \xi_{2}, \xi_{6}\right)=x_{1} y_{1}^{2} y_{3}^{\prime \prime 3}+w^{\prime 2} \xi_{2}+y_{2}^{2} z^{\prime \prime 3}=0 .
$$

Let $\xi_{5}=\operatorname{gcd}\left(y_{3}^{\prime \prime}, w^{\prime}\right) \in \mathbb{N}$ and write $y_{3}^{\prime \prime}=\xi_{\ell} \xi_{5}$ and $w^{\prime}=w^{\prime \prime} \xi_{5}$, for suitable $\xi_{\ell}, w^{\prime \prime} \in \mathbb{N}$. Then

$$
f_{7}\left(x_{1}, y_{1}, y_{2}, z^{\prime \prime}, w^{\prime \prime}, \xi_{2}, \xi_{\ell}, \xi_{5}, \xi_{6}\right)=x_{1} y_{1}^{2} \xi_{\ell}^{3} \xi_{5}^{3}+w^{\prime \prime 2} \xi_{2} \xi_{5}^{2}+y_{2}^{2} z^{\prime \prime 3}=0 .
$$

Since $\operatorname{gcd}\left(y_{3}^{\prime \prime}, z^{\prime \prime}\right)=1$, we also have $\operatorname{gcd}\left(\xi_{\ell} \xi_{5}, z^{\prime \prime}\right)=1$. Therefore $\xi_{5}^{2} \mid y_{2}^{2}$, and so $\xi_{5} \mid y_{2}$. We proceed to write $y_{2}=\xi_{1} \xi_{5}$, with $\xi_{1} \in \mathbb{N}$, and so obtain

$$
f_{8}\left(x_{1}, y_{1}, z^{\prime \prime}, w^{\prime \prime}, \xi_{1}, \xi_{2}, \xi_{\ell}, \xi_{5}, \xi_{6}\right)=x_{1} y_{1}^{2} \xi_{\ell}^{3} \xi_{5}+w^{\prime \prime 2} \xi_{2}+\xi_{1}^{2}{z^{\prime \prime}}^{3}=0
$$

Let $\xi_{3}=\operatorname{gcd}\left(w^{\prime \prime}, y_{1}\right) \in \mathbb{N}$. Since $\left|\mu\left(y_{1} y_{2}\right)\right|=1$, we have $\operatorname{gcd}\left(\xi_{1}, \xi_{3}\right)=1$. Therefore, $\xi_{3} \mid z^{\prime \prime 3}$ and even $\xi_{3} \mid z^{\prime \prime}$. Write $w^{\prime \prime}=\tau_{2} \xi_{3}$ for suitable $\tau_{2} \in \mathbb{N}$, and $y_{1}=\xi_{4} \xi_{3}$ for suitable $\xi_{4} \in \mathbb{N}$, and 
finally $z^{\prime \prime}=\tau_{1} \xi_{3}$ for suitable $\tau_{1} \in \mathbb{Z}_{*}$. Then on writing $x_{1}=\tau_{\ell} \in \mathbb{Z}_{*}$, we therefore obtain the torsor equation $T(\boldsymbol{\xi}, \boldsymbol{\tau})=0$, as given by (7.1).

Now it is easy to check that the substitutions we have made lead to a bijection between the set of $\mathbf{x} \in \mathbb{Z}_{*}^{4}$ such that

$$
x_{0}, x_{2}>0, \quad x_{1} x_{2}^{2}+x_{2} x_{0}^{2}+x_{3}^{3}=0,
$$

and the set of $(\boldsymbol{\xi}, \boldsymbol{\tau}) \in \mathbb{N}^{7} \times \mathbb{Z}_{*} \times \mathbb{N} \times \mathbb{Z}_{*}$ satisfying $T(\boldsymbol{\xi}, \boldsymbol{\tau})=0$, and that this bijection is given by (7.2). In order to complete the proof of the lemma it therefore remains to collect together the coprimality relations that are satisfied by the new variables $\xi, \tau$, and consider how they correspond to the primitivity of the vectors in the set $\mathcal{E}$ under the map $\Psi$.

But on tracing through our argument, one easily checks that we have imposed the coprimality conditions

$$
\begin{aligned}
& \left|\mu\left(y_{1} y_{2}\right)\right|=\left|\mu\left(\xi_{1} \xi_{3} \xi_{4} \xi_{5}\right)\right|=1, \quad\left|\mu\left(\xi_{2}\right)\right|=1, \quad \operatorname{gcd}\left(\tau_{2}, \xi_{4}\right)=1, \\
& \operatorname{gcd}\left(y_{3}^{\prime \prime}, z^{\prime \prime}\right)=\operatorname{gcd}\left(\xi_{\ell} \xi_{5}, \tau_{1} \xi_{3}\right)=1, \quad \operatorname{gcd}\left(\xi_{\ell}, w^{\prime \prime}\right)=\operatorname{gcd}\left(\xi_{\ell}, \tau_{2} \xi_{3}\right)=1,
\end{aligned}
$$

on $\boldsymbol{\xi}, \boldsymbol{\tau}$, together with the condition $\operatorname{gcd}\left(\tau_{\ell}, \xi_{1} \xi_{2} \xi_{3} \xi_{4} \xi_{5} \xi_{6}\right)=1$ that arises from the condition $\operatorname{gcd}\left(x_{0}, x_{1}, x_{2}, x_{3}\right)=1$. A little thought reveals that these conditions can be rewritten as

$$
\begin{aligned}
& \left|\mu\left(\xi_{1} \xi_{3} \xi_{4} \xi_{5}\right)\right|=\left|\mu\left(\xi_{2}\right)\right|=\operatorname{gcd}\left(\xi_{3}, \xi_{\ell}\right)=1 \\
& \operatorname{gcd}\left(\tau_{1}, \xi_{\ell} \xi_{5}\right)=\operatorname{gcd}\left(\tau_{2}, \xi_{\ell} \xi_{4}\right)=\operatorname{gcd}\left(\tau_{\ell}, \xi_{1} \xi_{2} \xi_{3} \xi_{4} \xi_{5} \xi_{6}\right)=1
\end{aligned}
$$

Our final task is therefore to show that, once taken together with (7.1), these coprimality relations are equivalent to the conditions (7.4) and (7.5) that appear in the definition of $\mathcal{T}_{1}$. We content ourselves with checking the reverse implication, the other direction being entirely similar. For this it is clearly enough to show that the relations (7.1), (7.4) and (7.5) together imply that

$$
\operatorname{gcd}\left(\xi_{3}, \xi_{\ell}\right)=\operatorname{gcd}\left(\tau_{2}, \xi_{\ell} \xi_{4}\right)=\operatorname{gcd}\left(\tau_{\ell}, \xi_{1} \xi_{2} \xi_{3}\right)=1 .
$$

Suppose first that there is a common prime factor $p$ of $\xi_{3}, \xi_{\ell}$ in (7.1). Then $p \mid \tau_{2}^{2} \xi_{2}$, which is impossible by (7.4) and (7.5). Hence $\operatorname{gcd}\left(\xi_{3}, \xi_{\ell}\right)=1$. If there is a prime $p$ dividing $\tau_{2}$ and $\xi_{\ell} \xi_{4}$ in (7.1), then $p$ divides $\tau_{1}^{3} \xi_{1}^{2} \xi_{3}$, which is also impossible by (7.4) and (7.5). Finally one deduces that $\tau_{\ell}$ must be coprime to $\xi_{1} \xi_{2} \xi_{3}$ in a similar fashion. This completes the proof of Lemma 13.

During the course of our work it will be convenient to replace the coprimality relations (7.4) in the definition of $\mathcal{T}_{1}$ by relations of the shape

$$
\left|\mu\left(\xi_{2} \xi_{3} \xi_{4} \xi_{5}\right)\right|=1, \quad \operatorname{gcd}\left(\xi_{1}, \xi_{2}\right)=1,
$$

and

$$
\operatorname{gcd}\left(\tau_{1}, \xi_{2} \xi_{3} \xi_{\ell} \xi_{4} \xi_{5} \xi_{6}\right)=1
$$

Thus let us define $\mathcal{T}_{2}$ to be the set of $(\boldsymbol{\xi}, \boldsymbol{\tau}) \in \mathbb{N}^{7} \times \mathbb{Z}_{*} \times \mathbb{N} \times \mathbb{Z}_{*}$ satisfying $T(\boldsymbol{\xi}, \boldsymbol{\tau})=0$, such that (7.5), (7.6) and (7.7) hold. On recalling the definition (7.3) of $\mathcal{E}$, we have the following result.

LEMMA 14. - The map $\Psi$ induces a bijection between $\mathcal{T}_{2}$ and $\mathcal{E}$.

Proof. - In view of Lemma 13 it will suffice to establish a suitable bijection between $\mathcal{T}_{1}$ and $\mathcal{T}_{2}$. Let $(\boldsymbol{\xi}, \boldsymbol{\tau}) \in \mathcal{T}_{1}$. We decompose the coordinates into their prime factors by writing

$$
\xi_{i}=\prod_{p} p^{m_{i, p}}, \quad \tau_{1}= \pm \prod_{p} p^{n_{1, p}}, \quad \tau_{2}=\prod_{p} p^{n_{2, p}}, \quad \tau_{\ell}= \pm \prod_{p} p^{n_{\ell, p}}
$$


for $i \in\{1,2,3, \ell, 4,5,6\}$. We shall henceforth set $m_{i}=m_{i, p}$ and $n_{j}=n_{j, p}$, for convenience. It clearly follows from (7.4) and (7.5) that

$$
\begin{aligned}
& m_{1}+m_{2}+m_{3}+m_{4}+m_{5} \in\{0,1\}, \\
& \min \left\{n_{1}, m_{2}+m_{\ell}+m_{4}+m_{5}\right\}=0, \\
& \min \left\{n_{2}, m_{1}+m_{3}\right\}=\min \left\{n_{\ell}, m_{4}+m_{5}+m_{6}\right\}=0,
\end{aligned}
$$

and once combined with (7.1), it is easy to deduce the further relation

$$
\min \left\{m_{6}, n_{1}, n_{2}\right\}=0 .
$$

We now construct a map $\Phi: \mathcal{T}_{1} \rightarrow \mathcal{T}_{2}$. This is obtained via $\Phi:(\boldsymbol{\xi}, \boldsymbol{\tau}) \mapsto\left(\xi_{1}^{\prime}, \ldots, \xi_{6}^{\prime}, \tau_{1}^{\prime}, \tau_{2}^{\prime}, \tau_{\ell}^{\prime}\right)=$ $\left(\boldsymbol{\xi}^{\prime}, \boldsymbol{\tau}^{\prime}\right)$, where

$$
\xi_{i}^{\prime}=\prod_{p} p^{m_{i, p}^{\prime}}, \quad \tau_{1}^{\prime}= \pm \prod_{p} p^{n_{1, p}^{\prime}}, \quad \tau_{2}^{\prime}=\prod_{p} p^{n_{2, p}^{\prime}}, \quad \tau_{\ell}^{\prime}= \pm \prod_{p} p^{n_{\ell, p}^{\prime}}
$$

for suitable values of $m_{i}^{\prime}=m_{i, p}^{\prime}$ and $n_{j}^{\prime}=n_{j, p}^{\prime}$. The idea is to transfer powers of primes $p$ between $\xi_{6}$ and the variables appearing in the final monomial $\tau_{1}^{3} \xi_{1}^{2} \xi_{3}$ of (7.1). We shall set $m_{i}^{\prime}=m_{i}$ for $i \in\{2, \ell, 4,5\}$, and $n_{j}^{\prime}=n_{j}$ for $j \in\{2, \ell\}$. The remaining values of $m_{i}^{\prime}, n_{j}^{\prime}$ are determined in the following way, in which $k$ denotes an arbitrary non-negative integer.

- If $n_{1}=2 k+1, m_{6} \geqslant k+1, m_{3}=0$, then

$$
\left(m_{1}^{\prime}, m_{3}^{\prime}, m_{6}^{\prime}, n_{1}^{\prime}\right)=\left(m_{1}+3 k+1,1, m_{6}-k-1, n_{1}-2 k-1\right) .
$$

- If $n_{1}=2 k+1, m_{6} \geqslant k+1, m_{3}=1$, or if $n_{1}>2 k, m_{6}=k, m_{3}=1$, then

$$
\left(m_{1}^{\prime}, m_{3}^{\prime}, m_{6}^{\prime}, n_{1}^{\prime}\right)=\left(m_{1}+3 k+2,0, m_{6}-k, n_{1}-2 k-1\right) .
$$

- If $n_{1}>2 k, m_{6}=k, m_{3}=0$, or if $n_{1}=2 k, m_{6} \geqslant k$, then

$$
\left(m_{1}^{\prime}, m_{3}^{\prime}, m_{6}^{\prime}, n_{1}^{\prime}\right)=\left(m_{1}+3 k, m_{3}, m_{6}-k, n_{1}-2 k\right) .
$$

We note that the five possibilities detailed here are exhaustive. Furthermore, on recalling the conditions (7.8) and (7.9), it follows from these definitions that

$$
\begin{aligned}
& m_{2}^{\prime}+m_{3}^{\prime}+m_{4}^{\prime}+m_{5}^{\prime} \in\{0,1\}, \quad \min \left\{m_{1}^{\prime}, m_{2}^{\prime}\right\}=0, \\
& \min \left\{n_{1}^{\prime}, m_{2}^{\prime}+m_{3}^{\prime}+m_{\ell}^{\prime}+m_{4}^{\prime}+m_{5}^{\prime}+m_{6}^{\prime}\right\}=0, \\
& \min \left\{n_{2}^{\prime}, m_{1}^{\prime}+m_{3}^{\prime}\right\}=\min \left\{n_{\ell}^{\prime}, m_{4}^{\prime}+m_{5}^{\prime}+m_{6}^{\prime}\right\}=0,
\end{aligned}
$$

and so $\boldsymbol{\xi}^{\prime}, \boldsymbol{\tau}^{\prime}$ satisfy the coprimality relations (7.5), (7.6) and (7.7). Finally, it is easily checked that $\left(\boldsymbol{\xi}^{\prime}, \boldsymbol{\tau}^{\prime}\right) \in \mathcal{T}_{2}$.

Conversely, let $\left(\boldsymbol{\xi}^{\prime}, \boldsymbol{\tau}^{\prime}\right) \in \mathcal{T}_{2}$. Then we construct a map $\Phi^{-1}: \mathcal{T}_{2} \rightarrow \mathcal{T}_{1}$, given by $\Phi^{-1}:\left(\boldsymbol{\xi}^{\prime}, \boldsymbol{\tau}^{\prime}\right) \mapsto(\boldsymbol{\xi}, \boldsymbol{\tau})$, by setting $m_{i}=m_{i}^{\prime}$ for $i \in\{2, \ell, 4,5\}$, and $n_{j}=n_{j}^{\prime}$ for $j \in\{2, \ell\}$. The remaining values of $m_{i}, n_{j}$ depend on the value of $m_{1}^{\prime}$ modulo 3 , and are determined in the following way, in which $k$ again denotes an arbitrary non-negative integer.

- If $m_{1}^{\prime} \in\{3 k+1,3 k+2\}$ and $m_{3}^{\prime}=1$, then

$$
\left(m_{1}, m_{3}, m_{6}, n_{1}\right)=\left(m_{1}^{\prime}-3 k-1,0, m_{6}^{\prime}+k+1, n_{1}^{\prime}+2 k+1\right) .
$$

$4^{\mathrm{e}}$ SÉRIE - TOME $40-2007-\mathrm{N}^{\circ} 1$ 
- If $m_{1}^{\prime}=3 k+2$ and $m_{3}^{\prime}=0$, then

$$
\left(m_{1}, m_{3}, m_{6}, n_{1}\right)=\left(m_{1}^{\prime}-3 k-2,1, m_{6}^{\prime}+k, n_{1}^{\prime}+2 k+1\right) .
$$

- If $m_{1}^{\prime}=3 k+1$ and $m_{3}^{\prime}=0$, or if $m_{1}^{\prime}=3 k$, then

$$
\left(m_{1}, m_{3}, m_{6}, n_{1}\right)=\left(m_{1}^{\prime}-3 k, m_{3}^{\prime}, m_{6}^{\prime}+k, n_{1}^{\prime}+2 k\right) .
$$

Again it is straightforward to check that $(\boldsymbol{\xi}, \boldsymbol{\tau}) \in \mathcal{T}_{1}$.

Finally one verifies that $\Phi \Phi^{-1}=\mathrm{id}_{\mathcal{T}_{2}}$ and $\Phi^{-1} \Phi=\mathrm{id}_{\mathcal{T}_{1}}$, and that the points $(\boldsymbol{\xi}, \boldsymbol{\tau}) \in \mathcal{T}_{1}$ and $\left(\boldsymbol{\xi}^{\prime}, \boldsymbol{\tau}^{\prime}\right) \in \mathcal{T}_{2}$ correspond to the same point in $\mathcal{E}$ under the map $\Psi$. This therefore completes the proof of Lemma 14.

We are now ready to relate the quantity $\# \mathcal{E}(B)$, as given by (6.1), to an appropriate count on the universal torsor. Now it is clear that under the substitutions (7.2), the height restriction $|\mathbf{x}| \leqslant B$ in $\mathcal{E}(B)$ lifts to

$$
\max \left\{\xi^{(1,2,2,0,1,2,3)} \tau_{2},\left|\tau_{\ell}\right|, \xi^{(2,3,4,3,4,5,6)}, \xi^{(2,2,3,1,2,3,4)}\left|\tau_{1}\right|\right\} \leqslant B,
$$

for $(\boldsymbol{\xi}, \boldsymbol{\tau}) \in \mathcal{T}_{2}$.

It will be convenient to introduce the set

$$
\mathcal{F}:=\left\{\boldsymbol{\xi} \in \mathbb{N}^{7}:\left|\mu\left(\xi_{2} \xi_{3} \xi_{4} \xi_{5}\right)\right|=1, \operatorname{gcd}\left(\xi_{1}, \xi_{2} \xi_{\ell} \xi_{4} \xi_{5}\right)=\operatorname{gcd}\left(\xi_{\ell}, \xi_{2} \xi_{3}\right)=1\right\} .
$$

It is easy to check that $(\boldsymbol{\xi}, \boldsymbol{\tau}) \in \mathcal{T}_{2}$ if and only if $(\boldsymbol{\xi}, \boldsymbol{\tau}) \in \mathcal{F} \times \mathbb{Z}_{*} \times \mathbb{N} \times \mathbb{Z}_{*}$ and satisfies (7.1), (7.5), and (7.7). With this new notation in mind, it therefore follows from Lemma 14 that

$$
\# \mathcal{E}(B)=\#\left\{(\boldsymbol{\xi}, \boldsymbol{\tau}) \in \mathcal{F} \times \mathbb{Z}_{*} \times \mathbb{N} \times \mathbb{Z}_{*}: \text { (7.1), (7.5), (7.7), (7.10) hold }\right\}
$$

Our final task in this section will be to analyse the inequalities (7.10) in the light of (7.1), to see precisely what conditions they force upon the variables $\boldsymbol{\xi}, \tau$. It is at this point of the argument that the real-valued functions introduced in $\S 5$ enter the picture.

Let $\boldsymbol{\xi} \in \mathbb{N}^{7}$. Then it will be convenient to define

$$
\alpha:=B^{-1 / 2} \xi_{1} \xi_{2}^{3 / 2} \xi_{3}^{2} \xi_{\ell}^{3 / 2} \xi_{4}^{2} \xi_{5}^{5 / 2} \xi_{6}^{3},
$$

and

$$
X_{1}:=B^{1 / 3} \xi_{1}^{-2 / 3} \xi_{3}^{-1 / 3} \xi_{\ell} \xi_{4}^{2 / 3} \xi_{5}^{1 / 3}, \quad X_{2}:=B^{1 / 2} \xi_{2}^{-1 / 2} \xi_{\ell}^{3 / 2} \xi_{4} \xi_{5}^{1 / 2}
$$

Now let $(\boldsymbol{\xi}, \boldsymbol{\tau}) \in \mathcal{T}_{2}$ such that (7.10) holds. Then it immediately follows from (7.10) that

$$
0 \leqslant \alpha \leqslant 1 \text {. }
$$

Recall the definition (5.1) of the function $g_{1}:[0,1] \rightarrow \mathbb{R}$. Then it is not hard to combine (7.1) and (7.10) to conclude that for fixed $\boldsymbol{\xi}$, the variable $\tau_{1}$ is constrained to lie in the interval

$$
X_{1} g_{1}(\alpha) \leqslant \tau_{1} \leqslant X_{1} .
$$

Next we recall the definitions (5.2) and (5.3) of the functions $g_{21}, g_{22}$ that are both defined on $(-\infty, 1] \times[0,1]$. For convenience, we suppose $B \in \mathbb{R} \backslash \overline{\mathbb{Q}}$ is such that for all $\tau_{1}<-X_{1}$ we have

$$
X_{2} g_{21}\left(\tau_{1} / X_{1}, \alpha\right)=X_{2} \sqrt{-1-\left(\tau_{1} / X_{1}\right)^{3}} \notin \mathbb{Z}
$$


Then one deduces from (7.1) and (7.10) that for fixed $\boldsymbol{\xi}, \tau_{1}$, the variable $\tau_{2}$ lies in the interval

$$
X_{2} g_{21}\left(\tau_{1} / X_{1}, \alpha\right)<\tau_{2} \leqslant X_{2} g_{22}\left(\tau_{1} / X_{1}, \alpha\right) .
$$

Putting all of this together, and noting that we automatically have $\left|\tau_{\ell}\right| \leqslant B$ when (7.1) and (7.14)(7.16) are satisfied, we have therefore established the following result.

LEMMA 15. - Let $B \in \mathbb{R} \backslash \overline{\mathbb{Q}}$. Then we have

$$
\# \mathcal{E}(B)=\#\left\{(\boldsymbol{\xi}, \boldsymbol{\tau}) \in \mathcal{F} \times \mathbb{Z}_{*} \times \mathbb{N} \times \mathbb{Z}_{*}: \text { (7.1), (7.5), (7.7), (7.14)-(7.16) hold }\right\}
$$

\section{Proof of Theorem 1: summations}

The goal of this section is to produce a preliminary estimate for $\# \mathcal{E}(B)$. The first step, in $\S 8.1$, will involve summing over the possible values of $\tau_{2}, \tau_{\ell}$, for fixed $\boldsymbol{\xi}, \tau_{1}$. In doing so we shall take care of the summation over $\tau_{\ell}$ by viewing (7.1) as a congruence

$$
\tau_{2}^{2} \xi_{2}+\tau_{1}^{3} \xi_{1}^{2} \xi_{3} \equiv 0\left(\bmod \xi_{\ell}^{3} \xi_{4}^{2} \xi_{5}\right) .
$$

Of course we must first carry out a Möbius inversion in order to account for the coprimality condition $\operatorname{gcd}\left(\tau_{\ell}, \xi_{4} \xi_{5} \xi_{6}\right)=1$, but this presents a purely technical difficulty. The most challenging aspect involves giving a satisfactory treatment of the error term that arises from approximating the number of solutions $\tau_{2}$ to this congruence by an appropriate real-valued function. To facilitate digestion of the text, the handling of the error term will be carried out separately in $\S 8.2$, and this can be omitted at a first reading. It should be remarked that it is mainly for this part of the proof of Theorem 1 that the contents of $\S 3$ and $\S 4$ are necessary.

Next in $\S 8.3$ we shall sum over the possible values of $\tau_{1}$, for fixed $\boldsymbol{\xi}$. Here again there is significant work to be done in handling the overall contribution from the error term. In fact, rather than showing that the overall error term makes a negligible contribution to $\# \mathcal{E}(B)$, we find here that the contribution from this error term produces a highly non-trivial secondary contribution. In view of the technical difficulties involved we have therefore found it convenient to cordon off the said treatment into $\$ 8.4$. This part can also be omitted at a first reading. Finally we shall sum over the remaining variables $\boldsymbol{\xi}$ in $\S 8.5$.

\subsection{Summation over $\tau_{2}$ and $\tau_{\ell}$}

Recall the notations (7.11)-(7.13) introduced above, and let $\left(\boldsymbol{\xi}, \tau_{1}\right) \in \mathcal{F} \times \mathbb{Z}_{*}$ be such that (7.7), (7.14) and (7.15) hold. Then the aim of this section is to estimate the number $N=N\left(\boldsymbol{\xi}, \tau_{1}\right)$ of $\left(\tau_{2}, \tau_{\ell}\right) \in \mathbb{N} \times \mathbb{Z}_{*}$ such that (7.5), (7.16) and the torsor equation (7.1) are fulfilled. Now a Möbius inversion yields

$$
N=\sum_{k_{\ell} \mid \xi_{4} \xi_{5} \xi_{6}} \mu\left(k_{\ell}\right) N_{k_{\ell}}
$$

where $N_{k_{\ell}}$ has the same definition as $N$ except that the coprimality relation $\operatorname{gcd}\left(\tau_{\ell}, \xi_{4} \xi_{5} \xi_{6}\right)=1$ is removed, and (7.1) is replaced by

$$
k_{\ell} \tau_{\ell} \xi_{\ell}^{3} \xi_{4}^{2} \xi_{5}+\tau_{2}^{2} \xi_{2}+\tau_{1}^{3} \xi_{1}^{2} \xi_{3}=0
$$

Let us write $I_{2}$ for the interval (7.16), and define

$$
q:=k_{\ell} \xi_{\ell}^{3} \xi_{4}^{2} \xi_{5}
$$

$4^{\text {e }}$ SÉRIE - TOME $40-2007-\mathrm{N}^{\circ} 1$ 
It now follows that

$$
N_{k_{\ell}}=\#\left\{\tau_{2} \in I_{2} \cap \mathbb{N}: \operatorname{gcd}\left(\tau_{2}, \xi_{1} \xi_{3}\right)=1,-\tau_{2}^{2} \xi_{2} \equiv \tau_{1}^{3} \xi_{1}^{2} \xi_{3}(\bmod q)\right\}
$$

which we henceforth assume to be non-zero. Note that

$$
\operatorname{gcd}\left(\tau_{1}^{3} \xi_{1}^{2} \xi_{3}, q\right)=\operatorname{gcd}\left(\tau_{1}^{3} \xi_{1}^{2} \xi_{3}, \xi_{\ell}^{3} \xi_{4}^{3} \xi_{5}^{2} \xi_{6}, \tau_{2}^{2} \xi_{2}\right)=1
$$

for any $k_{\ell} \mid \xi_{4} \xi_{5} \xi_{6}$. It follows from this that

$$
\operatorname{gcd}\left(\xi_{1} \xi_{2} \xi_{3}, k_{\ell}\right)=1
$$

Since $k_{\ell} \mid q$, we must therefore take care to sum only over values of $k_{\ell} \mid \xi_{4} \xi_{5} \xi_{6}$ such that $\operatorname{gcd}\left(k_{\ell}, \tau_{1} \xi_{1} \xi_{2} \xi_{3}\right)=1$, in order to ensure a non-zero value of $N_{k_{\ell}}$. This latter condition is equivalent to $\operatorname{gcd}\left(k_{\ell}, \xi_{1} \xi_{2} \xi_{3}\right)=1$, for any $k_{\ell} \mid \xi_{4} \xi_{5} \xi_{6}$, since $\tau_{1}$ is coprime to $\xi_{\ell} \xi_{4} \xi_{5} \xi_{6}$.

It is now clear that there exists a unique integer $\varrho$ satisfying $1 \leqslant \varrho \leqslant q$ and $\operatorname{gcd}(\varrho, q)=1$, such that

$$
\tau_{2} \equiv \varrho \tau_{1} \xi_{1}(\bmod q), \quad-\varrho^{2} \xi_{2} \equiv \tau_{1} \xi_{3}(\bmod q)
$$

Thus we have

$$
N=\sum_{\begin{array}{c}
k_{\ell} \mid \xi_{4} \xi_{5} \xi_{6} \\
\operatorname{gcd}\left(k_{\ell}, \xi_{1} \xi_{2} \xi_{3}\right)=1
\end{array}} \mu\left(k_{\ell}\right) \sum_{\substack{1 \leqslant \varrho \leqslant q, \operatorname{gcd}(\varrho, q)=1 \\
-\varrho^{2} \xi_{2} \equiv \tau_{1} \xi_{3}(\bmod q)}} N_{k_{\ell}}(\varrho)
$$

where

$$
\begin{aligned}
N_{k_{\ell}}(\varrho) & =\#\left\{\tau_{2} \in I_{2} \cap \mathbb{N}: \operatorname{gcd}\left(\tau_{2}, \xi_{1} \xi_{3}\right)=1, \tau_{2} \equiv \varrho \tau_{1} \xi_{1}(\bmod q)\right\} \\
& =\sum_{k_{2} \mid \xi_{1} \xi_{3}} \mu\left(k_{2}\right) \#\left\{\tau_{2} \in\left(k_{2}^{-1} I_{2}\right) \cap \mathbb{N}: k_{2} \tau_{2} \equiv \varrho \tau_{1} \xi_{1}(\bmod q)\right\}
\end{aligned}
$$

and in which we also know that $\operatorname{gcd}\left(k_{2}, q\right) \leqslant \operatorname{gcd}\left(\xi_{1} \xi_{3}, q\right)=1$. The summand here is just the number of positive integers restricted to lie in a certain interval, that lie in a fixed residue class modulo $q$. When we come in the next section to sum the error term that arises from estimating this quantity over all of the remaining variables, a significant awkwardness is caused by the fact that the interval $I_{2}$, as given by (7.16), depends intimately upon $\tau_{1}$. Write $\bar{k}_{2}$ for the multiplicative inverse of $k_{2}$ modulo $q$. Then it is straightforward to deduce from Lemma 7 that

$$
\begin{aligned}
N_{k_{\ell}}(\varrho) & =\sum_{k_{2} \mid \xi_{1} \xi_{3}} \mu\left(k_{2}\right)\left(\frac{h_{2}\left(\tau_{1}\right)-h_{1}\left(\tau_{1}\right)}{q}+r\left(h_{1}\left(\tau_{1}\right), h_{2}\left(\tau_{1}\right) ; \bar{k}_{2} \varrho \tau_{1} \xi_{1}, q\right)\right) \\
& =X_{2} g_{2}\left(\tau_{1} / X_{1}, \alpha\right) \frac{\phi^{*}\left(\xi_{1} \xi_{3}\right)}{q}+\sum_{k_{2} \mid \xi_{1} \xi_{3}} \mu\left(k_{2}\right) r\left(h_{1}\left(\tau_{1}\right), h_{2}\left(\tau_{1}\right) ; \bar{k}_{2} \varrho \tau_{1} \xi_{1}, q\right),
\end{aligned}
$$

where we have set

$$
h_{i}(x):=\frac{X_{2} g_{2 i}\left(x / X_{1}, \alpha\right)}{k_{2}},
$$

for $i=1,2$, and where $g_{2}$ is given by (5.4) and (5.5), and $\phi^{*}$ is given by (4.3). On recalling the definition (8.1) of $q$, we have therefore established the following result for fixed $\left(\boldsymbol{\xi}, \tau_{1}\right) \in \mathcal{F} \times \mathbb{Z}_{*}$ such that (7.7), (7.14) and (7.15) hold. 
LEMMA 16. - We have

$$
N\left(\boldsymbol{\xi}, \tau_{1}\right)=\frac{X_{2}}{\xi_{\ell}^{3} \xi_{4}^{2} \xi_{5}} g_{2}\left(\tau_{1} / X_{1}, \alpha\right) \Sigma\left(\boldsymbol{\xi}, \tau_{1}\right)+E_{1}\left(\boldsymbol{\xi}, \tau_{1}\right)
$$

where

$$
\Sigma\left(\boldsymbol{\xi}, \tau_{1}\right):=\phi^{*}\left(\xi_{1} \xi_{3}\right) \sum_{\begin{array}{c}
k_{\ell} \mid \xi_{4} \xi_{5} \xi_{6} \\
\operatorname{gcd}\left(k_{\ell}, \xi_{1} \xi_{2} \xi_{3}\right)=1
\end{array}} \frac{\mu\left(k_{\ell}\right)}{k_{\ell}} \sum_{\substack{1 \leqslant \varrho \leqslant q, \operatorname{gcd}(\varrho, q)=1 \\
-\varrho^{2} \xi_{2} \equiv \tau_{1} \xi_{3}(\bmod q)}} 1
$$

and

$$
\begin{aligned}
& E_{1}\left(\boldsymbol{\xi}, \tau_{1}\right):= \sum_{\begin{array}{c}
k_{\ell} \mid \xi_{4} \xi_{5} \xi_{6} \\
\operatorname{gcd}\left(k_{\ell}, \xi_{1} \xi_{2} \xi_{3}\right)=1
\end{array}} \sum_{k_{2} \mid \xi_{1} \xi_{3}} \mu\left(k_{2}\right) \mu\left(k_{\ell}\right) \\
& \sum_{\substack{1 \leqslant \varrho \leqslant q, \operatorname{gcd}(\varrho, q)=1 \\
-\varrho^{2} \xi_{2} \equiv \tau_{1} \xi_{3}(\bmod q)}} r\left(h_{1}\left(\tau_{1}\right), h_{2}\left(\tau_{1}\right) ; \bar{k}_{2} \varrho \tau_{1} \xi_{1}, q\right) .
\end{aligned}
$$

For any $T \geqslant 1$, define the pair of sets

$$
\begin{aligned}
& \mathcal{A}(B, T):=\left\{\boldsymbol{\xi} \in \mathcal{F}: 0 \leqslant \alpha \leqslant 1, \xi_{\ell}^{3} \xi_{4}^{2} \xi_{5} \geqslant T\right\}, \\
& \mathcal{B}(B, T):=\left\{\boldsymbol{\xi} \in \mathcal{F}: 0 \leqslant \alpha \leqslant 1, \xi_{\ell}^{3} \xi_{4}^{2} \xi_{5}<T\right\},
\end{aligned}
$$

where $\mathcal{F}$ is given by (7.11) and $\alpha$ is given by (7.12). As indicated above, the hardest task that we shall face is showing that $E_{1}\left(\boldsymbol{\xi}, \tau_{1}\right)$ makes a satisfactory contribution to $\# \mathcal{E}(B)$, once summed over all of the relevant values of $\boldsymbol{\xi}, \tau_{1}$. That this is so is recorded in the following result, the proof of which is postponed until the following section.

PROPOSITION 1. - Let $\varepsilon>0$. Then we have

$$
\sum_{\boldsymbol{\xi} \in \mathcal{A}(B, 1)} \sum_{\substack{g_{1}(\alpha) \leqslant \tau_{1} / X_{1} \leqslant 1 \\ \operatorname{gcd}\left(\tau_{1}, \xi_{2} \xi_{3} \xi_{\ell} \xi_{4} \xi_{5} \xi_{6}\right)=1}} E_{1}\left(\boldsymbol{\xi}, \tau_{1}\right) \ll_{\varepsilon} B^{43 / 48+\varepsilon} .
$$

\subsection{Proof of Proposition 1}

Recall the definition of $r\left(t_{1}, t_{2} ; a, q\right)$ from the statement of Lemma 7, and the definitions (7.12), (8.3) of $\alpha$ and $h_{i}$. Then it will suffice to estimate

$$
\begin{aligned}
& Z_{i}(B):= \sum_{\boldsymbol{\xi} \in \mathcal{A}(B, 1)} \sum_{\substack{k_{\ell} \mid \xi_{4} \xi_{5} \xi_{6} \\
\operatorname{gcd}\left(k_{\ell}, \xi_{1} \xi_{2} \xi_{3}\right)=1}} \sum_{k_{2} \mid \xi_{1} \xi_{3}} \mu\left(k_{2}\right) \mu\left(k_{\ell}\right) \\
& \sum_{\substack{g_{1}(\alpha) \leqslant \tau_{1} / X_{1} \leqslant 1 \\
\operatorname{gcd}\left(\tau_{1}, \xi_{2} \xi_{3} \xi_{6}\right)=1}} \sum_{\substack{1 \leqslant \varrho \leqslant q, \operatorname{gcd}(\varrho, q)=1 \\
-\varrho^{2} \xi_{2} \equiv \tau_{1} \xi_{3}(\bmod q)}} \psi\left(\frac{h_{i}\left(\tau_{1}\right)-\bar{k}_{2} \xi_{1} \varrho \tau_{1}}{q}\right),
\end{aligned}
$$

for $i=1,2$. Here, we have been able to replace the coprimality condition $\operatorname{gcd}\left(\tau_{1}, \xi_{2} \xi_{3} \xi_{\ell} \xi_{4} \xi_{5} \xi_{6}\right)=1$ in (7.7), by $\operatorname{gcd}\left(\tau_{1}, \xi_{2} \xi_{3} \xi_{6}\right)=1$. Write $Z(B)=Z_{1}(B)-Z_{2}(B)$. Then our goal is to establish the following result, since this clearly suffices for the proof of Proposition 1.

$4^{\text {e }}$ SÉRIE - TOME $40-2007-\mathrm{N}^{\circ} 1$ 
LEMMA 17. - Let $\varepsilon>0$. Then we have

$$
Z(B)=\mathrm{O}_{\varepsilon}\left(B^{43 / 48+\varepsilon}\right) .
$$

Before commencing the proof of Lemma 17 proper, it will be convenient to introduce some more notation. Let

$$
q_{0}:=\xi_{\ell}^{3} \xi_{4}^{2} \xi_{5}
$$

Then $q=k_{\ell} q_{0}$ by (8.1), and

$$
X_{1}=\frac{q_{0}^{1 / 3} B^{1 / 3}}{\xi_{1}^{2 / 3} \xi_{3}^{1 / 3}}, \quad X_{2}=\frac{q_{0}{ }^{1 / 2} B^{1 / 2}}{\xi_{2}^{1 / 2}},
$$

by (7.13). It clearly follows that

$$
\frac{X_{2}}{\xi_{1} \xi_{3} X_{1}}=\frac{q_{0}{ }^{1 / 6} B^{1 / 6}}{\xi_{1}^{1 / 3} \xi_{2}^{1 / 2} \xi_{3}^{2 / 3}} \geqslant 1,
$$

for any $\boldsymbol{\xi} \in \mathbb{N}^{7}$ such that $0 \leqslant \alpha \leqslant 1$. We also define

$$
Y_{1}:=\frac{X_{1}}{k_{1}}=\frac{q_{0}{ }^{1 / 3} B^{1 / 3}}{k_{1} \xi_{1}^{2 / 3} \xi_{3}^{1 / 3}}, \quad Y_{2}:=\frac{X_{2}}{k_{2}}=\frac{q_{0}{ }^{1 / 2} B^{1 / 2}}{k_{2} \xi_{2}^{1 / 2}}
$$

for any $k_{1}, k_{2} \in \mathbb{N}$, and set

$$
f_{i}(x):=Y_{2} g_{2 i}\left(x / Y_{1}, \alpha\right)
$$

for $i=1,2$.

We begin the proof of Lemma 17 by applying a Möbius inversion to remove the coprimality condition $\operatorname{gcd}\left(\tau_{1}, \xi_{2} \xi_{3} \xi_{6}\right)=1$ from the summation over $\tau_{1}$. Thus we obtain

$$
\begin{gathered}
Z_{i}(B)=\sum_{\boldsymbol{\xi} \in \mathcal{A}(B, 1)} \sum_{\substack{k_{\ell} \mid \xi_{4} \xi_{5} \xi_{6} \\
\operatorname{gcd}\left(k_{\ell}, \xi_{1} \xi_{2} \xi_{3}\right)=1}} \sum_{\substack{k_{2} \mid \xi_{1} \xi_{3} \\
\xi_{3}}} \sum_{\substack{k_{1} \mid \xi_{2} \xi_{3} \xi_{6} \\
\operatorname{gcd}\left(k_{1}, q\right)=1}} \mu\left(k_{1}\right) \mu\left(k_{2}\right) \mu\left(k_{\ell}\right) \\
\sum_{\substack{g_{1}(\alpha) \leqslant \tau_{1} / Y_{1} \leqslant 1 \\
\operatorname{gcd}\left(\tau_{1}, q\right)=1}} \psi\left(\frac{f_{i}\left(\tau_{1}\right)-k_{1} \bar{k}_{2} \xi_{1} \varrho \tau_{1}}{q}\right), \\
-\varrho^{2} \xi_{2} \equiv k_{1} \tau_{1} \xi_{3}(\bmod q)
\end{gathered}
$$

for $i=1,2$, where $f_{1}, f_{2}$ are given above. Here we have inserted the necessary coprimality conditions $\operatorname{gcd}\left(k_{1}, q\right)=1$ and $\operatorname{gcd}\left(\tau_{1}, q\right)=1$, and then removed reference to the condition $\operatorname{gcd}(\varrho, q)=1$ in the summation over $\varrho$, since this must automatically hold.

Let $I \subset(-\infty, 1]$ be an interval and let $f$ be a real-valued function on $I$. Then on setting

$$
Q_{I}(f):=S_{I}\left(f, q ;-\xi_{2}, k_{1} \xi_{3}, k_{1} \bar{k}_{2} \xi_{1}\right),
$$

in the notation of (4.1), we therefore conclude that

$$
\left|Z_{i}(B)\right| \leqslant \sum_{\boldsymbol{\xi} \in \mathcal{A}(B, 1)} \sum_{\begin{array}{c}
k_{\ell} \mid \xi_{4} \xi_{5} \xi_{6} \\
\operatorname{gcd}\left(k_{\ell}, \xi_{1} \xi_{2} \xi_{3}\right)=1
\end{array}} \sum_{k_{2} \mid \xi_{1} \xi_{3}} \sum_{\begin{array}{c}
k_{1} \mid \xi_{2} \xi_{3} \xi_{6} \\
\operatorname{gcd}\left(k_{1}, q\right)=1
\end{array}}\left|Q_{\left[g_{1}(\alpha) Y_{1}, Y_{1}\right]}\left(f_{i}\right)\right|
$$


for $i=1,2$. Since $\operatorname{gcd}\left(k_{1} \bar{k}_{2} \xi_{1} \xi_{2} \xi_{3}, q\right)=1$, the path is now clear to try and apply Lemmas 9,10 and 11 to estimate the right-hand side of (8.10).

We recall the definition (8.4) of $\mathcal{A}(B, T)$ and $\mathcal{B}(B, T)$. Then for $i=1,2$, we let $U_{i}(B, T)$ denote the overall contribution to the right-hand side of (8.10) arising from summing the $\boldsymbol{\xi}$ over the set $\mathcal{A}(B, T)$, and we let $V_{i}(B, T)$ denote the corresponding contribution from the set $\mathcal{B}(B, T)$. We will then have

$$
|Z(B)| \leqslant U_{1}(B, T)+U_{2}(B, T)+V_{1}(B, T)+V_{2}(B, T),
$$

and it will remain to choose a suitable value of $T \geqslant 1$ that minimises the bound. The hardest of the quantities to estimate is $U_{i}(B, T)$, for $i=1,2$, and it is these two sums that we consider first. It is likely that some small improvement is possible in the exponents of these results. However we have chosen not to pursue this here, since what we have achieved is sufficient for our purposes.

LEMMA 18. - Let $\varepsilon>0$. Then for any $T \geqslant \sqrt{B}$ we have

$$
U_{1}(B, T) \ll_{\varepsilon} B^{43 / 48+\varepsilon}+\frac{B^{3 / 2+\varepsilon}}{T^{10 / 9}} .
$$

Proof. - The thrust of the proof is concerned with estimating $Q_{\left[g_{1}(\alpha) Y_{1}, Y_{1}\right]}\left(f_{1}\right)$. In fact it will be necessary to break the interval $\left[g_{1}(\alpha) Y_{1}, Y_{1}\right]$ into a disjoint union of smaller intervals, on which we can apply Lemmas 10 and 11 most effectively. This reflects the fact that for certain values of $x \in\left[g_{1}(\alpha) Y_{1}, Y_{1}\right]$ the functions $f_{1}^{\prime}(x)$ and $f_{1}^{\prime \prime}(x)$ can be abnormally large or small. Let

$$
\delta=\delta(B), \quad \eta=\eta(B),
$$

be certain positive functions of the parameter $B$, such that $\delta>\eta$. These functions will be selected in due course, but we remark at the outset that $\delta<B^{-1 / 1000}$. We now write $\left[g_{1}(\alpha) Y_{1}, Y_{1}\right]=$ $\bigcup_{i=1}^{7} I_{i}$, with

$$
\begin{aligned}
& I_{1}:=\left[g_{1}(\alpha) Y_{1},-\left(2^{2 / 3}+\eta^{3 / 4}\right) Y_{1}\right], \\
& I_{2}:=\left(-\left(2^{2 / 3}+\eta^{3 / 4}\right) Y_{1},-\left(2^{2 / 3}-\eta^{3 / 4}\right) Y_{1}\right], \\
& I_{3}:=\left(-\left(2^{2 / 3}-\eta^{3 / 4}\right) Y_{1},-\frac{2^{2 / 3}+1}{2} Y_{1}\right], \\
& I_{4}:=\left(-\frac{2^{2 / 3}+1}{2} Y_{1},-(1+\delta) Y_{1}\right], \\
& I_{5}:=\left(-(1+\delta) Y_{1},-(1+\eta) Y_{1}\right], \\
& I_{6}:=\left(-(1+\eta) Y_{1},-Y_{1}\right], \\
& I_{7}:=\left(-Y_{1}, Y_{1}\right] .
\end{aligned}
$$

These intervals are clearly all non-empty, for sufficiently large values of $B$, and it is not hard to see that

$$
\begin{array}{ll}
\operatorname{meas}\left(I_{1}\right) \ll Y_{1} / \alpha^{2 / 3}, & \operatorname{meas}\left(I_{2}\right)=2 \eta^{3 / 4} Y_{1}, \\
\operatorname{meas}\left(I_{3}\right) \ll Y_{1}, & \operatorname{meas}\left(I_{4}\right) \ll Y_{1}, \\
\operatorname{meas}\left(I_{5}\right) \ll \delta Y_{1}, & \operatorname{meas}\left(I_{6}\right)=\eta Y_{1}, \\
\operatorname{meas}\left(I_{7}\right)=2 Y_{1} . &
\end{array}
$$

$4^{\text {e }}$ SÉRIE - TOME $40-2007-\mathrm{N}^{\circ} 1$ 
Here we have used the definition (5.1) of $g_{1}$, which shows in particular that $g_{1}$ is negative, to deduce that

$$
g_{1}(\alpha) \ll \alpha^{-2 / 3}
$$

Before proceeding with the task of estimating $Q_{i}:=Q_{I_{i}}\left(f_{1}\right)$ for $1 \leqslant i \leqslant 7$, we first deduce from (5.2) and (8.9) that

$$
f_{1}(x)= \begin{cases}Y_{2} \sqrt{-1-\left(x / Y_{1}\right)^{3}}, & x \in I_{1} \cup \cdots \cup I_{6}, \\ 0, & x \in I_{7}\end{cases}
$$

In particular it follows that on the interior of each interval $I_{i}$, the real-valued function $f_{1}$ is infinitely differentiable, with $f_{1}^{\prime}$ being monotonic and of constant sign.

Moreover, we see that

$$
\left|\int_{I_{i}} f_{1}^{\prime}(t) \mathrm{d} t\right|+1 \ll \lambda_{0}^{(i)}
$$

for $1 \leqslant i \leqslant 7$, with

$$
\begin{array}{lll}
\lambda_{0}^{(1)}=Y_{2} / \alpha, & \lambda_{0}^{(2)}=\eta^{3 / 4} Y_{2}+1, & \lambda_{0}^{(3)}=\lambda_{0}^{(4)}=Y_{2}, \\
\lambda_{0}^{(5)}=\delta^{1 / 2} Y_{2}+1, & \lambda_{0}^{(6)}=\eta^{1 / 2} Y_{2}+1, & \lambda_{0}^{(7)}=1 .
\end{array}
$$

It follows from these remarks, together with (3.6), that $f_{1} \in C^{1}\left(I_{i} ; A \lambda_{0}^{(i)}\right)$ for $1 \leqslant i \leqslant 7$, for some absolute constant $A>0$.

We begin by using Lemma 10 to estimate $Q_{2}, Q_{6}$ and $Q_{7}$. Thus it follows from the trivial bounds $Y_{1} \leqslant X_{1}$ and $Y_{2} \leqslant X_{2}$, that

$$
\sum_{i=2,6,7}\left|Q_{i}\right| \ll_{\varepsilon} q^{\varepsilon} X_{1}^{\varepsilon}\left(q^{1 / 2}+\frac{\eta^{3 / 4} X_{1}^{1 / 2} X_{2}^{1 / 2}}{q^{1 / 4}}+\frac{X_{1}+X_{2}^{1 / 2}}{q^{1 / 4}}\right) .
$$

Turning to the size of $Q_{1}, Q_{3}, Q_{4}$ and $Q_{5}$, we must examine in more detail the behaviour of the function $f_{1}$ on $I_{1} \cup I_{3} \cup I_{4} \cup I_{5}$. Now it is clear that

$$
f_{1}^{\prime}(x)=\frac{-3 Y_{2}\left(x / Y_{1}\right)^{2}}{2 Y_{1} \sqrt{-1-\left(x / Y_{1}\right)^{3}}}, \quad f_{1}^{\prime \prime}(x)=\frac{3 Y_{2}\left(x / Y_{1}\right)\left(4+\left(x / Y_{1}\right)^{3}\right)}{4 Y_{1}^{2}\left(-1-\left(x / Y_{1}\right)^{3}\right)^{3 / 2}} .
$$

In particular $f_{1}^{\prime}$ has constant sign on $I_{1} \cup I_{3} \cup I_{4} \cup I_{5}$, and is monotonic increasing (resp. decreasing) on $I_{1}$ (resp. on $I_{3} \cup I_{4} \cup I_{5}$ ). We therefore deduce from (8.13) that

$$
f_{1}^{\prime}(x) \ll \begin{cases}\alpha^{-4 / 3} Y_{2} / Y_{1}, & x \in I_{1}, \\ Y_{2} / Y_{1}, & x \in I_{3}, \\ \delta^{-1 / 2} Y_{2} / Y_{1}, & x \in I_{4}, \\ \eta^{-1 / 2} Y_{2} / Y_{1}, & x \in I_{5} .\end{cases}
$$

Moreover, it is clear from (8.7) and (8.8) that

$$
Y_{2} / Y_{1} \geqslant X_{2} /\left(\xi_{1} \xi_{3} X_{1}\right) \geqslant 1
$$

ANNALES SCIENTIFIQUES DE L'ÉCOLE NORMALE SUPÉRIEURE 
On $\left(-\infty,-Y_{1}\right]$ it is clear that the function $\left|f_{1}^{\prime \prime}(x)\right|$ is minimised at $x=-2^{2 / 3} Y_{1}$, where it takes the value 0 . Hence on $I_{1} \cup I_{3}$ we have

$$
\eta^{3 / 4} Y_{2} / Y_{1}^{2} \ll\left|f_{1}^{\prime \prime}(x)\right| \ll Y_{2} / Y_{1}^{2},
$$

on $I_{4}$ we have

$$
Y_{2} / Y_{1}^{2} \ll\left|f_{1}^{\prime \prime}(x)\right| \ll \delta^{-3 / 2} Y_{2} / Y_{1}^{2}
$$

and finally on $I_{5}$ we have

$$
\delta^{-3 / 2} Y_{2} / Y_{1}^{2} \ll\left|f_{1}^{\prime \prime}(x)\right| \ll \eta^{-3 / 2} Y_{2} / Y_{1}^{2} .
$$

On recalling (8.15), we have therefore shown that there exists an absolute constant $A>0$ such that $f_{1}$ belongs to the sets

$$
\begin{aligned}
& C^{2}\left(I_{1} ; A \lambda_{0}^{(1)}, \frac{A Y_{2}}{\alpha^{4 / 3} Y_{1}}, \frac{c_{1} \eta^{3 / 4} Y_{2}}{Y_{1}^{2}}, \frac{1}{\eta^{3 / 4}}\right), \quad C^{2}\left(I_{3} ; A \lambda_{0}^{(3)}, \frac{A Y_{2}}{Y_{1}}, \frac{c_{3} \eta^{3 / 4} Y_{2}}{Y_{1}^{2}}, \frac{1}{\eta^{3 / 4}}\right), \\
& C^{2}\left(I_{4} ; A \lambda_{0}^{(4)}, \frac{A Y_{2}}{\delta^{1 / 2} Y_{1}}, \frac{c_{4} Y_{2}}{Y_{1}^{2}}, \frac{1}{\delta^{3 / 2}}\right), \quad C^{2}\left(I_{5} ; A \lambda_{0}^{(5)}, \frac{A Y_{2}}{\eta^{1 / 2} Y_{1}}, \frac{c_{5} Y_{2}}{\delta^{3 / 2} Y_{1}^{2}}, \frac{\delta^{3 / 2}}{\eta^{3 / 2}}\right),
\end{aligned}
$$

for appropriate absolute constants $c_{1}, c_{3}, c_{4}, c_{5}>0$. Let us write

$$
f_{1} \in C^{2}\left(I_{i} ; A \lambda_{0}^{(i)}, \lambda_{1}^{(i)}, \lambda_{2}^{(i)}, j^{(i)}\right)
$$

for $i=1,3,4,5$, and recall the estimates (8.12) for meas $\left(I_{i}\right)$. We are now ready to complete our estimates for $Q_{1}, Q_{3}, Q_{4}, Q_{5}$ via an application of Lemma 11. Thus we obtain

$$
\begin{aligned}
& Q_{1} \ll_{\varepsilon} q^{\varepsilon} Y_{1}^{\varepsilon}\left(q^{1 / 2}+\frac{Y_{1}}{\alpha^{2 / 3} q^{1 / 3}}+\frac{Y_{1}^{1 / 3} Y_{2}^{1 / 3}}{\alpha^{10 / 9} \eta^{1 / 4}}+\frac{\eta^{1 / 2} Y_{1}^{1 / 3} Y_{2}^{4 / 3}}{\alpha^{13 / 9} q^{3 / 2}}+\frac{Y_{2}}{\alpha^{4 / 3} q}\right), \\
& Q_{3} \ll_{\varepsilon} q^{\varepsilon} Y_{1}^{\varepsilon}\left(q^{1 / 2}+\frac{Y_{1}}{q^{1 / 3}}+\frac{Y_{1}^{1 / 3} Y_{2}^{1 / 3}}{\eta^{1 / 4}}+\frac{\eta^{1 / 2} Y_{1}^{1 / 3} Y_{2}^{4 / 3}}{q^{3 / 2}}+\frac{Y_{2}}{q}\right), \\
& Q_{4} \ll_{\varepsilon} q^{\varepsilon} Y_{1}^{\varepsilon}\left(q^{1 / 2}+\frac{Y_{1}}{q^{1 / 3}}+\frac{Y_{1}^{1 / 3} Y_{2}^{1 / 3}}{\delta^{1 / 3}}+\frac{\delta^{1 / 6} Y_{1}^{1 / 3} Y_{2}^{4 / 3}}{q^{3 / 2}}+\frac{Y_{2}}{\delta^{3 / 2} q}\right),
\end{aligned}
$$

and

$$
Q_{5} \ll_{\varepsilon} q^{\varepsilon} Y_{1}^{\varepsilon}\left(q^{1 / 2}+\frac{\delta Y_{1}}{q^{1 / 3}}+\frac{\delta^{5 / 6} Y_{1}^{1 / 3} Y_{2}^{1 / 3}}{\eta^{1 / 3}}+\frac{\delta^{5 / 6} \eta^{1 / 6} Y_{1}^{1 / 3} Y_{2}^{4 / 3}}{q^{3 / 2}}+\frac{\delta^{2} Y_{2}}{\eta^{3 / 2} q}\right) .
$$

It turns out that we shall need to minimise the third terms in these estimates for $Q_{1}, Q_{3}, Q_{4}$ and $Q_{5}$. We therefore make the selection

$$
\delta=\eta^{2 / 7}
$$

so that in particular $\delta>\eta$. With this choice we may conclude that

$$
\sum_{i=1,3,4,5}\left|Q_{i}\right| \ll_{\varepsilon} q^{\varepsilon} X_{1}^{\varepsilon}\left(q^{1 / 2}+\frac{X_{1}}{\alpha^{2 / 3} q^{1 / 3}}+\frac{X_{1}^{1 / 3} X_{2}^{1 / 3}}{\alpha^{10 / 9} \eta^{1 / 4}}+\frac{X_{1}^{1 / 3} X_{2}^{4 / 3}}{\alpha^{13 / 9} q^{3 / 2}}+\frac{X_{2}}{\alpha^{4 / 3} q}+\frac{X_{2}}{\eta q}\right),
$$

since $Y_{1} \leqslant X_{1}, Y_{2} \leqslant X_{2}$ and $\alpha, \eta \leqslant 1$.

$4^{\text {e }}$ SÉRIE - TOME $40-2007-\mathrm{N}^{\circ} 1$ 
Write $J$ for the interval $\left[g_{1}(\alpha) Y_{1}, Y_{1}\right]$. Then (8.16) and (8.17) provide us with an overall estimate for $Q_{J}\left(f_{1}\right)$. This estimate is made somewhat complicated by the large number of terms that it contains, and so we proceed to consider the overall contribution to the right-hand side of (8.10) from some of the individual terms. Let us begin by handling the term $q^{1 / 2}$ that appears in both (8.16) and (8.17). From (8.1) and the inequality $k_{\ell} \leqslant \xi_{4} \xi_{5} \xi_{6}$ it follows that $q \leqslant \xi_{\ell}^{3} \xi_{4}^{3} \xi_{5}^{2} \xi_{6}$. Thus we see that

$$
\begin{aligned}
\sum_{\xi_{1}^{2} \xi_{2}^{3} \xi_{3}^{4} \xi_{\ell}^{3} \xi_{4}^{4} \xi_{5}^{5} \xi_{6}^{6} \leqslant B} q^{1 / 2} & \leqslant \sum_{\xi_{1}^{2} \xi_{2}^{3} \xi_{3}^{4} \xi_{\ell}^{3} \xi_{4}^{4} \xi_{5}^{5} \xi_{6}^{6} \leqslant B} \xi_{\ell}^{3 / 2} \xi_{4}^{3 / 2} \xi_{5} \xi_{6}^{1 / 2} \\
& \leqslant \sum_{\xi_{2}^{3} \xi_{3}^{4} \xi_{\ell}^{3} \xi_{4}^{4} \xi_{5}^{5} \xi_{6}^{6} \leqslant B} \frac{B^{1 / 2}}{\xi_{2}^{3 / 2} \xi_{3}^{2} \xi_{4}^{1 / 2} \xi_{5}^{3 / 2} \xi_{6}^{5 / 2}} \ll B^{5 / 6},
\end{aligned}
$$

where the summations are over all $\xi \in \mathbb{N}^{7}$ in the prescribed range. Next we recall the definitions (7.12), (8.5), (8.6) of $\alpha, q_{0}$ and $X_{1}, X_{2}$, together with the bounds $0 \leqslant \alpha \leqslant 1$ and $q \geqslant q_{0} \geqslant T$ that hold for any $\boldsymbol{\xi} \in \mathcal{A}(B, T)$. Then it follows that

$$
\alpha=B^{-1 / 2} \xi_{1} \xi_{2}^{3 / 2} \xi_{3}^{2} \xi_{4} \xi_{5}^{2} \xi_{6}^{3} q_{0}{ }^{1 / 2},
$$

whence

$$
\max \left\{\frac{X_{1}}{q^{1 / 4}}, \frac{X_{1}}{\alpha^{2 / 3} q^{1 / 3}}\right\} \leqslant \frac{X_{1}}{\alpha^{2 / 3} q_{0}{ }^{1 / 4}} \leqslant \frac{B^{2 / 3}}{\xi_{1}^{4 / 3} \xi_{2} \xi_{3}^{5 / 3} \xi_{\ell}^{3 / 4} \xi_{4}^{7 / 6} \xi_{5}^{19 / 12} \xi_{6}^{2}} .
$$

Thus we have

$$
\sum_{\xi_{1}^{2} \xi_{2}^{3} \xi_{3}^{4} \xi_{\ell}^{3} \xi_{4}^{4} \xi_{5}^{5} \xi_{6}^{6} \leqslant B} \max \left\{\frac{X_{1}}{q^{1 / 4}}, \frac{X_{1}}{\alpha^{2 / 3} q^{1 / 3}}\right\} \ll \sum_{\xi_{2} \xi_{\ell} \leqslant B^{1 / 3}} \frac{B^{2 / 3}}{\xi_{2} \xi_{\ell}^{3 / 4}} \ll B^{3 / 4} .
$$

Similarly one notes that

$$
\max \left\{\frac{X_{2}^{1 / 2}}{q^{1 / 4}}, \frac{X_{2}}{\alpha^{4 / 3} q}, \frac{X_{2}}{\eta q}\right\} \leqslant \frac{B^{1 / 4}}{\xi_{2}^{1 / 4}}+\frac{B^{1 / 2}}{\eta \xi_{2}^{1 / 2} q_{0}^{1 / 2}}+\frac{B^{7 / 6}}{\xi_{1}^{4 / 3} \xi_{2}^{5 / 2} \xi_{3}^{8 / 3} \xi_{\ell} \xi_{4}^{2} \xi_{5}^{3} \xi_{6}^{4} q_{0}^{2 / 3}},
$$

whence

$$
\sum_{\xi_{1}^{2} \xi_{2}^{3} \xi_{3}^{4} \xi_{\ell}^{3} \xi_{4}^{4} \xi_{5}^{5} \xi_{6}^{6} \leqslant B} \max \left\{\frac{X_{2}^{1 / 2}}{q^{1 / 4}}, \frac{X_{2}}{\alpha^{4 / 3} q}, \frac{X_{2}}{\eta q}\right\} \ll \frac{B^{3 / 4}}{\eta}+B^{5 / 6} \log B .
$$

Here we have used the fact that $q_{0} \geqslant T \geqslant \sqrt{B}$. Now let us treat the term

$$
\frac{X_{1}^{1 / 3} X_{2}^{4 / 3}}{\alpha^{\theta} q^{3 / 2}} \leqslant \frac{B^{7 / 9+\theta / 2}}{\xi_{\ell}\left(\xi_{1} \xi_{2} \xi_{3} \xi_{4} \xi_{5} \xi_{6}\right)^{\theta} q_{0}^{7 / 18+\theta / 2}},
$$

for any $\theta>1$. But for any such $\theta$ we see that

$$
\sum_{\xi_{1}^{2} \xi_{2}^{3} \xi_{3}^{4} \xi_{\ell}^{3} \xi_{4}^{4} \xi_{5}^{\xi} \xi_{6}^{6} \leqslant B} \frac{X_{1}^{1 / 3} X_{2}^{4 / 3}}{\alpha^{\theta} q^{3 / 2}} \ll \frac{B^{7 / 9+\theta / 2} \log B}{T^{7 / 18+\theta / 2}}
$$

since $q_{0} \geqslant T$. 
It turns out that for the choice of $\eta$ and $T$ that we shall make, all of the terms (8.18)(8.21) make a negligible contribution in our final estimate for $U_{1}(B, T)$. There are in effect two dominant contributions, the first of which is the term

$$
\frac{\eta^{3 / 4} X_{1}^{1 / 2} X_{2}^{1 / 2}}{q^{1 / 4}} \leqslant \frac{\eta^{3 / 4} q_{0}{ }^{1 / 6} B^{5 / 12}}{\xi_{1}^{1 / 3} \xi_{2}^{1 / 4} \xi_{3}^{1 / 6}}=\frac{\eta^{3 / 4} B^{5 / 12} \xi_{\ell}^{1 / 2} \xi_{4}^{1 / 3} \xi_{5}^{1 / 6}}{\xi_{1}^{1 / 3} \xi_{2}^{1 / 4} \xi_{3}^{1 / 6}}
$$

in (8.16), which therefore produces a contribution

$$
\sum_{\xi_{1}^{2} \xi_{2}^{3} \xi_{3}^{4} \xi_{\ell}^{3} \xi_{4}^{4} \xi_{5}^{5} \xi_{6}^{6} \leqslant B} \frac{\eta^{3 / 4} X_{1}^{1 / 2} X_{2}^{1 / 2}}{q^{1 / 4}} \ll \eta^{3 / 4} B^{11 / 12} .
$$

The second major contribution comes from the term

$$
\frac{X_{1}^{1 / 3} X_{2}^{1 / 3}}{\alpha^{10 / 9} \eta^{1 / 4}} \leqslant \frac{B^{5 / 6}}{\eta^{1 / 4} \xi_{\ell}^{5 / 6}\left(\xi_{1} \xi_{2} \xi_{3} \xi_{4} \xi_{5} \xi_{6}\right)^{10 / 9}},
$$

in (8.17), which plainly yields the contribution

$$
\sum_{\xi_{1}^{2} \xi_{2}^{3} \xi_{3}^{4} \xi_{\ell}^{3} \xi_{4}^{4} \xi_{5}^{5} \xi_{6}^{6} \leqslant B} \frac{X_{1}^{1 / 3} X_{2}^{1 / 3}}{\alpha^{10 / 9} \eta^{1 / 4}} \ll \frac{B^{8 / 9}}{\eta^{1 / 4}} .
$$

Bringing together (8.18)-(8.23) in the right-hand side of (8.10), we may therefore conclude that

$$
U_{1}(B, T) \ll_{\varepsilon} \eta^{3 / 4} B^{11 / 12+\varepsilon}+\frac{B^{8 / 9+\varepsilon}}{\eta^{1 / 4}}+\frac{B^{3 / 4+\varepsilon}}{\eta}+\frac{B^{3 / 2+\varepsilon}}{T^{10 / 9}},
$$

for any $\varepsilon>0$. The first two terms represent the dominant contribution, and so it remains to choose a value of $\eta$ that balances them. The proof of Lemma 18 is thus completed by taking $\eta=B^{-1 / 36}$.

LEMMA 19. - Let $\varepsilon>0$. Then for any $T \geqslant \sqrt{B}$ we have

$$
U_{2}(B, T) \ll_{\varepsilon} B^{59 / 66+\varepsilon}+\frac{B^{5 / 3+\varepsilon}}{T^{23 / 18}}
$$

Proof. - The proof of this result is very similar to that of the previous lemma, and so we shall be brief. Now for any $\alpha>0$ it is easy to see that

$$
g_{1}(\alpha) \leqslant-\left(1 / \alpha^{2}-1\right)^{1 / 3}
$$

As above it will be necessary to break the interval $\left[g_{1}(\alpha) Y_{1}, Y_{1}\right]$ into a disjoint union of smaller intervals, in order to estimate $Q_{\left[g_{1}(\alpha) Y_{1}, Y_{1}\right]}\left(f_{2}\right)$ most effectively. Let $\eta$ be a small positive real number. This will be selected in due course, but it may be assumed that $\eta<B^{-1 / 1000}$. Thus we write $\left[g_{1}(\alpha) Y_{1}, Y_{1}\right]=\bigcup_{i=1}^{5} I_{i}$, with 


$$
\begin{aligned}
& I_{1}:=\left[g_{1}(\alpha) Y_{1},-\left(1 / \alpha^{2}-1\right)^{1 / 3} Y_{1}\right], \\
& I_{2}:=\left(-\left(1 / \alpha^{2}-1\right)^{1 / 3} Y_{1},-\eta Y_{1}\right], \\
& I_{3}:=\left(-\eta Y_{1}, \eta Y_{1}\right], \\
& I_{4}:=\left(\eta Y_{1},\left(1-\eta^{8 / 3}\right) Y_{1}\right], \\
& I_{5}:=\left(\left(1-\eta^{8 / 3}\right) Y_{1}, Y_{1}\right] .
\end{aligned}
$$

Observe that

$$
\begin{aligned}
& \operatorname{meas}\left(I_{1}\right) \ll Y_{1}, \quad \operatorname{meas}\left(I_{2}\right) \ll Y_{1} / \alpha^{2 / 3}, \quad \operatorname{meas}\left(I_{3}\right)=2 \eta Y_{1}, \\
& \operatorname{meas}\left(I_{4}\right) \ll Y_{1}, \quad \operatorname{meas}\left(I_{5}\right)=\eta^{8 / 3} Y_{1} \text {. }
\end{aligned}
$$

Before proceeding with the task of estimating $Q_{i}:=Q_{I_{i}}\left(f_{2}\right)$ for $1 \leqslant i \leqslant 5$, we first deduce from (5.3) and (8.9) that

$$
f_{2}(x)= \begin{cases}\alpha^{-1} Y_{2}, & x \in I_{1} \\ Y_{2} \sqrt{1-\left(x / Y_{1}\right)^{3}}, & x \in I_{2} \cup I_{3} \cup I_{4} \cup I_{5}\end{cases}
$$

In particular it follows that on each interval $I_{i}$, the real-valued function $f_{2}$ is infinitely differentiable, with $f_{2}^{\prime}$ being monotonic and of constant sign. Moreover,

$$
\left|\int_{I_{i}} f_{2}^{\prime}(t) \mathrm{d} t\right|+1 \ll \lambda_{0}^{(i)}
$$

for $1 \leqslant i \leqslant 5$, with

$$
\begin{array}{ll}
\lambda_{0}^{(1)}=1, & \lambda_{0}^{(2)}=Y_{2} / \alpha, \quad \lambda_{0}^{(3)}=\eta^{3} Y_{2}+1, \\
\lambda_{0}^{(4)}=Y_{2}, & \lambda_{0}^{(5)}=\eta^{4 / 3} Y_{2}+1 .
\end{array}
$$

Hence $f_{2} \in C^{1}\left(I_{i} ; A \lambda_{0}^{(i)}\right)$ for $1 \leqslant i \leqslant 5$, for an absolute constant $A>0$.

We begin by using Lemma 10 to estimate $Q_{1}, Q_{3}$ and $Q_{5}$. Thus it follows from (8.24) and (8.25) that

$$
\sum_{i=1,3,5}\left|Q_{i}\right| \ll_{\varepsilon} q^{\varepsilon} Y_{1}^{\varepsilon}\left(q^{1 / 2}+\frac{Y_{1}}{q^{1 / 3}}+\frac{\eta^{2} Y_{1}^{1 / 2} Y_{2}^{1 / 2}}{q^{1 / 4}}+\frac{Y_{2}^{1 / 2}}{q^{1 / 4}}\right) .
$$

Turning to the size of $Q_{2}$ and $Q_{4}$, we must examine the behaviour of $f_{2}$ on $I_{2} \cup I_{4}$. Now it is clear that

$$
f_{2}^{\prime}(x)=\frac{-3 Y_{2}\left(x / Y_{1}\right)^{2}}{2 Y_{1} \sqrt{1-\left(x / Y_{1}\right)^{3}}}, \quad f_{2}^{\prime \prime}(x)=\frac{-3 Y_{2}\left(x / Y_{1}\right)\left(\left(x / Y_{1}\right)^{3}-4\right)}{4 Y_{1}^{2}\left(1-\left(x / Y_{1}\right)^{3}\right)^{3 / 2}} .
$$

In particular $f_{2}^{\prime}$ has constant sign on $I_{2}$, and $\left|f_{2}^{\prime}\right|$ is monotonic decreasing (resp. increasing) on $I_{2}$ (resp. on $I_{4}$ ). We therefore deduce that

$$
f_{2}^{\prime}(x) \ll \begin{cases}\alpha^{-1 / 3} Y_{2} / Y_{1}, & x \in I_{2}, \\ \eta^{-4 / 3} Y_{2} / Y_{1}, & x \in I_{4}\end{cases}
$$

ANNALES SCIENTIFIQUES DE L'ÉCOLE NORMALE SUPÉRIEURE 
Next we note that on $\left(-\infty, Y_{1}\right]$ the function $\left|f_{1}^{\prime \prime}(x)\right|$ is minimised at $x=0$, where it takes the value 0 . Hence we have

$$
\eta Y_{2} / Y_{1}^{2} \ll\left|f_{2}^{\prime \prime}(x)\right| \ll \begin{cases}Y_{2} / Y_{1}^{2}, & \text { if } x \in I_{2}, \\ \eta^{-4} Y_{2} / Y_{1}^{2}, & \text { if } x \in I_{4}\end{cases}
$$

We have therefore shown that there exists an absolute constant $A>0$ such that

$$
f_{2} \in C^{2}\left(I_{2} ; A \lambda_{0}^{(2)}, \frac{A Y_{2}}{\alpha^{1 / 3} Y_{1}}, \frac{c_{2} \eta Y_{2}}{Y_{1}^{2}}, \frac{1}{\eta}\right), \quad f_{2} \in C^{2}\left(I_{4} ; A \lambda_{0}^{(4)}, \frac{A Y_{2}}{\eta^{4 / 3} Y_{1}}, \frac{c_{4} \eta Y_{2}}{Y_{1}^{2}}, \frac{1}{\eta^{5}}\right),
$$

for appropriate absolute constants $c_{2}, c_{4}>0$. Let us write

$$
f_{2} \in C^{2}\left(I_{i} ; A \lambda_{0}^{(i)}, \lambda_{1}^{(i)}, \lambda_{2}^{(i)}, j^{(i)}\right)
$$

for $i=2,4$. On recalling (8.24), we may therefore deduce from Lemma 11 that

$$
Q_{2} \ll_{\varepsilon} q^{\varepsilon} Y_{1}^{\varepsilon}\left(q^{1 / 2}+\frac{Y_{1}}{\alpha^{2 / 3} q^{1 / 3}}+\frac{Y_{1}^{1 / 3} Y_{2}^{1 / 3}}{\alpha^{4 / 9} \eta^{1 / 3}}+\frac{\eta^{2 / 3} Y_{1}^{1 / 3} Y_{2}^{4 / 3}}{\alpha^{16 / 9} q^{3 / 2}}+\frac{Y_{2}}{\alpha^{4 / 3} q}\right),
$$

and

$$
Q_{4} \ll_{\varepsilon} q^{\varepsilon} Y_{1}^{\varepsilon}\left(q^{1 / 2}+\frac{Y_{1}}{q^{1 / 3}}+\frac{Y_{1}^{1 / 3} Y_{2}^{1 / 3}}{\eta^{4 / 9}}+\frac{\eta^{10 / 9} Y_{1}^{1 / 3} Y_{2}^{4 / 3}}{q^{3 / 2}}+\frac{Y_{2}}{\eta^{4} q}\right) .
$$

Write $J$ for the interval $\left[g_{1}(\alpha) Y_{1}, Y_{1}\right]$. Then the estimates (8.26)-(8.28) together provide us with an overall estimate for $Q_{J}\left(f_{2}\right)$. Once taken into the right-hand side of (8.10), the summation over $\boldsymbol{\xi}$ being only over $\boldsymbol{\xi} \in \mathcal{A}(B, T)$, we may draw upon the proof of Lemma 18 to handle the contribution from all of the individual terms. Thus it follows from (8.18)-(8.20) that

$$
\sum_{\boldsymbol{\xi} \in \mathcal{A}(B, T)}\left(q^{1 / 2}+\frac{X_{1}}{\alpha^{2 / 3} q^{1 / 3}}+\frac{X_{2}^{1 / 2}}{q^{1 / 4}}+\frac{X_{2}}{\alpha^{4 / 3} q}+\frac{X_{2}}{\eta^{4} q}\right) \ll B^{5 / 6} \log B+\frac{B^{3 / 4}}{\eta^{4}}
$$

since $T \geqslant \sqrt{B}$. Similarly, (8.21) gives

$$
\sum_{\boldsymbol{\xi} \in \mathcal{A}(B, T)} \frac{X_{1}^{1 / 3} X_{2}^{4 / 3}}{\alpha^{16 / 9} q^{3 / 2}} \ll \frac{B^{5 / 3} \log B}{T^{23 / 18}} .
$$

These will all make a negligible contribution in our final estimate for the quantity $U_{2}(B, T)$. There are now three dominant contributions, the first of which is the term $\eta^{2} X_{1}^{1 / 2} X_{2}^{1 / 2} / q^{1 / 4}$ in (8.26). This produces an overall contribution

$$
\sum_{\boldsymbol{\xi} \in \mathcal{A}(B, T)} \frac{\eta^{2} X_{1}^{1 / 2} X_{2}^{1 / 2}}{q^{1 / 4}} \ll \eta^{2} B^{11 / 12},
$$

by (8.22). Next we note that

$$
\max \left\{\frac{X_{1}^{1 / 3} X_{2}^{1 / 3}}{\alpha^{4 / 9} \eta^{1 / 3}}, \frac{X_{1}^{1 / 3} X_{2}^{1 / 3}}{\eta^{4 / 9}}\right\} \leqslant \frac{X_{1}^{1 / 3} X_{2}^{1 / 3}}{\alpha^{4 / 9} \eta^{4 / 9}} \leqslant \frac{q_{0}^{1 / 18} B^{1 / 2}}{\eta^{4 / 9} \xi_{1}^{2 / 3} \xi_{2}^{5 / 6} \xi_{3} \xi_{4}^{4 / 9} \xi_{5}^{8 / 9} \xi_{6}^{4 / 3}} .
$$

$4^{e}$ SÉRIE - TOME $40-2007-\mathrm{N}^{\circ} 1$ 
Hence we may argue as for (8.23) to deduce that the second major contribution is

$$
\sum_{\boldsymbol{\xi} \in \mathcal{A}(B, T)}\left(\frac{X_{1}^{1 / 3} X_{2}^{1 / 3}}{\alpha^{4 / 9} \eta^{1 / 3}}+\frac{X_{1}^{1 / 3} X_{2}^{1 / 3}}{\eta^{4 / 9}}\right) \ll \frac{B^{8 / 9}}{\eta^{4 / 9}} .
$$

We may now bring together (8.29)-(8.32) in the right-hand side of (8.10), in order to conclude that

$$
U_{2}(B, T) \ll_{\varepsilon} \eta^{2} B^{11 / 12+\varepsilon}+\frac{B^{8 / 9+\varepsilon}}{\eta^{4 / 9}}+\frac{B^{3 / 4+\varepsilon}}{\eta^{4}}+\frac{B^{5 / 3+\varepsilon}}{T^{23 / 18}},
$$

for any $\varepsilon>0$. As in the proof of Lemma 18, the first two terms represent the dominant contribution and we therefore select $\eta=B^{-1 / 88}$. This completes the proof of Lemma 19 .

Our final task is to estimate the sizes of $V_{1}(B, T)$ and $V_{2}(B, T)$. This is entirely straightforward, since it will suffice just to employ a trivial upper bound for the sums $Q_{\left[g_{1}(\alpha) Y_{1}, Y_{1}\right]}\left(f_{1}\right)$ and $Q_{\left[g_{1}(\alpha) Y_{1}, Y_{1}\right]}\left(f_{2}\right)$. Thus it follows from (8.13) and Lemma 9 that

$$
Q_{\left[g_{1}(\alpha) Y_{1}, Y_{1}\right]}\left(f_{i}\right) \ll_{\varepsilon} \frac{q^{\varepsilon} Y_{1}}{\alpha^{2 / 3}} \ll_{\varepsilon} \frac{B^{2 / 3+\varepsilon}}{\xi_{1}^{4 / 3} \xi_{2} \xi_{3}^{5 / 3} \xi_{4}^{2 / 3} \xi_{5}^{4 / 3} \xi_{6}^{2}},
$$

on substituting the definitions of $\alpha, Y_{1}$ into this estimate. But by definition of the set $\mathcal{B}(B, T)$, we must have $q_{0}<T$, whence

$$
\xi_{\ell}<\frac{T^{1 / 3}}{\xi_{4}^{2 / 3} \xi_{5}^{1 / 3}} .
$$

On summing over all values of $\boldsymbol{\xi} \in \mathcal{B}(B, T)$ we therefore obtain the following result.

LemmA 20. - Let $\varepsilon>0$ and let $i=1$ or 2 . Then for any $T \geqslant 1$ we have

$$
V_{i}(B, T) \ll_{\varepsilon} T^{1 / 3} B^{2 / 3+\varepsilon} .
$$

We are now in a position to draw together Lemmas 18,19 and 20 in (8.11). Thus we conclude that

$$
Z(B) \ll_{\varepsilon} B^{43 / 48+\varepsilon}+\frac{B^{3 / 2+\varepsilon}}{T^{10 / 9}}+\frac{B^{5 / 3+\varepsilon}}{T^{23 / 18}}+T^{1 / 3} B^{2 / 3+\varepsilon},
$$

for any $\varepsilon>0$ and any $T \geqslant \sqrt{B}$. We therefore complete the proof of Lemma 17, and so the proof of Proposition 1, by taking $T=B^{18 / 29}$.

\subsection{Summation over $\tau_{1}$}

For fixed $\xi \in \mathcal{F}$ such that (7.14) holds, we proceed to sum the main term in Lemma 16 over all $\tau_{1}$ satisfying (7.7) and (7.15). Thus our task is to estimate

$$
N^{\prime}=N^{\prime}(\boldsymbol{\xi}):=\frac{X_{2}}{\xi_{\ell}^{3} \xi_{4}^{2} \xi_{5}} \sum_{\substack{g_{1}(\alpha) \leqslant \tau_{1} / X_{1} \leqslant 1 \\ \operatorname{gcd}\left(\tau_{1}, \xi_{2} \xi_{3} \xi_{\ell} \xi_{4} \xi_{5} \xi_{6}\right)=1}} g_{2}\left(\tau_{1} / X_{1}, \alpha\right) \Sigma\left(\boldsymbol{\xi}, \tau_{1}\right)
$$

where $g_{2}$ is given by (5.4) and $\Sigma\left(\boldsymbol{\xi}, \tau_{1}\right)$ is as in the statement of Lemma 16. 
Let $t_{1}, t_{2} \in \mathbb{R}$ such that $t_{2} \geqslant t_{1}$. Then we begin by deriving an asymptotic formula for

$$
\mathcal{N}\left(t_{1}, t_{2}\right):=\phi^{*}\left(\xi_{1} \xi_{3}\right) \sum_{\begin{array}{c}
k_{\ell} \mid \xi_{4} \xi_{5} \xi_{6} \\
\operatorname{gcd}\left(k_{\ell}, \xi_{1} \xi_{2} \xi_{3}\right)=1
\end{array}} \frac{\mu\left(k_{\ell}\right)}{k_{\ell}} \sum_{\substack{1 \leqslant \varrho \leqslant q \\
\operatorname{gcd}(\varrho, q)=1}} N_{k_{\ell}}^{\prime}\left(\varrho ; t_{1}, t_{2}\right)
$$

where $q$ is given by (8.1) and

$$
N_{k_{\ell}}^{\prime}\left(\varrho ; t_{1}, t_{2}\right)=\#\left\{\tau_{1} \in\left[t_{1}, t_{2}\right]: \operatorname{gcd}\left(\tau_{1}, \xi_{2} \xi_{3} \xi_{\ell} \xi_{4} \xi_{5} \xi_{6}\right)=1,-\varrho^{2} \xi_{2} \equiv \tau_{1} \xi_{3}(\bmod q)\right\} .
$$

In view of the fact that $\operatorname{gcd}\left(\varrho^{2} \xi_{2}, q\right)=1$, we may plainly replace the condition $\operatorname{gcd}\left(\tau_{1}, \xi_{2} \xi_{3} \xi_{\ell} \xi_{4} \xi_{5} \xi_{6}\right)=1$ by $\operatorname{gcd}\left(\tau_{1}, \xi_{2} \xi_{3} \xi_{6}\right)=1$ in the definition of $N_{k_{\ell}}^{\prime}\left(\varrho ; t_{1}, t_{2}\right)$. On performing a further Möbius inversion, we obtain

$$
\mathcal{N}\left(t_{1}, t_{2}\right)=\phi^{*}\left(\xi_{1} \xi_{3}\right) \sum_{\substack{k_{\ell} \mid \xi_{4} \xi_{5} \xi_{6} \\ \operatorname{gcd}\left(k_{\ell}, \xi_{1} \xi_{2} \xi_{3}\right)=1}} \frac{\mu\left(k_{\ell}\right)}{k_{\ell}} \sum_{\substack{k_{1} \mid \xi_{2} \xi_{3} \xi_{6} \\ \operatorname{gcd}\left(k_{1}, q\right)=1}} \mu\left(k_{1}\right) \sum_{\substack{1 \leqslant \varrho \leqslant q \\ \operatorname{gcd}(\varrho, q)=1}} N_{k_{1}, k_{\ell}}^{\prime}\left(\varrho ; t_{1}, t_{2}\right),
$$

where

$$
N_{k_{1}, k_{\ell}}^{\prime}\left(\varrho ; t_{1}, t_{2}\right)=\#\left\{\tau_{1} \in\left[t_{1} / k_{1}, t_{2} / k_{1}\right]:-\varrho^{2} \xi_{2} \equiv k_{1} \tau_{1} \xi_{3}(\bmod q)\right\} .
$$

We have therefore reduced the problem to once again estimating the number of integers which are restricted to lie in a certain interval, and that lie in a fixed residue class modulo $q$.

Let $a=a\left(\boldsymbol{\xi}, k_{1}, k_{\ell}\right)$ be the unique positive integer such that $a \leqslant q$, with $\operatorname{gcd}(a, q)=1$ and

$$
-\xi_{2} \equiv k_{1} a \xi_{3}(\bmod q)
$$

Then $-\varrho^{2} \xi_{2} \equiv k_{1} \tau_{1} \xi_{3}(\bmod q)$ if and only if $\tau_{1} \equiv a \varrho^{2}(\bmod q)$. Using Lemma 7, we therefore conclude that

$$
N_{k_{1}, k_{\ell}}^{\prime}\left(\varrho ; t_{1}, t_{2}\right)=\frac{t_{2}-t_{1}}{k_{1} q}+r\left(t_{1} / k_{1}, t_{2} / k_{1} ; a \varrho^{2}, q\right),
$$

if $t_{1} \notin \mathbb{Z}$. Now let

$$
\vartheta(\boldsymbol{\xi})=\phi^{*}\left(\xi_{2} \xi_{3} \xi_{\ell} \xi_{4} \xi_{5} \xi_{6}\right) \frac{\phi^{*}\left(\xi_{4} \xi_{5} \xi_{6}\right) \phi^{*}\left(\xi_{1} \xi_{3}\right)}{\phi^{*}\left(\operatorname{gcd}\left(\xi_{6}, \xi_{1} \xi_{2} \xi_{3}\right)\right)},
$$

if $\boldsymbol{\xi} \in \mathcal{F}$, and $\vartheta(\boldsymbol{\xi})=0$ otherwise. Then for any $t_{1}, t_{2} \in \mathbb{R}$ such that $t_{2} \geqslant t_{1}$ and $t_{1} \notin \mathbb{Z}$, we have therefore shown that

$$
\mathcal{N}\left(t_{1}, t_{2}\right)=\vartheta(\boldsymbol{\xi})\left(t_{2}-t_{1}\right)+\mathcal{R}\left(t_{1}, t_{2}\right)
$$

in (8.33), where

$$
\mathcal{R}\left(t_{1}, t_{2}\right)=\phi^{*}\left(\xi_{1} \xi_{3}\right) \sum_{\substack{k_{\ell} \mid \xi_{4} \xi_{5} \xi_{6} \\ \operatorname{gcd}\left(k_{\ell}, \xi_{1} \xi_{2} \xi_{3}\right)=1}} \frac{\mu\left(k_{\ell}\right)}{k_{\ell}} \sum_{\substack{k_{1} \mid \xi_{2} \xi_{3} \xi_{6} \\ \operatorname{gcd}\left(k_{1}, q\right)=1}} \mu\left(k_{1}\right) \sum_{\substack{1 \leqslant \varrho \leqslant q \\ \operatorname{gcd}(\varrho, q)=1}} r\left(t_{1} / k_{1}, t_{2} / k_{1} ; a \varrho^{2}, q\right) .
$$

Define the function $g_{3}:[0,1] \rightarrow \mathbb{R}$ on the unit interval, given by

$$
g_{3}(v):=\int_{g_{1}(v)}^{1} g_{2}(u, v) \mathrm{d} u
$$

$4^{\mathrm{e}}$ SÉRIE - TOME $40-2007-\mathrm{N}^{\circ} 1$ 
Then a straightforward application of partial summation yields the following result, in which $D_{1} g_{2}$ is the derivative of $g_{2}$ with respect to the first variable, and $\boldsymbol{\xi} \in \mathcal{F}$ is such that (7.14) holds.

LEMMA 21. - We have

$$
N^{\prime}(\boldsymbol{\xi})=\frac{\vartheta(\boldsymbol{\xi}) X_{1} X_{2}}{\xi_{\ell}^{3} \xi_{4}^{2} \xi_{5}} g_{3}(\alpha)+E_{2}(\boldsymbol{\xi})
$$

with

$$
E_{2}(\boldsymbol{\xi}):=\frac{-X_{2}}{\xi_{\ell}^{3} \xi_{4}^{2} \xi_{5}} \int_{-\alpha^{-4 / 3}}^{1}\left(D_{1} g_{2}\right)(u, \alpha) \mathcal{R}\left(-\alpha^{-4 / 3} X_{1}, X_{1} u\right) \mathrm{d} u .
$$

In the expression for $E_{2}(\boldsymbol{\xi})$ we have used the fact that $g_{2}(u, v)=0$ for $u \geqslant 1$, as follows from (5.4). Much as in the initial calculation of $N\left(\boldsymbol{\xi}, \tau_{1}\right)$, we shall have to work rather hard to handle the overall contribution from the error term $E_{2}(\boldsymbol{\xi})$ in this estimate. Whereas the error term in Lemma 16 ultimately made a negligible final contribution to $\# \mathcal{E}(B)$, we will show that this is no longer the case here.

Proposition 2. - Let $\varepsilon>0$. Then there exists $\beta \in \mathbb{R}$ such that

$$
\sum_{\boldsymbol{\xi} \in \mathcal{A}(B, 1)} E_{2}(\boldsymbol{\xi})=\beta B+\mathrm{O}_{\varepsilon}\left(B^{25 / 28+\varepsilon}\right)
$$

where $\mathcal{A}(B, 1)$ is given by (8.4).

The proof of this result is undertaken in the following section. In particular the explicit value of $\beta$ is given below in (8.50).

\subsection{Proof of Proposition 2}

Throughout this section we shall make frequent use of the inequality $\log |\boldsymbol{\xi}| \ll \log B$, that follows from (7.14). We begin with a rather crude upper bound for $\mathcal{R}\left(t_{1}, t_{2}\right)$, as given by (8.35). Recall the definition of $r\left(t_{1} / k_{1}, t_{2} / k_{1} ; a \varrho^{2}, q\right)$ from Lemma 7, together with that of $q$ from (8.1). Then Lemma 8 yields

$$
\begin{aligned}
\mathcal{R}\left(t_{1}, t_{2}\right) & \ll_{\varepsilon} \phi^{*}\left(\xi_{1} \xi_{3}\right) \sum_{k_{\ell} \mid \xi_{4} \xi_{5} \xi_{6}} \frac{\left|\mu\left(k_{\ell}\right)\right|}{k_{\ell}} \sum_{k_{1} \mid \xi_{2} \xi_{3} \xi_{6}}\left|\mu\left(k_{1}\right)\right| q^{1 / 2+\varepsilon} \\
& \ll_{\varepsilon} 2^{\omega\left(\xi_{4} \xi_{5} \xi_{6}\right)+\omega\left(\xi_{2} \xi_{3} \xi_{6}\right)}\left(\xi_{\ell}^{3} \xi_{4}^{2} \xi_{5}\right)^{1 / 2+\varepsilon} \\
& \ll_{\varepsilon} 4^{\omega\left(\xi_{2} \xi_{3} \xi_{6}\right)}\left(\xi_{\ell}^{3} \xi_{4}^{2} \xi_{5}\right)^{1 / 2+2 \varepsilon}
\end{aligned}
$$

for any $\varepsilon>0$.

Ultimately, our proof of Proposition 2 will involve an initial summation of $E_{2}(\boldsymbol{\xi})$ over the variable $\xi_{1}$. It will therefore be convenient to define

$$
Y_{1}:=\frac{B^{1 / 2}}{\xi^{(0,3 / 2,2,3 / 2,2,5 / 2,3)}}=\frac{\xi_{1}}{\alpha},
$$

where $\alpha$ is given by (7.12). In particular this notation takes the place of that introduced in (8.8), and it is clear that $Y_{1}$ is independent of $\xi_{1}$. Moreover, the inequality $\alpha \leqslant 1$ is plainly equivalent 
to $\xi_{1} \leqslant Y_{1}$. We also set

$$
q_{1}:=\xi^{(0,1,1,2,2,2,2)}, \quad q_{2}:=\xi^{(0,2,2,1,2,3,4)},
$$

and observe that $X_{1}=q_{1} \alpha^{-2 / 3}$ in (7.13). On recalling the definition of $X_{2}$, we therefore conclude that $E_{2}(\boldsymbol{\xi})$ can be rewritten as

$$
E_{2}(\boldsymbol{\xi})=\frac{-B^{1 / 2}}{\xi^{(0,1 / 2,0,3 / 2,1,1 / 2,0)}} \int_{-\alpha^{-4 / 3}}^{1}\left(D_{1} g_{2}\right)(u, \alpha) \mathcal{R}\left(-\alpha^{-2} q_{1}, \alpha^{-2 / 3} q_{1} u\right) \mathrm{d} u
$$

Let $T \geqslant 1$ be a parameter, to be chosen in due course, and recall the definition of $\mathcal{A}(B, T)$ from (8.4). Our first task is to show that we obtain a satisfactory contribution by summing $E_{2}(\boldsymbol{\xi})$ over those values of $\boldsymbol{\xi}$ for which either $q_{2}>T$, or $\xi_{1} \leqslant Y_{1} / T^{1 / 2}$.

LEMMA 22. - Let $\varepsilon>0$. Then for any $T \geqslant 1$ we have

$$
\sum_{\substack{\boldsymbol{\xi} \in \mathcal{A}(B, 1) \\ T \text { or } \xi_{1} \leqslant Y_{1} / T^{1 / 2}}} E_{2}(\boldsymbol{\xi}) \ll_{\varepsilon} B T^{\varepsilon-1 / 2} .
$$

Proof. - On inserting (8.37) into (8.38), and applying the bound (5.7), we obtain

$$
E_{2}(\boldsymbol{\xi}) \ll_{\varepsilon} \frac{B^{1 / 2}}{\xi^{(0,1 / 2,0,3 / 2,1,1 / 2,0)}} 4^{\omega\left(\xi_{2} \xi_{3} \xi_{6}\right)}\left(\xi_{\ell}^{3} \xi_{4}^{2} \xi_{5}\right)^{1 / 2+\varepsilon}
$$

It therefore follows that

$$
\begin{aligned}
\sum_{\xi_{1} \leqslant Y_{1}} E_{2}(\boldsymbol{\xi}) & \ll_{\varepsilon} \frac{4^{\omega\left(\xi_{2} \xi_{3} \xi_{6}\right)}\left(\xi_{\ell}^{3} \xi_{4}^{2} \xi_{5}\right)^{\varepsilon} B}{\xi^{(0,2,2,3 / 2,2,5 / 2,3)}} \\
& \ll_{\varepsilon} \frac{B}{q_{2}{ }^{1 / 2-3 \varepsilon} \xi^{(0,1+\varepsilon, 1+\varepsilon, 1+\varepsilon, 1+\varepsilon, 1+\varepsilon, 1+\varepsilon)}},
\end{aligned}
$$

whence the overall contribution from the case $q_{2}>T$ is $\mathrm{O}_{\varepsilon}\left(B T^{\varepsilon-1 / 2}\right)$, on redefining the choice of $\varepsilon$. This is satisfactory for the lemma.

In the same fashion one deduces from the upper bound (8.39) that

$$
\begin{aligned}
\sum_{\xi_{1} \leqslant Y_{1} / T^{1 / 2}} E_{2}(\boldsymbol{\xi}) & \ll_{\varepsilon} \sum_{\xi_{1} \leqslant Y_{1} / T^{1 / 2}} \frac{4^{\omega\left(\xi_{2} \xi_{3} \xi_{6}\right)}\left(\xi_{\ell}^{3} \xi_{4}^{2} \xi_{5}\right)^{1 / 2+\varepsilon} B^{1 / 2}}{\xi^{(0,1 / 2,0,3 / 2,1,1 / 2,0)}} \\
& \ll_{\varepsilon} \frac{4^{\omega\left(\xi_{2} \xi_{3} \xi_{6}\right)}\left(\xi_{\ell}^{3} \xi_{4}^{2} \xi_{5}\right)^{\varepsilon} B}{T^{1 / 2} \xi^{(0,2,2,3 / 2,2,5 / 2,3)}}
\end{aligned}
$$

and so the overall contribution from the case $\xi_{1} \leqslant Y_{1} / T^{1 / 2}$ is $\mathrm{O}\left(B / T^{1 / 2}\right)$. This too is satisfactory for the lemma.

It is interesting to remark that on taking $T=1$ in Lemma 22, we deduce that the overall contribution from the $E_{2}(\boldsymbol{\xi})$ term in Lemma 21 is $\mathrm{O}(B)$. This is already enough to establish a version of Theorem 1 with the weaker error term $\mathrm{O}(B)$.

In estimating the overall contribution obtained by summing $E_{2}(\xi)$ over all of the relevant values of $\boldsymbol{\xi}$, it henceforth suffices to focus our attention upon those values of $\boldsymbol{\xi}$ for which $q_{2} \leqslant T$ and $\xi_{1}>Y_{1} / T^{1 / 2}$. Let $t_{2} \geqslant t_{1}$ and recall the definition (8.35) of $\mathcal{R}\left(t_{1}, t_{2}\right)$. Then it follows that

$4^{\mathrm{e}}$ SÉRIE - TOME $40-2007-\mathrm{N}^{\circ} 1$ 


$$
\sum_{\substack{\xi_{1} \leqslant Y_{1} \\
\operatorname{gcd}\left(\xi_{1}, \xi_{2} \xi_{\ell} \xi_{4} \xi_{5}\right)=1}} \mathcal{R}\left(t_{1}, t_{2}\right)=\sum_{\substack{k_{\ell} \mid \xi_{4} \xi_{5} \xi_{6} \\
\operatorname{gcd}\left(k_{\ell}, \xi_{2} \xi_{3}\right)=1}} \frac{\mu\left(k_{\ell}\right)}{k_{\ell}} \sum_{\begin{array}{c}
k_{1} \mid \xi_{2} \xi_{3} \xi_{6} \\
\operatorname{gcd}\left(k_{1}, q\right)=1
\end{array}} \mu\left(k_{1}\right) \sum_{\xi_{1} \leqslant Y_{1}} \varpi\left(\xi_{1}\right) \mathcal{R}_{0}\left(t_{1}, t_{2}\right),
$$

with

$$
\varpi\left(\xi_{1}\right):= \begin{cases}\phi^{*}\left(\xi_{1} \xi_{3}\right), & \text { if } \operatorname{gcd}\left(\xi_{1}, q \xi_{2}\right)=1, \\ 0, & \text { otherwise }\end{cases}
$$

and

$$
\mathcal{R}_{0}\left(t_{1}, t_{2}\right):=\sum_{\substack{1 \leqslant \varrho \leqslant q \\ \operatorname{gcd}(\varrho, q)=1}} r\left(t_{1} / k_{1}, t_{2} / k_{1} ; a \varrho^{2}, q\right) .
$$

The function $\mathcal{R}_{0}\left(t_{1}, t_{2}\right)$ is differentiable with respect to $t_{j}$ everywhere outside the discrete set $E$ of integers congruent to $k_{1} a \varrho^{2}$ modulo $q$, for some integer $\varrho$ which is coprime to $q$. It will be useful to record the equalities

$$
\frac{\partial \mathcal{R}_{0}}{\partial t_{j}}\left(t_{1}, t_{2}\right)=(-1)^{j-1} \frac{\phi^{*}(q)}{k_{1}} \quad(j=1,2)
$$

that are valid outside $E$. Moreover, we shall make use of the bound

$$
\mathcal{R}_{0}\left(t_{1}, t_{2}\right) \ll_{\varepsilon} q^{1 / 2+\varepsilon},
$$

that follows immediately from an application of Lemma 8.

Returning to the above calculation, we may clearly combine it with (8.38) to deduce that

$$
\sum_{\substack{Y_{1} / T^{1 / 2}<\xi_{1} \leqslant Y_{1} \\
\operatorname{gcd}\left(\xi_{1}, \xi_{2} \xi_{\ell} \xi_{4} \xi_{5}\right)=1}} E_{2}(\boldsymbol{\xi})=\frac{-B^{1 / 2}}{\xi^{(0,1 / 2,0,3 / 2,1,1 / 2,0)}} \sum_{\begin{array}{c}
k_{\ell} \mid \xi_{4} \xi_{5} \xi_{6} \\
\operatorname{gcd}\left(k_{\ell}, \xi_{2} \xi_{3}\right)=1
\end{array}} \frac{\mu\left(k_{\ell}\right)}{k_{\ell}} \sum_{\substack{k_{1} \mid \xi_{2} \xi_{3} \xi_{6} \\
\operatorname{gcd}\left(k_{1}, q\right)=1}} \mu\left(k_{1}\right)
$$

$$
\sum_{Y_{1} / T^{1 / 2}<\xi_{1} \leqslant Y_{1}} \varpi\left(\xi_{1}\right) G\left(\xi_{1} / Y_{1}\right)
$$

with

$$
G(\alpha):=\int_{g_{1}(\alpha)}^{1}\left(D_{1} g_{2}\right)(u, \alpha) \mathcal{R}_{0}\left(-\alpha^{-2} q_{1}, \alpha^{-2 / 3} q_{1} u\right) \mathrm{d} u .
$$

In particular it follows from (5.7) and (8.41) that

$$
G(\alpha) \ll_{\varepsilon} q^{1 / 2+\varepsilon} .
$$

We shall also need the following complementary result about the size of $G^{\prime}$.

LeMmA 23. - Let $\varepsilon>0$ and let $\alpha \in(0,1]$ such that $\alpha^{2} \neq 2 /(1+\sqrt{5})$. Then

$$
G^{\prime}(\alpha) \ll_{\varepsilon} \frac{q^{1 / 2+\varepsilon}}{\alpha^{2}}+\frac{q_{1}}{k_{1} \alpha^{5 / 3}} .
$$


Proof. - Recall the definitions (5.1), (5.4) of the functions $g_{1}$ and $g_{2}$. When $1+1 / v^{2} \neq 1 / v^{4}$, it is clear that $g_{1}(v)$ is differentiable. Assume therefore that $\alpha^{2} \neq 2 /(1+\sqrt{5})$, with $\alpha \in(0,1]$, and note that the function $\left(D_{2} D_{1} g_{2}\right)(u, v)$ is identically zero.

One can break the integral in (8.43) into a discrete sum of integrals that avoid all of places where the integrand is not differentiable. Combining (5.6) with (8.40), a little thought therefore reveals that

$$
\begin{aligned}
G^{\prime}(\alpha)= & -g_{1}^{\prime}(\alpha)\left(D_{1} g_{2}\right)\left(g_{1}(\alpha), \alpha\right) \mathcal{R}_{0}\left(-q_{1} \alpha^{-2}, q_{1} g_{1}(\alpha) \alpha^{-2 / 3}\right) \\
& -\frac{2 \phi^{*}(q) q_{1} g_{2}\left(g_{1}(\alpha), \alpha\right)}{k_{1} \alpha^{3}}+\frac{2 \phi^{*}(q) q_{1}}{3 \alpha^{5 / 3} k_{1}} \int_{g_{1}(\alpha)}^{1} u\left(D_{1} g_{2}\right)(u, \alpha) \mathrm{d} u .
\end{aligned}
$$

We use (8.41) to estimate the first term, together with the obvious bounds

$$
g_{1}^{\prime}(\alpha) \ll 1 / \alpha^{5 / 3}, \quad\left(D_{1} g_{2}\right)\left(g_{1}(\alpha), \alpha\right) \ll g_{1}(\alpha)^{1 / 2} \ll 1 / \alpha^{1 / 3} .
$$

Thus the first term is easily seen to be $\mathrm{O}_{\varepsilon}\left(q^{1 / 2+\varepsilon} / \alpha^{2}\right)$. Next we deduce from (5.8) that $g_{2}\left(g_{1}(\alpha), \alpha\right) \ll \alpha^{3}$, since here the left-hand side is zero for $\alpha$ sufficiently small and bounded otherwise. The second term in (8.45) is therefore bounded by $\mathrm{O}\left(q_{1} / k_{1}\right)$. Finally we note that it is straightforward to show that the integral in the third term is bounded. Thus the third term in (8.45) is $\mathrm{O}\left(q_{1} /\left(k_{1} \alpha^{5 / 3}\right)\right)$, which completes the proof of Lemma 23.

We now turn to the problem of estimating

$$
S(Y):=\sum_{\xi_{1} \leqslant Y} \varpi\left(\xi_{1}\right)
$$

for any $Y \geqslant 1$, in preparation for an application of integration by parts in (8.42). Let

$$
\phi^{\prime}(n):=\prod_{p \mid n}\left(1+\frac{1}{p}\right)^{-1},
$$

for any $n \in \mathbb{N}$. Then we shall establish the following result rather easily.

LEMmA 24. - We have $S(Y)=S_{P}(Y)+S_{R}(Y)$ for any $Y \geqslant 1$, with

$$
S_{P}(Y)=\frac{6}{\pi^{2}} \phi^{\prime}\left(k_{\ell} \xi_{2} \xi_{3} \xi_{\ell} \xi_{4} \xi_{5}\right) Y, \quad S_{R}(Y)=\mathrm{O}_{\varepsilon}\left(2^{\omega\left(k_{\ell} \xi_{2} \xi_{\ell} \xi_{4} \xi_{5}\right)} Y^{\varepsilon}\right) .
$$

Proof. - To establish the lemma we consider the corresponding Dirichlet series

$$
\begin{aligned}
\sum_{\xi_{1}=1}^{\infty} \frac{\varpi\left(\xi_{1}\right)}{\xi_{1}^{s}} & =\phi^{*}\left(\xi_{3}\right) \prod_{p \nmid k_{\ell} \xi_{2} \xi_{3} \xi_{\ell} \xi_{4} \xi_{5}}\left(1+\frac{1-1 / p}{p^{s}-1}\right) \prod_{p \mid \xi_{3}}\left(\frac{1}{1-p^{-s}}\right) \\
& =\phi^{*}\left(\xi_{3}\right) \zeta(s) \prod_{p \nmid k_{\ell} \xi_{2} \xi_{3} \xi_{\ell} \xi_{4} \xi_{5}}\left(1-\frac{1}{p^{s+1}}\right) \prod_{p \mid k_{\ell} \xi_{2} \xi_{\ell} \xi_{4} \xi_{5}}\left(1-p^{-s}\right),
\end{aligned}
$$

where we have used the relation $\operatorname{gcd}\left(\xi_{3}, k_{\ell}\right)=1$. The result is now immediate on applying Perron's formula.

$4^{\text {e }}$ SÉRIE - TOME $40-2007-\mathrm{N}^{\circ} 1$ 
We are now in good shape to complete the proof of Proposition 2. Recall the definition (8.43) of the function $G$. Then it is easily seen that $G$ is piecewise differentiable, apart from at the points $\sigma$ that are given by $q_{1} \sigma^{-2} / k_{1}=a \varrho^{2}+k q$. At these points $G$ is discontinuous, but is continuous to the left and to the right of such points. An integration by parts therefore yields

$$
\begin{aligned}
\sum_{Y_{1} / T^{1 / 2}<\xi_{1} \leqslant Y_{1}} \varpi\left(\xi_{1}\right) G\left(\xi_{1} / Y_{1}\right)= & \int_{Y_{1} / T^{1 / 2}}^{Y_{1}} G\left(t / Y_{1}\right) \mathrm{d} S(t) \\
= & S\left(Y_{1}\right) G(1)-S\left(Y_{1} / T^{1 / 2}\right) G\left(1 / T^{1 / 2}\right) \\
& -\frac{1}{Y_{1}} \int_{Y_{1} / T^{1 / 2}}^{Y_{1}} G^{\prime}\left(t / Y_{1}\right) S(t) \mathrm{d} t \\
& -\int_{1 / T^{1 / 2}}^{1} \sum_{\sigma}(G(\sigma+)-G(\sigma-)) S\left(Y_{1} t\right) \delta_{\sigma}(t),
\end{aligned}
$$

where the sum in the final term is over the discontinuities $\sigma$ of $G$, and $\delta_{\sigma}$ denotes the Dirac measure for $\sigma$.

We begin by estimating the contribution to (8.46) from the principal term $S_{P}$ in the estimate for $S$. This gives

$$
\int_{Y_{1} / T^{1 / 2}}^{Y_{1}} G\left(t / Y_{1}\right) \mathrm{d} S_{P}(t)=\frac{6}{\pi^{2}} Y_{1} \phi^{\prime}\left(k_{\ell} \xi_{2} \xi_{3} \xi_{\ell} \xi_{4} \xi_{5}\right) \int_{1 / T^{1 / 2}}^{1} G(w) \mathrm{d} w .
$$

The overall contribution in (8.42) from $S_{P}$ is therefore

$$
=B \frac{-6}{\pi^{2}} \sum_{\substack{k_{\ell} \mid \xi_{4} \xi_{5} \xi_{6} \\
\operatorname{gcd}\left(k_{\ell}, \xi_{2} \xi_{3}\right)=1}} \frac{\mu\left(k_{\ell}\right) \phi^{\prime}\left(k_{\ell} \xi_{2} \xi_{3} \xi_{\ell} \xi_{4} \xi_{5}\right)}{k_{\ell} \xi^{(0,2,2,3,3,3,3)}} \sum_{\begin{array}{c}
k_{1} \mid \xi_{2} \xi_{3} \xi_{6} \\
\operatorname{gcd}\left(k_{1}, q\right)=1
\end{array}} \mu\left(k_{1}\right) \int_{1 / T^{1 / 2}}^{1} G(w) \mathrm{d} w
$$

$$
=B u(\boldsymbol{\xi})+\mathrm{O}_{\varepsilon}\left(B / T^{1 / 2-\varepsilon}\right),
$$

where we have written

$$
u(\boldsymbol{\xi}):=\frac{-6}{\pi^{2}} \sum_{\substack{k_{\ell} \mid \xi_{4} \xi_{5} \xi_{6} \\
\operatorname{gcd}\left(k_{\ell}, \xi_{2} \xi_{3}\right)=1}} \frac{\mu\left(k_{\ell}\right) \phi^{\prime}\left(k_{\ell} \xi_{2} \xi_{3} \xi_{\ell} \xi_{4} \xi_{5}\right)}{k_{\ell} \xi^{(0,2,2,3,3,3,3)}} \sum_{\begin{array}{c}
k_{1} \mid \xi_{2} \xi_{3} \xi_{6} \\
\operatorname{gcd}\left(k_{1}, q\right)=1
\end{array}} \mu\left(k_{1}\right) \int_{0}^{1} G(w) \mathrm{d} w .
$$

Here we recall that the function $G$, as given by (8.43), depends intimately upon the values of $k_{1}$ and $k_{\ell}$. The quantity $u(\boldsymbol{\xi})$ is the general term of an absolutely convergent series which is completely independent of $B$. In fact, by using (8.44) it is easy to establish the upper bound

$$
u(\boldsymbol{\xi}) \ll_{\varepsilon} 2^{\omega\left(\xi_{2} \xi_{3} \xi_{6}\right)+\omega\left(\xi_{4} \xi_{5} \xi_{6}\right)} \frac{\left(\xi_{\ell}^{3} \xi_{4}^{2} \xi_{5}\right)^{1 / 2+\varepsilon}}{\xi^{(0,2,2,3,3,3,3)}} \ll_{\varepsilon} \frac{1}{q_{2^{1 / 2-3 \varepsilon}} \xi^{(0,1,1,1,1,1,1)}},
$$


whence

$$
\sum_{\substack{\xi_{2}, \ldots, \xi_{6} \in \mathbb{N} \\ q_{2}>T}}|u(\boldsymbol{\xi})| \ll_{\varepsilon} \frac{1}{T^{1 / 2-4 \varepsilon}} .
$$

On summing (8.48) over all relevant values of $\xi_{2}, \ldots, \xi_{6}$ such that $q_{2} \leqslant T$, we therefore obtain the overall contribution

$$
=\beta B+\mathrm{O}\left(\frac{B}{T^{1 / 2-4 \varepsilon}}\right)
$$

to (8.42) from $S_{P}$, where

$$
\beta:=\sum_{\substack{\xi_{2}, \ldots, \xi_{6} \in \mathbb{N} \\ \operatorname{gcd}\left(\xi_{\ell}, \xi_{2} \xi_{3}\right)=1}}\left|\mu\left(\xi_{2} \xi_{3} \xi_{4} \xi_{5}\right)\right| u(\boldsymbol{\xi}) .
$$

We proceed to estimate the overall contribution to the second line in (8.46) from the residual term $S_{R}$ in Lemma 24 . We shall need the observation that

$$
\int_{1 / T^{1 / 2}}^{1}\left|G^{\prime}(\alpha)\right| \mathrm{d} \alpha \ll_{\varepsilon} q^{1 / 2+\varepsilon} T^{1 / 2}+q_{1} T^{1 / 3}
$$

that follows easily from Lemma 23. Using this bound therefore yields the overall contribution

$$
\ll_{\varepsilon} Y_{1}^{\varepsilon} 2^{\omega\left(\xi_{2} \xi_{3} \xi_{6}\right)+\omega\left(\xi_{4} \xi_{5} \xi_{6}\right)}\left(q^{1 / 2+\varepsilon} T^{1 / 2}+q_{1} T^{1 / 3}\right) \ll_{\varepsilon} B^{\varepsilon}\left(q^{1 / 2} T^{1 / 2}+q_{1} T^{1 / 3}\right),
$$

to the second line. Given that $q_{2} \leqslant T$, the contribution in (8.42) is therefore

$$
\ll_{\varepsilon} \frac{B^{1 / 2+\varepsilon}}{\xi^{(0,1 / 2,0,3 / 2,1,1 / 2,0)}}\left(\left(\xi_{\ell}^{3} \xi_{4}^{2} \xi_{5}\right)^{1 / 2} T^{1 / 2} \frac{T}{q_{2}}+T^{11 / 6} \frac{q_{1}}{q_{2}{ }^{3 / 2}}\right) \ll_{\varepsilon} \frac{B^{1 / 2+\varepsilon} T^{11 / 6}}{\xi^{(0,5 / 2,2,1,2,3,4)}},
$$

whence we obtain a total contribution of

$$
\mathrm{O}\left(B^{1 / 2+2 \varepsilon} T^{11 / 6}\right) .
$$

It remains to examine the influence of the discontinuities of $G$ upon the final result. In other words, we must now estimate the overall contribution from the final line (8.46) when $S$ is replaced by $S_{R}$. Now we have

$$
\sum_{\sigma}(G(\sigma+)-G(\sigma-)) \delta_{\sigma}(\alpha)=\sum_{\substack{1 \leqslant \varrho \leqslant q \\ \operatorname{gcd}(\varrho, q)=1}} \delta_{-a \varrho^{2}+q \mathbb{Z}}\left(q_{1} / \alpha^{2} k_{1}\right) g_{2}\left(g_{1}(\alpha), \alpha\right) .
$$

Let $\alpha_{0}=1 / 2^{1 / 2}$. Then in view of (5.8) we have $g_{2}\left(g_{1}(\alpha), \alpha\right)=0$ for any $\alpha \leqslant \alpha_{0}$, and so for each $k_{1}$ it suffices to consider the values of $q_{1} / \alpha^{2} k_{1}$ in $-a \varrho^{2}+q \mathbb{Z}$ such that $\alpha>\alpha_{0}$. Hence

$$
\sum_{\sigma}(G(\sigma+)-G(\sigma-)) \delta_{\sigma}(\alpha)=\sum_{\substack{m \leqslant q_{1} / \alpha_{0}^{2} k_{1} \\ \operatorname{gcd}(m, q)=1}} \sum_{\substack{1 \leqslant \varrho \leqslant q \\-a \varrho^{2} \equiv m(\bmod q)}} \delta_{m}\left(q_{1} / \alpha^{2} k_{1}\right) g_{2}\left(g_{1}(\alpha), \alpha\right) .
$$

$4^{e}$ SÉRIE - TOME $40-2007-\mathrm{N}^{\circ} 1$ 
Now it is clear that there are at most $2^{\omega(q)}$ solutions $\varrho$ to the congruence $-a \varrho^{2} \equiv m(\bmod q)$, whence

$$
\int_{1 / T^{1 / 2}}^{1} \sum_{\sigma}(G(\sigma+)-G(\sigma-)) S_{R}\left(Y_{1} t\right) \delta_{\sigma}(t) \ll_{\varepsilon} q_{1} B^{\varepsilon} .
$$

This therefore yields a contribution of

$$
\ll_{\varepsilon} \frac{B^{1 / 2+\varepsilon} q_{1} T^{3 / 2}}{\xi^{(0,1 / 2,0,3 / 2,1,1 / 2,0)} q_{2}{ }^{3 / 2}} \ll_{\varepsilon} \frac{B^{1 / 2+\varepsilon} T^{3 / 2}}{\xi^{(0,5 / 2,2,1,2,3,4)}}
$$

in (8.42), since $q_{2} \leqslant T$. Thus the total contribution is

$$
\mathrm{O}_{\varepsilon}\left(B^{1 / 2+2 \varepsilon} T^{3 / 2}\right) .
$$

On combining the different contributions that we have estimated in (8.49), (8.51) and (8.52), and then combining this with Lemma 22, we have therefore established the estimate

$$
\sum_{\boldsymbol{\xi} \in \mathcal{A}(B, 1)} E_{2}(\boldsymbol{\xi})=\beta B+\mathrm{O}_{\varepsilon}\left(\frac{B}{T^{1 / 2-4 \varepsilon}}+B^{1 / 2+2 \varepsilon} T^{11 / 6}\right),
$$

for any $T \geqslant 1$. Making the choice $T=B^{3 / 14}$ therefore allows us to complete the proof of Proposition 2, on redefining the choice of $\varepsilon$.

\subsection{Summation over $\xi$}

The aim of this short section is to sum the main term in Lemma 21's estimate for $N^{\prime}(\boldsymbol{\xi})$ over all $\boldsymbol{\xi} \in \mathcal{F}$ such that the height condition (7.14) holds. Note that the definition (8.34) of $\vartheta$ ensures that this main term is zero unless $\boldsymbol{\xi} \in \mathcal{F}$.

Define the arithmetic function

$$
\Delta(n)=B^{-5 / 6} \sum_{\substack{\boldsymbol{\xi} \in \mathbb{N}^{7} \\ \xi^{(2,3,4,3,5,6)}=n}} \frac{\vartheta(\boldsymbol{\xi}) X_{1} X_{2}}{\xi_{\ell}^{3} \xi_{4}^{2} \xi_{5}}
$$

for any $n \in \mathbb{N}$, where $X_{1}, X_{2}$ are given by (7.13). Let $\varepsilon>0$ and let $B \geqslant 1$ be such that $B \in \mathbb{R} \backslash \overline{\mathbb{Q}}$. Then on bringing together the estimates in Lemmas 16 and 21, in addition to the handling of the error terms in Propositions 1 and 2, we therefore deduce that

$$
\# \mathcal{E}(B)=B^{5 / 6} \sum_{n \leqslant B} \Delta(n) g_{3}\left((n / B)^{1 / 6}\right)+\beta B+\mathrm{O}_{\varepsilon}\left(B^{43 / 48+\varepsilon}\right)
$$

in Lemma 15. But we may now combine this with Lemmas 12 and 15 to deduce the following basic result.

Lemma 25. - Let $\varepsilon>0$ and let $B \geqslant 1$. Then we have

$$
N_{U, H}(B)=2 B^{5 / 6} \sum_{n \leqslant B} \Delta(n) g_{3}\left((n / B)^{1 / 6}\right)+\left(\frac{12}{\pi^{2}}+2 \beta\right) B+\mathrm{O}_{\varepsilon}\left(B^{43 / 48+\varepsilon}\right),
$$

where $g_{3}$ is given by (8.36), $\beta$ is given by (8.50) and $\Delta$ is given by (8.53). 
Proof. - The result is immediate if $B \in \mathbb{R} \backslash \overline{\mathbb{Q}}$. Let $B_{1}, B_{2}, B_{3}, \ldots$ be an arbitrary sequence of transcendental numbers such that $\left\lfloor B_{k}\right\rfloor=\lfloor B\rfloor$ for each $k \in \mathbb{N}$ and $\lim _{k \rightarrow \infty} B_{k}=B$. Then for each $k \in \mathbb{N}$ we have

$$
\begin{aligned}
N_{U, H}(B) & =N_{U, H}\left(B_{k}\right) \\
& =2 B^{5 / 6} \sum_{n \leqslant B} \Delta(n) g_{3}\left(\left(n / B_{k}\right)^{1 / 6}\right)+\left(\frac{12}{\pi^{2}}+2 \beta\right) B+\mathrm{O}_{\varepsilon}\left(B^{43 / 48+\varepsilon}\right) .
\end{aligned}
$$

Taking the limit as $k \rightarrow \infty$ therefore yields the statement of Lemma 25 .

\section{Proof of Theorem 1: dénouement}

In this section we complete the proof of Theorem 1. Our main task is to estimate the quantity

$$
M(x):=\sum_{n \leqslant x} \Delta(n),
$$

for any $x \geqslant 1$. This will be achieved in Lemma 27 below. First it will be necessary to examine the analytic properties of the corresponding Dirichlet series $F(s):=\sum_{n=1}^{\infty} \Delta(n) n^{-s}$. On recalling the definition (8.53) of $\Delta$, and those of $X_{1}, X_{2}$, we see that

$$
\Delta(n)=\sum_{\substack{\boldsymbol{\xi} \in \mathbb{N}^{7} \\ \xi^{(2,3,4,3,4,5,6)}=n}} \frac{\vartheta(\boldsymbol{\xi}) n^{1 / 6}}{\xi^{(1,1,1,1,1,1,1)}}
$$

in (8.53). Hence

$$
F(s-5 / 6)=\sum_{\boldsymbol{\xi} \in \mathbb{N}^{7}} \frac{\vartheta(\boldsymbol{\xi}) \xi^{(2,3,4,3,4,5,6)}}{\xi_{1}^{2 s+1} \xi_{2}^{3 s+1} \xi_{3}^{4 s+1} \xi_{\ell}^{3 s+1} \xi_{4}^{4 s+1} \xi_{5}^{5 s+1} \xi_{6}^{6 s+1},}
$$

where $\vartheta(\boldsymbol{\xi})$ is given by (8.34). Now recall the definitions (1.5), (1.6) of $E_{1}(s)$ and $E_{2}(s)$, and define the half-plane

$$
\mathcal{H}_{\theta}:=\{s \in \mathbb{C}: \Re e(s)>\theta\}
$$

for any $\theta>0$. We proceed by establishing the following result.

LEMmA 26. - Let $\varepsilon>0$. Then there exists a function $G_{1,1}(s)$ that is holomorphic and bounded on $\mathcal{H}_{5 / 6+\varepsilon}$, such that

$$
F(s-5 / 6)=E_{1}(s) E_{2}(s) G_{1,1}(s) .
$$

Proof. - We have already seen in (9.1) an explicit formula for $F(s)$. On writing $F(s+1 / 6)=$ $\prod_{p} F_{p}(s+1 / 6)$ as a product of local factors, a straightforward calculation reveals that

$$
\begin{aligned}
F_{p}(s+1 / 6)= & +\frac{(1-1 / p)^{2}}{\left(p^{6 s+1}-1\right)}\left(\frac{p^{2 s+1}}{p^{2 s+1}-1}+\frac{p^{2 s+1} p^{6 s+1}}{p^{4 s+1}\left(p^{2 s+1}-1\right)}\right. \\
& +\frac{p^{6 s+1}}{(1-1 / p) p^{3 s+1}}+\frac{1}{p^{3 s+1}-1}+\frac{p^{3 s+1} p^{6 s+1}}{p^{4 s+1}\left(p^{3 s+1}-1\right)} \\
& \left.+\frac{p^{3 s+1} p^{6 s+1}}{p^{5 s+1}\left(p^{3 s+1}-1\right)}\right)+\frac{1-1 / p}{p^{2 s+1}-1}+\frac{1-1 / p}{p^{3 s+1}-1}
\end{aligned}
$$

$4^{\mathrm{e}}$ SÉRIE - TOME $40-2007-\mathrm{N}^{\circ} 1$ 
We proceed to calculate $F_{p}(s+1 / 6)\left(1-1 / p^{6 s+1}\right)\left(1-1 / p^{2 s+1}\right)\left(1-1 / p^{3 s+1}\right)$ on $\mathcal{H}_{-1 / 6+\varepsilon}$. Thus we have

$$
\begin{aligned}
& F_{p}(s+1 / 6)\left(1-1 / p^{6 s+1}\right)\left(1-1 / p^{2 s+1}\right)\left(1-1 / p^{3 s+1}\right) \\
& \quad=1+\frac{1}{p^{3 s+1}}+\frac{2}{p^{4 s+1}}+\frac{1}{p^{5 s+1}}-\frac{2}{p^{7 s+2}}+\mathrm{O}_{\varepsilon}\left(\frac{1}{p^{1+\varepsilon}}\right)
\end{aligned}
$$

whence

$$
\begin{aligned}
\frac{F_{p}(s+1 / 6)}{E_{1, p}(s+1)} & =1-\frac{1}{p^{10 s+2}}-\frac{2}{p^{9 s+2}}-\frac{4}{p^{8 s+2}}-\frac{4}{p^{7 s+2}}+\frac{2}{p^{14 s+3}}+\frac{5}{p^{13 s+3}}+\mathrm{O}_{\varepsilon}\left(\frac{1}{p^{1+\varepsilon}}\right) \\
& =E_{2, p}(s+1)\left(1+\mathrm{O}_{\varepsilon}\left(\frac{1}{p^{1+\varepsilon}}\right)\right) .
\end{aligned}
$$

This therefore establishes the lemma.

Let $\varepsilon>0$ and recall the properties of $E_{1}(s), E_{2}(s)$ that were outlined in $\S 1$. In particular we clearly have

$$
E_{1}(s+1)=\prod_{i \in\{1,2,3, \ell, 4,5,6\}} \zeta\left(\lambda_{i} s+1\right),
$$

where $\boldsymbol{\lambda}=\left(\lambda_{1}, \ldots, \lambda_{6}\right)$ is given by $(2.1)$, and so

$$
E_{1}(s)=\frac{1}{\prod_{i} \lambda_{i}}(s-1)^{-7}+\mathrm{O}\left((s-1)^{-6}\right)
$$

as $s \rightarrow 1$. It follows from Lemma 26 that $F(s-5 / 6)$ is meromorphic on $\mathcal{H}_{9 / 10+\varepsilon}$, with a pole of order 7 at $s=1$. Moreover, the function

$$
G(s):=\frac{F(s-5 / 6)}{E_{1}(s)}
$$

is holomorphic and bounded on $\mathcal{H}_{9 / 10+\varepsilon}$. Now a simple calculation reveals that for any $x \geqslant 1$ we have

$$
\operatorname{Res}_{s=1}\left(\frac{F(s-5 / 6) x^{s-5 / 6}}{s-5 / 6}\right)=\frac{6 G(1) x^{1 / 6} Q_{0}(\log x)}{6 ! \cdot \prod_{i} \lambda_{i}},
$$

for some monic polynomial $Q_{0}$ of degree 6 , and where

$$
G(1)=\prod_{p}\left(1-\frac{1}{p}\right)^{7}\left(1+\frac{7}{p}+\frac{1}{p^{2}}\right) .
$$

We are now ready to establish the following estimate for $M(x)$.

Lemma 27. - Let $\varepsilon>0$. Then there exists a monic polynomial $Q_{1}$ of degree 6 such that

$$
M(x)=\frac{G(1) x^{1 / 6} Q_{1}(\log x)}{1036800}+\mathrm{O}_{\varepsilon}\left(x^{1 / 6-1 / 11+\varepsilon}\right),
$$

for any $x \geqslant 1$. 
Proof. - Our starting point is the pair of inequalities

$$
\frac{1}{y} \int_{x-y}^{x} M(t) \mathrm{d} t \leqslant M(x) \leqslant \frac{1}{y} \int_{x}^{x+y} M(t) \mathrm{d} t,
$$

that are valid for any $1 \leqslant y<x$. Note that $M(x)$ is an increasing function. This approach to estimating $M(x)$ is a variant of Perron's formula which has the advantage of giving rise to absolutely convergent complex integrals. We shall only estimate the right-hand side of (9.5), since the integral on the left ultimately yields precisely the same estimate, as is easily checked. An application of Perron's formula yields

$$
\begin{aligned}
\frac{1}{y} \int_{x}^{x+y} M(t) \mathrm{d} t & =\frac{1}{2 \pi i} \int_{1 / 6+1 / \log x-i \infty}^{1 / 6+1 / \log x+i \infty} F(s) \frac{(x+y)^{s+1}-x^{s+1}}{y s(s+1)} \mathrm{d} s \\
& =\frac{1}{2 \pi i} \int_{\kappa-i \infty}^{\kappa+i \infty} F(s-5 / 6) \frac{(x+y)^{s+1 / 6}-x^{s+1 / 6}}{y(s-5 / 6)(s+1 / 6)} \mathrm{d} s
\end{aligned}
$$

with $\kappa=1+1 / \log x$. Let $\delta=1 / 10+\varepsilon$. Then we shall apply Cauchy's residue theorem to the rectangular contour $\mathcal{C}$ joining the points

$$
\kappa-i T, \quad \kappa+i T, \quad 1-\delta+i T, \quad 1-\delta-i T,
$$

for some value of $T \in[1, x]$ to be selected in due course.

We shall employ the well-known convexity bounds

$$
\zeta(\sigma+i \tau) \ll_{\varepsilon} \begin{cases}|\tau|^{(3-4 \sigma) / 6+\varepsilon}, & \text { if } \sigma \in[0,1 / 2] \\ |\tau|^{(1-\sigma) / 3+\varepsilon}, & \text { if } \sigma \in[1 / 2,1]\end{cases}
$$

valid for any $|\tau| \geqslant 1$ (see Tenenbaum [17, §II.3.4], for example). It follows from these that

$$
F(\sigma-5 / 6+i \tau) \ll E_{1}(\sigma+i \tau) \ll_{\varepsilon}(1+|\tau|)^{\mu_{F}(\sigma)+\varepsilon}
$$

on $\mathcal{C}$, with

$$
\mu_{F}(\sigma) \leqslant \begin{cases}\max \{9(1-\sigma), 0\}, & \text { if } \sigma \geqslant 11 / 12 \\ 65 / 6-11 \sigma, & \text { if } 9 / 10 \leqslant \sigma \leqslant 11 / 12\end{cases}
$$

Moreover, we have

$$
(x+y)^{s+1 / 6}-x^{s+1 / 6} \ll x^{\sigma+1 / 6}(y(1+|\tau|) / x)^{\alpha},
$$

for any $\alpha \in[0,1]$. On taking $\alpha=1$ here we may therefore estimate the contribution from the horizontal strips as being

$$
\begin{aligned}
\int_{1-\delta \pm i T}^{\kappa \pm i T}\left|F(s-5 / 6) \frac{(x+y)^{s+1 / 6}-x^{s+1 / 6}}{y(s-5 / 6)(s+1 / 6)}\right| \mathrm{d} s & \ll_{\varepsilon} \int_{1-\delta}^{\kappa} x^{\sigma-5 / 6} T^{\mu_{F}(\sigma)-1+\varepsilon} \mathrm{d} \sigma \\
& \ll_{\varepsilon} \frac{x^{1 / 6+\varepsilon}}{T}+x^{1 / 6-\delta+\varepsilon},
\end{aligned}
$$

$4^{\mathrm{e}}$ SÉRIE - TOME $40-2007-\mathrm{N}^{\circ} 1$ 
since $T \geqslant 1$. In a similar fashion we see that

$$
\int_{\kappa \pm i T}^{\kappa \pm i \infty}\left|F(s-5 / 6) \frac{(x+y)^{s+1 / 6}-x^{s+1 / 6}}{y(s-5 / 6)(s+1 / 6)}\right| \mathrm{d} s \ll_{\varepsilon} \frac{x^{1 / 6+\varepsilon}}{T} .
$$

On applying (9.6) with $\alpha=3 / 2-6 \delta$, we see that the contribution from the line $\Re e(s)=1-\delta$ is

$$
\begin{aligned}
& \ll \int_{1-\delta-i T}^{1-\delta+i T}\left|F(s-5 / 6) \frac{(x+y)^{s+1 / 6}-x^{s+1 / 6}}{y(s-5 / 6)(s+1 / 6)}\right| \mathrm{d} s \\
& \ll x^{5 \delta-1 / 3} y^{1 / 2-6 \delta} \int_{-T}^{T}\left|\frac{E_{1}(1-\delta+i \tau)}{(1+|\tau|)^{1 / 2+6 \delta}}\right| \mathrm{d} \tau \\
& \ll x^{5 \delta-1 / 3} y^{1 / 2-6 \delta} \log T \sup _{1 \leqslant U \leqslant T} \frac{1}{U^{1 / 2+6 \delta}} \int_{0}^{U}\left|E_{1}(1-\delta+i \tau)\right| \mathrm{d} \tau .
\end{aligned}
$$

Now let

$$
J_{k}(\sigma, U):=\left(\frac{1}{U} \int_{0}^{U}|\zeta(1-\sigma+i \tau)|^{k} \mathrm{~d} \tau\right)^{1 / k} .
$$

Then an application of Hölder's inequality, with weights (10,5,10/3,4,20/3), yields

$$
\begin{aligned}
\frac{1}{U} \int_{0}^{U}\left|E_{1}(1-\delta+i \tau)\right| \mathrm{d} \tau \ll & J_{10}(2 \delta, 2 U) J_{10}(3 \delta, 3 U)^{2} J_{20 / 3}(4 \delta, 4 U)^{2} \\
& \times J_{4}(5 \delta, 5 U) J_{20 / 3}(6 \delta, 6 U) .
\end{aligned}
$$

It therefore follows from classical upper bounds for the fractional moment of the Riemann zeta function (see Ivić [13, Theorem 2.4], for example) that

$$
\begin{aligned}
& J_{10}(2 \delta, 2 U) \ll_{\varepsilon} U^{\varepsilon}, \quad J_{10}(3 \delta, 3 U) \ll_{\varepsilon} U^{\varepsilon}, \quad J_{20 / 3}(4 \delta, 4 U) \ll_{\varepsilon} U^{\varepsilon}, \\
& J_{4}(5 \delta, 5 U) \ll_{\varepsilon} U^{\varepsilon}, \quad J_{20 / 3}(6 \delta, 6 U) \ll_{\varepsilon} U^{6 \delta-1 / 2},
\end{aligned}
$$

that are valid when $\varepsilon$ is chosen to be sufficiently small. To obtain the final estimate here we have used the functional equation of the Riemann zeta function in the form $|\zeta(1-6 \delta+i \tau)| \asymp$ $|\tau|^{6 \delta-1 / 2}|\zeta(6 \delta+i \tau)|$, for any $|\tau| \geqslant 1$. Putting this together we therefore obtain the estimate

$$
\int_{1-\delta-i T}^{1-\delta+i T}\left|F(s-5 / 6) \frac{(x+y)^{s+1 / 6}-x^{s+1 / 6}}{y(s-5 / 6)(s+1 / 6)}\right| \mathrm{d} s \ll_{\varepsilon} x^{5 \delta-1 / 3} y^{1 / 2-6 \delta} T^{\varepsilon} .
$$

We may now collect together all of our various estimates, together with the choices $T=x$ and $y=x^{10 / 11}$, in order to deduce from (2.1), (9.3) and (9.5) that there exists a monic polynomial $Q_{1}$ of degree 6 such that

$$
\begin{aligned}
M(x) & =\operatorname{Res}_{s=1}\left(F(s-5 / 6) \frac{(x+y)^{s+1 / 6}-x^{s+1 / 6}}{y(s-5 / 6)(s+1 / 6)}\right)+\mathrm{O}_{\varepsilon}\left(x^{1 / 6-1 / 11+\varepsilon}\right) \\
& =\frac{6 G(1) x^{1 / 6} Q_{1}(\log x)}{6 ! \cdot \prod_{i} \lambda_{i}}+\mathrm{O}_{\varepsilon}\left(x^{1 / 6-1 / 11+\varepsilon}\right),
\end{aligned}
$$

for any $x \geqslant 1$. This completes the proof of Lemma 27, since $\prod_{i} \lambda_{i}=1036800$ by (2.1). 
We are now ready to complete the proof of Theorem 1 via an application of partial summation in Lemma 25. Thus it follows from Lemma 27 that there exists a monic polynomial $Q_{2}$ of degree 6 such that

$$
\begin{aligned}
\sum_{n \leqslant B} \Delta(n) g_{3}\left((n / B)^{1 / 6}\right)= & \frac{G(1)}{1036800} \int_{0}^{B} g_{3}\left((n / B)^{1 / 6}\right) \frac{\mathrm{d}}{\mathrm{d} u}\left(u^{1 / 6} Q_{1}(\log u)\right) \mathrm{d} u \\
& +\mathrm{O}_{\varepsilon}\left(B^{1 / 6-1 / 11+\varepsilon}\right) \\
= & \frac{G(1) B^{1 / 6} Q_{2}(\log B)}{1036800} \int_{0}^{1} g_{3}(v) \mathrm{d} v+\mathrm{O}_{\varepsilon}\left(B^{5 / 66+\varepsilon}\right)
\end{aligned}
$$

for any $B \geqslant 1$. On noting that

$$
\omega_{\infty}=12 \int_{0}^{1} g_{3}(v) \mathrm{d} v
$$

in (1.3), and recalling the equality (9.4) for $G(1)$, we may therefore insert this estimate into Lemma 25 in order to conclude the proof of Theorem 1.

\section{Proof of Theorem 2}

The proof of Theorem 2 is almost identical to the corresponding proof of [3, Theorem 1], and so we shall be very brief. Recall the definition (9.2) of the half-plane $\mathcal{H}_{\theta}$ for any $\theta>0$. Following $[3, \S 6]$ therefore, we easily deduce from Lemma 25 that

$$
Z_{U, H}(s):=\sum_{x \in U(\mathbb{Q})} \frac{1}{H(x)^{s}}=Z_{1}(s)+\frac{12 / \pi^{2}+2 \beta}{s-1}+G_{2}(s),
$$

for $s \in \mathcal{H}_{1}$, where

$$
G_{2}(s)=s \int_{1}^{\infty} t^{-s-1} R(t) \mathrm{d} t
$$

for some function $R(t)$ such that $R(t) \ll_{\varepsilon} t^{43 / 48+\varepsilon}$, and

$$
Z_{1}(s)=2 s \int_{1}^{\infty} t^{-s-1 / 6} \sum_{n \leqslant t} \Delta(n) g_{3}\left(\left(\frac{n}{t}\right)^{1 / 6}\right) \mathrm{d} t .
$$

Now it is clear that $G_{2}(s)$ is holomorphic on $\mathcal{H}_{43 / 48+\varepsilon}$, and satisfies $G_{2}(s) \ll 1+|\Im m(s)|$ on this domain. Moreover one readily deduces from (1.5), (1.6) and Lemma 26 that

$$
Z_{1}(s)=2 s F(s-5 / 6) \int_{1}^{\infty} t^{-s-1 / 6} g_{3}\left(1 / t^{1 / 6}\right) \mathrm{d} t=E_{1}(s) E_{2}(s) G_{1,1}(s) G_{1,2}(s)
$$

$4^{\text {e }}$ SÉRIE - TOME $40-2007-\mathrm{N}^{\circ} 1$ 
on $\mathcal{H}_{1}$. Here the function $G_{1,1}(s)$ is holomorphic and bounded on $\mathcal{H}_{5 / 6+\varepsilon}$, and

$$
G_{1,2}(s)=12 s \int_{0}^{1} v^{6 s-6} g_{3}(v) \mathrm{d} v .
$$

A simple calculation reveals that $G_{1,2}(1)=\omega_{\infty}$, in the notation of (1.3). Moreover, an application of partial integration yields

$$
G_{1,2}(s)=\frac{12 s}{6 s-5}\left(g_{3}(1)-\int_{0}^{1} v^{6 s-5} g_{3}{ }^{\prime}(v) \mathrm{d} v\right),
$$

whence $G_{1,2}(s)$ is holomorphic and bounded on $\mathcal{H}_{5 / 6+\varepsilon}$. In view of the fact that $G_{1,1}(1) G_{1,2}(1) \neq 0$, we may therefore set

$$
G_{1}(s):=G_{1,1}(s) G_{1,2}(s)
$$

on $\mathcal{H}_{5 / 6+\varepsilon}$, in order to complete the proof of Theorem 2 .

\section{Acknowledgements}

Part of this work was undertaken while the second author was attending the Diophantine Geometry intensive research period at the Centro di Ricerca Matematica Ennio De Giorgi in Pisa, and the third author was visiting Brendan Hassett at Rice University. The paper was finalised while the first author was at the École normale supérieure, and the second author was at Oxford University supported by EPSRC grant number GR/R93155/01. The hospitality and financial support of all these institutions is gratefully acknowledged. Finally, the authors would like to thank the anonymous referee for his extremely attentive reading of the manuscript and numerous helpful suggestions. In particular, the referee's comments have helped to simplify the proofs of Lemmas 2 and 4, and led to an overall improvement in Lemma 22.

\section{REFERENCES}

[1] BatyreV V.V., Tschinkel Y., Tamagawa numbers of polarized algebraic varieties, Astérisque 251 (1998) 299-340.

[2] DE LA BRETÈCHE R., Sur le nombre de points de hauteur bornée d'une certaine surface cubique singulière, Astérisque 251 (1998) 51-77.

[3] DE LA BRetèche R., Browning T.D., On Manin's conjecture for singular del Pezzo surfaces of degree four, I, Michigan Math. J., in press.

[4] De LA Bretèche R., BRowning T.D., On Manin's conjecture for singular del Pezzo surfaces of degree four, II, Math. Proc. Camb. Phil. Soc., in press.

[5] BRowning T.D., The density of rational points on a certain singular cubic surface, J. Number Theory 119 (2006) 242-283.

[6] BRuCE J.W., Wall C.T.C., On the classification of cubic surfaces, J. London Math. Soc. 19 (1979) 245-256.

[7] Chambert-Loir A., TsChinkel Y., On the distribution of points of bounded height on equivariant compactifications of vector groups, Invent. Math. 148 (2002) 421-452.

[8] Derenthal U., Manin's conjecture for a certain singular cubic surface, math.NT/0504016, 2005. 
[9] Franke J., Manin Y.I., Tschinkel Y., Rational points of bounded height on Fano varieties, Invent. Math. 95 (1989) 421-435.

[10] Hassett B., Tschinkel Y., Universal torsors and Cox rings, in: Arithmetic of Higher-Dimensional Algebraic Varieties, Palo Alto, CA, 2002, in: Progr. Math., vol. 226, Birkhäuser, Basel, 2004, pp. 149-173.

[11] Heath-Brown D.R., The density of rational points on cubic surfaces, Acta Arith. 79 (1997) 17-30.

[12] HEATH-BRown D.R., The density of rational points on Cayley's cubic surface, in: Proceedings of the Session in Analytic Number Theory and Diophantine Equations, in: Bonner Math. Schriften, vol. 360, Univ. Bonn, Bonn, 2003.

[13] IVIĆ A., The Riemann Zeta-Function, John Wiley \& Sons Inc., New York, 1985.

[14] Loxton J.H., Vaughan R.C., The estimation of complete exponential sums, Canad. Math. Bull. 28 (1985) 440-454.

[15] PeYre E., Hauteurs et nombres de Tamagawa sur les variétés de Fano, Duke Math. J. 79 (1995) $101-218$

[16] Salberger P., Tamagawa measures on universal torsors and points of bounded height on Fano varieties, Astérisque 251 (1998) 91-258.

[17] Tenenbaum G., Introduction to Analytic and Probabilistic Number Theory, translated from the second French ed., Cambridge Studies in Advanced Mathematics, vol. 46, Cambridge Univ. Press, Cambridge, UK, 1995.

[18] Titchmarsh E.C., The Theory of the Riemann Zeta-Function, second ed., revised by D.R. HeathBrown, Oxford Univ. Press, Oxford, UK, 1986.

[19] VaAler J.D., Some extremal functions in Fourier analysis, Bull. Amer. Math. Soc. 12 (1985) 183216.

[20] Vaughan R.C., The Hardy-Littlewood Method, second ed., Cambridge Tracts in Mathematics, vol. 125, Cambridge Univ. Press, Cambridge, UK, 1997.

[21] WeIL A., Sur les courbes algébriques et les variétés qui s'en déduisent, Pub. Inst. Math. Strasbourg 7 (1948) 1-85.

(Manuscrit reçu le 20 septembre 2005; accepté, après révision, le 5 décembre 2006.)

\footnotetext{
Régis DE LA BRETÈCHE UMR 7586, Case 7012, Université Paris 7 - Denis Diderot, 2, place Jussieu, F-75251 Paris cedex 05, France E-mail: breteche@math.jussieu.fr
}

Tim D. BRownING School of Mathematics, University of Bristol, Bristol BS8 1TW, UK E-mail: t.d.browning@bristol.ac.uk

Ulrich DERENTHAL Institut für Mathematik, Universität Zürich, Winterthurerstrasse 190, 8057 Zürich, Switzerland

E-mail: ulrich.derenthal@math.unizh.ch

$4^{\mathrm{e}}$ SÉRIE - TOME $40-2007-\mathrm{N}^{\circ} 1$ 